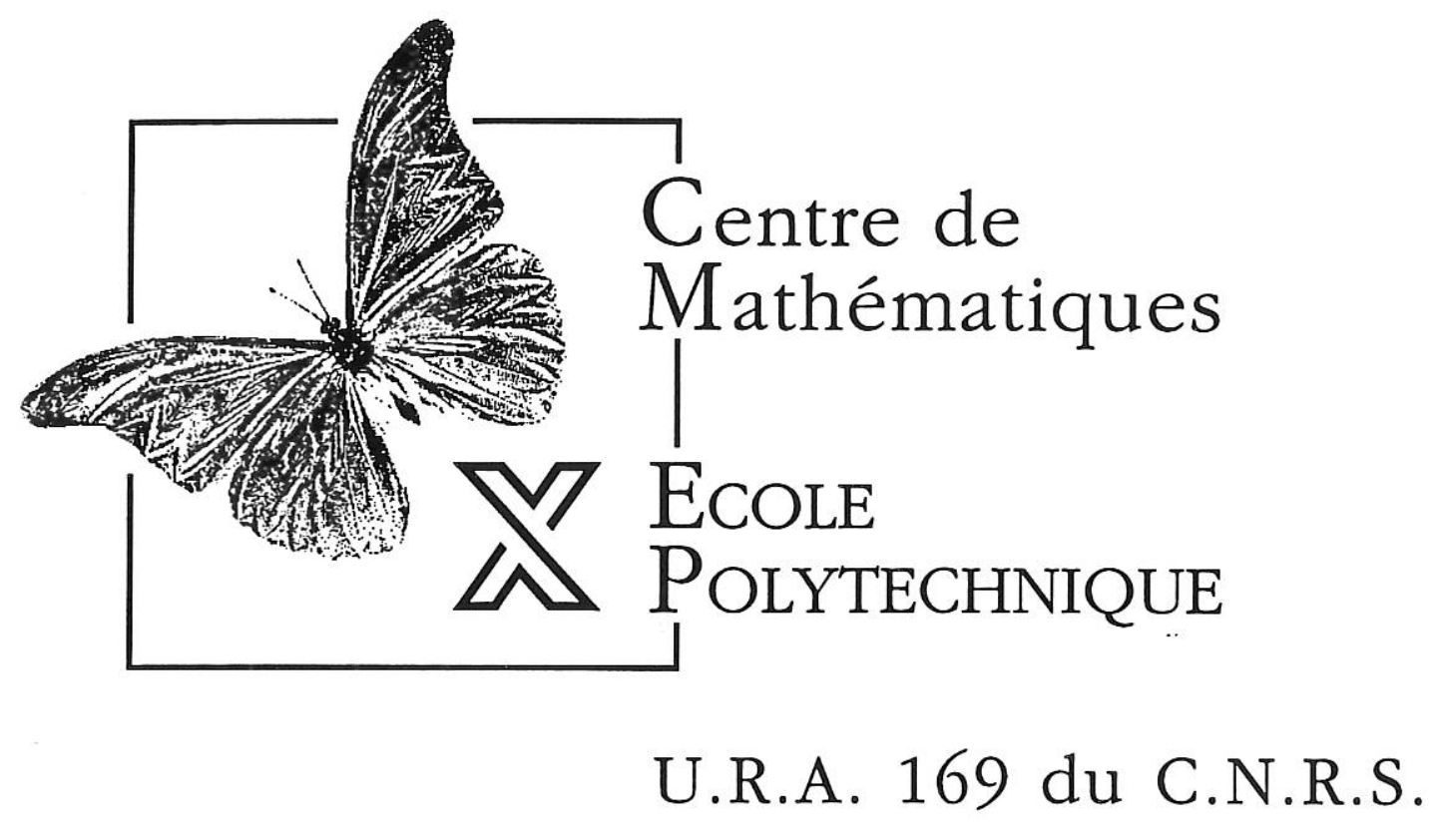

Boundary effect for an elliptic Neumann problem with critical nonlinearity

\title{
Olivier REY
}

\section{F-91128 Palaiseau Cedex}

Tél. : ((33)) (1) 69334088 • Fax : ((33)) (1) 69333019

Internet : secret@orphee.polytechnique.fr 
RÉSUMÉ. - Nous étudions les problèmes elliptiques avec non-linéarité critique et conditions au bord de Neumann de la forme $\left(P_{\mu}\right):-\Delta u+\mu u=$ $u^{\frac{n+2}{n-2}}, u>0$ sur $\Omega ; \frac{\partial u}{\partial \nu}=0$ sur $\partial \Omega$ - où $\Omega$ est un domaine borné et régulier de $\mathbf{R}^{n}, n \geq 3$, et $\mu$ est un paramètre strictement positif. Nous montrons, pour $n \geq 7$, que si $u$ est une solution de $\left(P_{\mu}\right)$ d'énergie petite, $u$ se concentre quand $\mu$ tend vers l'infini en un point du bord où la courbure moyenne $H$ est positive, et critique quand elle est strictement positive. Réciproquement, nous montrons, pour $n \geq 5$, que si $a>0$ est une valeur critique de $H$ induisant une différence de topologie entre les ensembles de niveau de cette fonction, il existe pour $\mu$ assez grand une solution de $\left(P_{\mu}\right)$ qui se concentre en un point $y$ du bord tel que $H(y)=a$ et $H^{\prime}(y)=0$. Enfin, si $n \geq 6$ et $y^{1}, \cdots, y^{k}$ sont $k$ points critiques distincts et non-dégénérés de $H$, il existe pour $\mu$ assez grand une solution de $\left(P_{\mu}\right)$ qui se concentre en chacun des points $y^{i}, 1 \leq i \leq k$.

Abstract. - We are interested in elliptic problems with critical nonlinearity and Neumann boundary conditions, namely $\left(P_{\mu}\right):-\Delta u+\mu u=$ $u^{\frac{n+2}{n-2}}, u>0$ in $\Omega ; \frac{\partial u}{\partial \nu}=0$ on $\partial \Omega$ - where $\Omega$ is a smooth bounded domain in $\mathbf{R}^{n}, n \geq 3$, and $\mu$ is a strictly positive parameter. We show, for $n \geq 7$, and $u$ a small energy solution of $\left(P_{\mu}\right)$, that $u$ concentrates as $\mu$ goes to infinity at a point of the boundary such that the mean curvature $H$ is positive, and critical if it is strictly positive. Conversely, we show, for $n \geq 5$, and $a>0$ a critical value for $H$ inducing a difference of topology between the level sets of $H$, that there exists for $\mu$ large enough a solution of $\left(P_{\mu}\right)$ which concentrates at a point $y$ of the boundary such that $H(y)=a$ and $H^{\prime}(y)=0$. Lastly, if $n \geq 6$ and $y^{1}, \cdots, y^{k}$ are $k$ distinct critical points of $H$, there exists for $\mu$ large enough a solution of $\left(P_{\mu}\right)$ which concentrates at each of the points $y^{i}$, $1 \leq i \leq k$.

Classification AMS. - $\quad 35$ J 65

MotS-CLÉS. - problèmes variationnels non-compacts, problèmes elliptiques avec non-linéarité critique, conditions au bord de Neumann, courbure moyenne.

KEYWORDS. - noncompact variational problems, elliptic problems with critical nonlinearity, Neumann boundary conditions, mean curvature. 


\section{1 - INTRODUCTION AND RESULTS}

This paper is devoted to the study of elliptic problems with critical nonlinearity and Neumann boundary conditions, namely

$$
\left\{\begin{aligned}
-\Delta u+\mu u & =u^{p} & & \text { in } \Omega \\
u & >0 & & \text { in } \Omega \\
\frac{\partial u}{\partial \nu} & =0 & & \text { on } \partial \Omega
\end{aligned}\right.
$$

where $\mu$ is a strictly positive constant, $\Omega$ is a smooth and bounded domain in $\mathbb{R}^{n}, n \geq$ 3 , and $p=\frac{n+2}{n-2}$.

Such kind of problems appears in the modelization of activator-inhibitor systems [16], as chemotactic behaviour of cells [12]. If $n=1,2$, or $n \geq 3$ and $1<p<\frac{n+2}{n-2}$, standard variational methods apply. In particular, Lin, $\mathrm{Ni}$ and Takagi [14], $\mathrm{Li}$ and $\mathrm{Ni}$ [13] proved that (1.1) does not have any nonconstant solution for small $\mu$, whereas it admits at least one for $\mu$ sufficiently large. It was conjectured that this situation was actually general : in contrast with the similar Dirichlet problem, in which the exponent $p$ plays a crucial role, $\mu$ would have been here the most important parameter to decide of existence or nonexistence of nontrivial solutions. However, by proving that for $n=4,5,6, p=\frac{n+2}{n-2}$ and $\Omega$ a ball, the problem did admit a nontrivial solution for any $\mu$ strictly positive, Adimurthi and Yadava [2] - see also Budd, Knaap and Peletier [10] - showed that the feature in the critical case is different, independently of the arguments which have anyhow to be modifed with respect to the subcritical case.

Adimurthi and Mancini [1] - see also Wang [20] - established that for any $\Omega$ in $\mathbb{R}^{n}, n \geq 3$, and $p=\frac{n+2}{n-2}$, (1.1) admits a solution for $\mu$ large enough. They use the same method as Brezis and Nirenberg in [9], which consists in proving, by the choice of suitable test functions, that the infinimum of the functional associated to the problem is below the first level at which a lack of compactness occurs. Any minimizing sequence thus converges to a nontrivial solution. The considered test functions are solutions to the equation

$$
-\Delta u=u^{\frac{n+2}{n-2}}, u>0 \text { in } \mathbb{R}^{n}
$$

which, with the additional assumptions $u \in L^{\frac{2 n}{n-2}}\left(\mathbb{R}^{n}\right), \nabla u \in L^{2}\left(\mathbb{R}^{n}\right)$, may all be written as

$$
U_{\lambda, y}(x)=\frac{\lambda^{\frac{n-2}{2}}}{\left(1+\lambda^{2}|x-y|^{2}\right)^{\frac{n-2}{2}}} \quad \lambda>0, y \in \mathbb{R}^{n}
$$


up to the multiplicative constant

$$
\bar{\alpha}=(n(n-2))^{\frac{n-2}{4}} .
$$

Setting

$$
I_{\mu}(u)=\frac{\int_{\Omega}|\nabla u|^{2}+\mu \int_{\Omega} u^{2}}{\left(\int_{\Omega}|u|^{p+1}\right)^{\frac{2}{p+1}}} \quad u \in H^{1}(\Omega), p=\frac{n+2}{n-2}
$$

a functional whose positive critical points, up to a multiplicative constant, are solutions to (1.1), and denoting

$$
S=\inf _{\substack{u \in H_{0}^{1}(\Omega) \\ u \neq 0}} \frac{\int_{\Omega}|\nabla u|^{2}}{\left(\int_{\Omega}|u|^{p+1}\right)^{\frac{2}{p+1}}} \quad p=\frac{n+2}{n-2}
$$

the best Sobolev constant, it follows from a standard application of the concentration - compactness principle [15] that $I_{\mu}$ is compact below the level $S / 2^{2 / n}$. On the other hand, expanding $I_{\mu}\left(U_{\lambda, y}\right)$ as $y \in \partial \Omega, H(y)>0$, where $H$ denotes the mean curvature of $\partial \Omega$, and $\lambda$ going to infinity, one obtains [1, Lemma 2.2]

$$
\inf _{u \in H_{0}^{1}(\Omega)} I_{\mu}(u)<\frac{S}{2^{2 / n}}
$$

for $\mu$ large enough, and the existence of a solution to (1.1) follows.

Note that in striking contrast with what happens in the Dirichlet case, where the maximum of concentrated solutions cannot approach the boundary, Adimurthi, Pacella and Yadava showed in [4] that for $\mu$ large enough, a solution $u_{\mu}$ of (1.1), which satisfies $I_{\mu}\left(u_{\mu}\right)<\frac{S}{2^{2 / n}}$, attains its maximum at only one point $y_{\mu} \in \partial \Omega$. Up to a subsequence, $\left(y_{\mu}\right)$ converges to some $y^{0} \in \partial \Omega$ as $\mu$ goes to infinity, and $u_{\mu}$ satisfies

$$
\left|\nabla u_{\mu}\right|_{\mu \rightarrow+\infty}^{2} \frac{S^{n / 2}}{2} \delta_{y^{0}}
$$

in the sense of measures in $\Omega$.

Furthermore, they proved that if $n \geq 7$ and $u_{\mu}$ minimizes $I_{\mu},\left(y_{\mu}\right)$ is a maximizing sequence for the mean curvature $\bar{H}$ of the boundary $\partial \Omega$ of $\Omega$. Dropping the assumption that $u_{\mu}$ is a minimizing sequence, it is proved in [5] that the accumulation points of $\left(y_{\mu}\right)$ are more generally critical with respect to $H$, and precise $L^{\infty}$-estimates are obtained.

Some partial converse results are also available. Firsteval, we know from [4] that for $n \geq 7$ and any $y^{0} \in \partial \Omega$ such that $H\left(y^{0}\right)>0$ is a strict local maximum of $H$ on $\partial \Omega$, there exists, for $\mu$ large enough, a solution $u_{\mu}$ of (1.1), which satisfies 
$I_{\mu}\left(u_{\mu}\right)<\frac{S}{2^{2 / n}}$ and concentrates at $y^{0}$ as $\mu$ goes to infinity. In [3], Adimurthi, Mancini and Yadava showed that the same result holds assuming that $n \geq 6$ and $y^{0} \in \partial \Omega$ is a nondegenerate critical point of $H$, with $H\left(y^{0}\right)>0$.

The aim of this paper is, adapting to the present context the methods that we previously developped to analyze similar Dirichlet problems - see, for instance [17] [18] [8] - to give a general setting for the study of problems as (1.1), whatever the boundary conditions may be. The interesting point is that, although the results are qualitatively different, the same kind of arguments allows to conclude. On one hand, we find again the above results, with some extensions and improvements ; on the other hand, we look for solutions which concentrate at several points at the same time, and we prove that, in contrast with what happened in the Dirichlet case, the interactions between the different concentrated peaks remain neglictable with respect to the boundary effects induced by the positive mean curvature. Therefore, such solutions behave as a superposition of single concentrated solutions.

Namely, we state :

\section{Theorem $1.1-$}

a) Let $n \geq 7$ and $u_{\mu}$ be a solution of (1.1) such that $I_{\mu}\left(u_{\mu}\right)<\frac{S}{2^{2 / n}}$. Let $y^{0} \in \partial \Omega$ be the point at which, up to a subsequence, $u_{\mu}$ concentrates, as $\mu$ goes to infinity (in the sense $\left|\nabla u_{\mu}\right|_{\mu \rightarrow+\infty}^{2} \frac{S^{n / 2}}{2} \delta_{y^{0}}$, or $y^{0}$ is an accumulation point of the sequence $\left(y_{\mu}\right)$ such that $u_{\mu}$ attains its maximum at $\left.y_{\mu}\right)$. Then

$$
H\left(y^{0}\right) \geq 0
$$

and

$$
\text { if } H\left(y^{0}\right)>0, H^{\prime}\left(y^{0}\right)=0 \text {. }
$$

b) Let $n \geq 6$ and $y^{0} \in \partial \Omega$ be a nondegenerate critical point of $H, H\left(y^{0}\right)>0$. For $\mu$ large enough, (1.1) admits a solution $u_{\mu}$ which concentrates at $y^{0}$ (in the previous sense) as $\mu$ goes to infinity.

In fact, the nondegeneracy assumption way be weakened in the following way. Denoting

$$
H^{a}=\{y \in \partial \Omega / H(y)<a\}
$$

the level sets of $H$, we have

Theorem 1.2 - Let $n \geq 5$, and assume that $a>0$ is a critical value of $H$ such that the relative topology $\left(H^{a+\delta}, H^{a-\delta}\right)$ is nontrivial for any $\delta>0$ sufficiently small. 
There exists a solution $u_{\mu}$ of (1.1), which concentrates, as $\mu$ goes to infinity, at a point $y^{0} \in \partial \Omega$ such that $H\left(y^{0}\right)=a\left(\right.$ for $n \geq 6$, we prove that $\left.H^{\prime}\left(y^{0}\right)=0\right)$.

Solutions which concentrate at one point may be glued together, and we obtain

Theorem 1.3 - Let $n \geq 6$, and $y^{1}, \cdots, y^{k} k$ distinct points of $\partial \Omega$, each one a nondegenerate critical point of $H$ such that $H\left(y^{i}\right)>0$. There exists, for $\mu$ large enough, a solution $u_{\mu}$ of (1.1) which concentrates at $y^{1}, \cdots, y^{k}$ as $\mu$ goes to infinity (in the sense $\left|\nabla u_{\mu}\right|_{\mu \rightarrow+\infty}^{2} \frac{S^{n / 2}}{2} \sum_{i=1}^{k} \delta_{y^{i}}$ ).

Of course, we could also prove that $u_{\mu}$ being a solution of (1.1) concentrating at $k$ distinct points $y^{i}$ of $\partial \Omega$ as $\mu$ goes to infinity, these points have to satisfy $H\left(y^{i}\right)>$ $0, H^{\prime}\left(y^{i}\right)=0$ (assuming, as in Theorem 1.1, $n \geq 7$ ).

Theorem 1.3 allows to state multiplicity results, in connection with the number of critical points of $H$ on

$$
H^{+}=\{y \in \partial \Omega / H(y)>0\}
$$

Theorem $1.4-$ Let $n \geq 6$. Assume that $H$ admits $k$ nondegenerate critical points on $\mathrm{H}^{+}$. For $\mu$ large enough, (1.1) admits at least $2^{k}-1$ solutions.

The nondegeneracy condition which occurs in Theorem 1.3 could be weakened, as previously, leading to multiplicity results in relation with the relative topology between the level sets of $H$. For instance, (1.1) should admit, for $\mu$ large enough, at least $2^{\text {cat }\left(H^{+}\right)}-1$ solutions, where cat $\left(H^{+}\right)$is the Ljusternik-Schnirelman category of $H^{+}$. Note that all our results are available assuming that $n \geq 5,6$ or 7 , the low dimension spaces being the most delicate cases. However, the same should hold for any $n \geq 3$.

The proof of the theorems is based upon a parametrization of $H^{1}(\Omega)$ in a neighbourhood of the potential concentrated solutions $u$ that we look for. This parametrization is performed in the next section.

In this neighbourhood, $u$ splits into a small part $v$ which is showed to be inessential, and a concentrated part which depends on a finite number of parameters, namely the points and the speeds of concentration. It is proved in Section 3 that $u$ being a solution to (1.1) is equivalent to the fulfilment of a finite dimensional system, whose study will give us the announced results in Section 4.

The proofs require some technical results which, for the convenience of the reader, may be found in appendix. A serious amont of computations is also delayed until 
the end of the paper, so that the main arguments appear more clearly. However, and unfortunately, these computations are as heavy as they are impossible to avoid to make the demonstration rigorous.

\section{2 - THE FRAMEWORK}

Here and throughout the sequel, we assume that $n \geq 3$, and $p=\frac{n+2}{n-2}$.

We proceed to a suitable parametrization of the variational problem that we are interested in. Let us introduce the following notations.

For $k \in \mathbb{N}^{*}, \alpha=\left(\alpha_{1}, \cdots, \alpha_{k}\right) \in \mathbb{R}_{+}^{k}, \lambda=\left(\lambda_{1}, \cdots, \lambda_{k}\right) \in\left(\mathbb{R}_{+}^{*}\right)^{k}, y=\left(y^{1}, \cdots, y^{k}\right) \in$ $(\partial \Omega)^{k}$, we set

$$
\varphi(\alpha, \lambda, y)=\varphi_{\alpha, \lambda, y}=\sum_{i=1}^{k} \alpha_{i} U_{i}
$$

where $U_{i}=U_{\lambda_{i}, y^{i}}$ and $U_{\lambda_{i}, y^{i}}$ is defined by (1.3). For $\epsilon>0, B_{\epsilon}$ denotes the subset of $\left\{(\alpha, \lambda, y) \in \mathbb{R}_{+}^{k} \times\left(\mathbb{R}_{+}^{*}\right)^{k} \times(\partial \Omega)^{k}\right\}$ such that

$$
\left\{\begin{array}{l}
\frac{\bar{\alpha}}{2}<\alpha_{i}<2 \bar{\alpha}, \text { with } \bar{\alpha}=(n(n-2))^{\frac{n-2}{4}} \\
\lambda_{i}>\frac{1}{\epsilon} \quad 1 \leq i \leq k \\
\left|y^{i}-y^{j}\right|^{2}>\epsilon \quad 1 \leq i<j \leq k
\end{array}\right.
$$

We define

$$
\begin{aligned}
V_{\epsilon}= & \left\{u \in H^{1}(\Omega) / \exists(\lambda, y) \in\left(\mathbb{R}_{+}^{*}\right)^{k} \times(\partial \Omega)^{k}\right. \text { s.t. } \\
& \left.(\bar{\alpha}, \lambda, y) \in B_{\epsilon} \text { and }|\nabla(u-\varphi(\bar{\alpha}, \lambda, y))|_{2}<\epsilon\right\}
\end{aligned}
$$

with the notation $\bar{\alpha}=(\bar{\alpha}, \cdots, \bar{\alpha})$.

We have the following proposition :

Proposition 2.1 - Let $k \in \mathbb{N}^{*}$. There exists $\epsilon_{0}>0$ such that for any $\epsilon, 0<\epsilon \leq \epsilon_{0}$, and any $u \in V_{\epsilon}$, the problem

$$
\underset{(\alpha, \lambda, y) \in B_{4 \epsilon}}{\operatorname{Minimize}}|\nabla(u-\varphi(\alpha, \lambda, y))|_{2}
$$

has a unique solution (up to permutations of the indices). 
This statement is a direct adaptation to our case of [7, Proposition 7]. Its proof is given in Appendix A.

Once the unicity of the solution of the minimization problem is ensured, it is not difficult to see that it actually induces a diffeomorphism between $V_{\epsilon_{0}}$ and an open subset $M^{\prime}$ of the manifold

$$
\begin{aligned}
M=\left\{(\alpha, \lambda, y, v) \in \mathbb{R}^{k} \times\left(\mathbb{R}_{+}^{*}\right)^{k} \times(\partial \Omega)^{k} \times H^{1}(\Omega)\right. \\
\\
\left.(\alpha, \lambda, y) \in B_{\epsilon_{0}}, v \in E_{\lambda, y},|\nabla v|_{2}<\epsilon_{0}\right\}
\end{aligned}
$$

with

$$
\begin{aligned}
E_{\lambda, y}= & \left\{v \in H^{1}(\Omega) / \forall(i, j), 1 \leq i \leq k, 1 \leq j \leq n\right. \\
& \left.\int_{\Omega} \nabla v \cdot \nabla U_{i}=\int_{\Omega} \nabla v \cdot \nabla \frac{\partial U_{i}}{\partial \lambda_{i}}=\int_{\Omega} \nabla v \cdot \nabla \frac{\partial U_{i}}{\partial y_{j}^{i}}=0\right\} .
\end{aligned}
$$

$M^{\prime}$ clearly contains some open subset $N$

$$
\begin{aligned}
N= & \left\{(\alpha, \lambda, y, v) \in \mathbb{R}^{k} \times\left(\mathbb{R}_{+}^{*}\right)^{k} \times(\partial \Omega)^{k} \times H^{1}(\Omega)\right. \text { s.t. } \\
& \left|\alpha_{i}-\bar{\alpha}\right|<\eta_{0}, \lambda_{i}>\frac{1}{\eta_{0}}, \forall i ;\left|y^{i}-y^{j}\right|>d_{0}, \forall i, j, i \neq j ; \\
& \left.v \in E_{\lambda, y},|\nabla v|_{2}<\eta_{0}\right\}
\end{aligned}
$$

for some constants $\eta_{0}>0, d_{0}>0$ (we note that, as $d, d_{0}$ may be chosen as small as we want, provided that $\eta_{0}>0$ is then taken sufficiently small).

Let us now consider the variational problem. The 0 -homogeneity of $I_{\mu}$ with respect to $u$, which induces a simplified analysis in case of a single point of concentration, is not an advantage in the case of several points of concentration that we are interested in. Rather than $I_{\mu}$, we consider the functional

$$
J_{\mu}(u)=\frac{1}{2} \int_{\Omega}|\nabla u|^{2}+\frac{\mu}{2} \int_{\Omega} u^{2}-\frac{1}{p+1} \int_{\Omega}|u|^{p+1} \quad u \in H^{1}(\Omega)
$$

whose positive critical points are solutions to (1.1). We define on $N$ the corresponding functional

$$
\begin{aligned}
K_{\mu}: N & \rightarrow \mathbb{R} \\
(\alpha, \lambda, y, v) & \mapsto K_{\mu}\left(\sum_{i=1}^{k} \alpha_{i} U_{i}+v\right) .
\end{aligned}
$$


$(\alpha, \lambda, y, v)$ is a critical point of $K_{\mu}$ on $N$ if and only if $u=\sum_{i=1}^{k} \alpha_{i} U_{i}+v$ is a critical point of $J_{\mu}$ on $H^{1}(\Omega)$. We set, for $1 \leq i \leq k$ and $1 \leq j \leq n-1$

$$
\left\{\begin{aligned}
\phi_{i}(\alpha, \lambda, y, v) & =\int_{\Omega} \nabla v \cdot \nabla U_{i} \\
\psi_{i}(\alpha, \lambda, y, v) & =\int_{\Omega} \nabla v \cdot \nabla \frac{\partial U_{i}}{\partial \lambda_{i}} \\
\xi_{i, j}(\alpha, \lambda, y, v) & =\int_{\Omega} \nabla v \cdot \nabla \frac{\partial U_{i}}{\partial \tau_{j}^{i}}
\end{aligned}\right.
$$

where the $\tau_{j}^{i \prime} s, 1 \leq j \leq n-1$, build an orthonormal system of coordinates on the tangent space to $\partial \Omega$ at $y^{i}$. Considering $K_{\mu}, \phi_{i}, \psi_{i}, \xi_{i, j}$ as functionals defined on an open subset of $\mathbb{R}_{+}^{k} \times\left(\mathbb{R}_{+}^{*}\right)^{k} \times(\partial \Omega)^{k} \times H^{1}(\Omega),(\alpha, \lambda, y, v)$ will be a critical point of $K_{\mu}$ restricted to $N$ if and only if there exists $A=\left(A_{i}\right)_{1 \leq i \leq k} \in \mathbb{R}^{k}, B=\left(B_{i}\right)_{1 \leq i \leq k} \in$ $\mathbb{R}^{k}, C=\left(C_{i, j}\right) \underset{\substack{1 \leq i \leq k \\ 1 \leq j \leq n-1}}{\in} \in \mathbb{R}^{(n-1) k}$ such that

$$
K_{\mu}^{\prime}=\sum_{i=1}^{k}\left(A_{i} \phi_{i}^{\prime}+B_{i} \psi_{i}^{\prime}+\sum_{j=1}^{n-1} C_{i, j} \xi_{i, j}^{\prime}\right)
$$

The next section is devoted to a careful analysis of this equation.

\section{3 - AnAlysis of the SYSTEM}

We are going to solve equation (2.10) step by step, beginning with the variable $v$.

In order to make the statements simpler, we assume that

$$
\frac{\lambda_{i}}{\lambda_{j}} \leq \kappa_{1} \quad 1 \leq i, j \leq k
$$

where $\kappa_{1}$ is a strictly positive constant, to be determined later. This assumption is not restrictive, since it will turn out that (3.1) always holds for the solutions that we look for. For sake of simplicity, we shall also denote, in the following, by $0(f(\lambda))$ a quantity which is dominated by $\sum_{i=1}^{k} f\left(\lambda_{i}\right)$ as the $\lambda_{i}^{\prime} s$ go to infinity.

Lastly, for $v, w$ in $H^{1}(\Omega)$, we set

$$
\left\{\begin{aligned}
<v, w> & =\int_{\Omega} \nabla v \cdot \nabla w+\mu \int_{\Omega} v w \\
\|v\|^{2} & =<v, v>
\end{aligned}\right.
$$




\subsection{The $v$-part of $u$.}

Here is the main statement of this subsection.

Proposition 3.1 - There exists $\mu_{0}>0, \delta_{0}>0$, and a smooth map which to any $\mu>\mu_{0}$ and $(\alpha, \lambda, y)$ - such that $(\alpha, \lambda, y, 0) \in N$, (3.1) holds, and, for $1 \leq i \leq k$

$$
\left\{\begin{array}{l}
\inf \left(\frac{\mu}{\lambda_{i}^{\frac{3}{2}}}, \frac{\mu}{\lambda_{i}^{\frac{7}{4}}}+\frac{\mu^{\frac{1}{2}}}{\lambda_{i}^{\frac{5}{4}}}\right)<\delta_{0} \text { if } n=5 \\
\frac{\mu\left(\log \lambda_{i}\right)^{\frac{2}{3}}}{\lambda_{i}^{2}}<\delta_{0} \text { if } n=6 \\
\frac{\mu}{\lambda_{i}^{2}}<\delta_{0} \text { if } n \geq 7
\end{array}\right.
$$

- associates $\bar{v} \in E_{\lambda, y}$ such that, at the point $(\alpha, \lambda, y, \bar{v})$

$$
\frac{\partial K_{\mu}}{\partial v}=\sum_{i=1}^{k}\left(A_{i} \frac{\partial \phi_{i}}{\partial v}+B_{i} \frac{\partial \psi_{i}}{\partial v}+\sum_{j=1}^{n-1} C_{i, j} \frac{\partial \xi_{i, j}}{\partial v}\right)
$$

where $A_{i}, B_{i}, C_{i, j}$ are some real numbers depending on $\alpha, \lambda, y, \mu$.

Moreover, there exists $\nu_{0}>0$, independent of $\alpha, \lambda, y, \mu$ such that such a $\bar{v}$ is unique in $\left\{v \in E_{\lambda, y} /|v|_{H^{1}}<\nu_{0}\right\}$, and we have the estimate

$$
\begin{aligned}
\|\bar{v}\|= & 0\left[\left(\frac{1}{\lambda}+\inf \left(\frac{\mu}{\lambda^{3 / 2}}, \frac{\mu}{\lambda^{7 / 4}}+\frac{\mu^{1 / 2}}{\lambda^{5 / 4}}\right)\right) \text { if } n=5 ;\right. \\
& \left.\left(\frac{1}{\lambda}+\mu \frac{(\log \lambda)^{2 / 3}}{\lambda^{2}}\right) \text { if } n=6 ;\left(\frac{1}{\lambda}+\frac{\mu}{\lambda^{2}}\right) \text { if } n \geq 7\right]
\end{aligned}
$$

\section{Proof of the proposition.}

We expand $K_{\mu}(\alpha, \lambda, y, v)$ with respect to $v$ in a neighbourhood of 0 . According to the definition of $K_{\mu}$, we find

$$
K_{\mu}(\alpha, \lambda, y, v)=K_{\mu}(\alpha, \lambda, y, 0)+L_{\mu, \alpha, \lambda, y}(v)+Q_{\mu, \alpha, \lambda, y}(v)+R_{\mu, \alpha, \lambda, y}(v)
$$

with

$$
L_{\mu, \alpha, \lambda, y}(v)=\mu \int_{\Omega} \varphi_{\alpha, \lambda, y} v-\int_{\Omega} \varphi_{\alpha, \lambda, y}^{p} v
$$




$$
Q_{\mu, \alpha, \lambda, y}(v)=\frac{1}{2}\left(\int_{\Omega}|\nabla v|^{2}+\mu \int_{\Omega} v^{2}-p \int_{\Omega} \varphi_{\alpha, \lambda, y}^{p-1} v^{2}\right)
$$

and

$$
R_{\mu, \alpha, \lambda, y}(v)=0\left(\|v\|^{\min (3, p+1)}\right) .
$$

Moreover, the derivatives of $R_{\mu, \alpha, \lambda, y}$ satisfy

$$
R_{\mu, \alpha, \lambda, y}^{\prime}(v)=0\left(\|v\|^{\min (2, p)}\right) ; R_{\mu, \alpha, \lambda, y}^{\prime \prime}(v)=0\left(\|v\|^{\min (1, p-1)}\right)
$$

uniformly with respect to $\mu, \alpha, \lambda, y$ verifying the assumptions of the proposition.

At this stage, we need the following crucial result, about the uniform coercivity of the quadratic form $Q_{\mu, \alpha, \lambda, y}$.

Lemma 3.1 - There exists $\rho>0$ such that for any $\mu$ and $(\alpha, \lambda, y)$ verifying the assumptions of Proposition 3.1 (with $\mu_{0}$ large enough, $\delta_{0}$ small enough)

$$
Q_{\mu, \alpha, \lambda, y}(v) \geq \rho\|v\|^{2} \quad \forall v \in E_{\lambda, y} .
$$

The proof of this lemma is given in Appendix B. (In fact, for $n=5,6$, we do not need (3.3) to be satisfied, but only $\frac{\mu}{\lambda_{i}^{2}}<\delta_{0}, 1 \leq i \leq k$, with $\delta_{0}$ small enough.)

We claim that $L_{\mu, \alpha, \lambda, y}$, which is a continuous linear form on $E_{\lambda, y}$ equipped with the scalar product (3.2), is going to zero as $\mu$ goes to infinity, uniformly with respect to $\alpha, \lambda, y$ satisfying the assumptions of the proposition. Writing

$$
\left\{\begin{array}{l}
L_{\mu, \alpha, \lambda, y}(v)=<\ell_{\mu, \alpha, \lambda, y}, v> \\
Q_{\mu, \alpha, \lambda, y}(v)=\frac{1}{2}<T_{\mu, \alpha, \lambda, y} v, v>
\end{array}\right.
$$

with $\ell_{\mu, \alpha, \lambda, y} \in E_{\lambda, y}$ and $T_{\mu, \alpha, \lambda, y} \in \mathcal{L}\left(E_{\lambda, y}\right)$ a continuous, symmetric and coercive operator whose modulus of coercivity is bounded from below, the derivative of $K_{\mu}$ with respect to $v$ on $E_{\lambda, y}$ may be written as

$$
\ell_{\mu, \alpha, \lambda, y}+T_{\mu, \alpha, \lambda, y} v+0\left(\|v\|^{\min (2, p)}\right)
$$

and the second derivative is equal to

$$
T_{\mu, \alpha, \lambda, y}+0\left(\|v\|^{\min (1, p-1)}\right) .
$$


Therefore, the implicit functions theorem provides us with a smooth map which to any $\mu$ and $(\alpha, \lambda, y)$ as in the statement of Proposition 3.1, with $\mu_{0}$ large enough, associates $\bar{v} \in E_{\lambda, y}$ such that (3.4) is satisfied. Moreover, we have the estimate

$$
\|\bar{v}\|=0\left(\left\|\ell_{\mu, \alpha, \lambda, y}\right\|\right)
$$

and $\bar{v}$ is the unique solution of (3.4) in a suitable neighbourhood of zero in $E_{\lambda, y}$.

It only remains to prove the claim which, through (3.14), will also provide us with estimate (3.5). Using Hölder's inequality and Sobolev embedding theorem, we have, for any $v \in E_{\lambda, y}$

$$
\mu \int_{\Omega} \varphi_{\alpha, \lambda, y} v=0\left[\mu\left(\int_{\Omega}|v|^{p+1}\right)^{\frac{1}{p+1}} \sum_{i}\left(\int_{\Omega} U_{i}^{\frac{p+1}{p}}\right)^{\frac{p}{p+1}}\right] .
$$

From the computations in Appendix D - see (D.36) - and (3.1), we deduce that

$$
\begin{aligned}
& \mu \int_{\Omega} \varphi_{\alpha, \lambda, y} v \\
& =0\left[\|v\|\left(\frac{\mu}{\lambda^{3 / 2}} \text { if } n=5 ; \frac{\mu(\log \lambda)^{2 / 3}}{\lambda^{2}} \text { if } n=6 ; \frac{\mu}{\lambda^{2}} \text { if } n \geq 7\right)\right] .
\end{aligned}
$$

The estimate for $n=5\left(p=\frac{7}{3}\right)$ may be improved in the following way. We write

$$
\begin{aligned}
\mu \int_{\Omega} \varphi_{\alpha, \lambda, y} v= & {\left[\mu \sum_{i} \int_{\Omega \cap B\left(y^{i}, \frac{1}{\lambda_{i}^{1 / 2}}\right)} U_{i} v+\mu \sum_{i} \int_{\Omega \backslash B\left(y^{i}, \frac{1}{\lambda_{i}^{1 / 2}}\right)} U_{i} v\right] } \\
= & 0\left[\mu\left(\int_{\Omega}|v|^{10 / 3}\right)^{\frac{3}{10}} \sum_{i}\left(\int_{\Omega \cap B\left(y^{i}, \frac{1}{\lambda_{i}^{1 / 2}}\right)} U_{i}^{\frac{10}{7}}\right)^{7 / 10}\right. \\
& \left.+\mu\left(\int_{\Omega} v^{2}\right)^{1 / 2} \sum_{i}\left(\int_{\Omega \backslash B\left(y^{i}, \frac{1}{\lambda_{i}^{1 / 2}}\right)} U_{i}^{2}\right)^{1 / 2}\right] \\
= & 0\left(\frac{\mu\|v\|}{\lambda^{7 / 4}}+\frac{\mu|v|_{2}}{\lambda^{5 / 4}}\right)
\end{aligned}
$$

according to (D.24,36). Noticing that, in view of (3.2), $\mu|v|_{2} \leq \mu^{1 / 2}\|v\|$, we obtain

$$
\mu \int_{\Omega} \varphi_{\alpha, \lambda, y} v=0\left[\left(\frac{\mu}{\lambda^{7 / 4}}+\frac{\mu^{1 / 2}}{\lambda^{5 / 4}}\right)\|v\|\right] \text { for } n=5 .
$$


In order to estimate the second term in (3.7), we expand

$$
\varphi_{\alpha, \lambda, y}^{p}=\sum_{i} \alpha_{i}^{p} U_{i}^{p}+0\left(\sum_{\substack{i, j \\ i \neq j}} U_{i}^{p-1} U_{j}\right)
$$

On one hand, denoting by $B_{\ell}$ the ball of center $y^{\ell}$ and radius $d_{0} / 2$, we have, in view of (1.3)

$$
\begin{aligned}
& \int_{\Omega} U_{i}^{p-1} U_{j} v \\
& =\int_{B_{i}} U_{i}^{p-1} U_{j} v+\int_{B_{j}} U_{i}^{p-1} U_{j} v+\int_{\Omega \backslash B_{i} \cup B_{j}} U_{i}^{p-1} U_{j} v \\
& =0\left(\frac{1}{\lambda_{j}^{\frac{n-2}{2}}} \int_{B_{i}} U_{i}^{p-1}|v|+\frac{1}{\lambda_{i}^{2}} \int_{\Omega} U_{j}|v|+\frac{1}{\lambda_{i}^{2} \lambda_{j}^{\frac{n-2}{2}}} \int_{\Omega \backslash B_{i} \cup B_{j}}|v|\right) \\
& =0\left[|v|_{H^{1}}\left(\frac{1}{\lambda_{j}^{\frac{n-2}{2}}}\left(\int_{B_{i}} U_{i}^{\frac{(p-1)(p+1)}{p}}\right)^{\frac{p}{p+1}}+\frac{1}{\lambda_{i}^{2}}\left(\int_{B_{j}} U_{j}^{\frac{(p+1)}{p}}\right)^{\frac{p}{p+1}}+\frac{1}{\lambda_{i}^{2} \lambda_{j}^{\frac{n-2}{2}}}\right)\right]
\end{aligned}
$$

using Hölder's inequalities and Sobolev embedding theorem. Estimates (D.36,38) and (3.1) yield

$$
\int_{\Omega} U_{i}^{p-1} U_{j} v=0\left[|v|_{H^{1}}\left(\frac{1}{\lambda^{3}} \text { if } n=5 ; \frac{(\log \lambda)^{4 / 3}}{\lambda^{4}} \text { if } n=6 ; \frac{1}{\lambda^{4}} \text { if } n \geq 7\right)\right]
$$

On the other hand

$$
\begin{aligned}
\int_{\Omega} U_{i}^{p} v & =-\frac{1}{n(n-2)} \int_{\Omega} \Delta U_{i} \cdot v & & \text { because of }(1.2-4) \\
& =-\frac{1}{n(n-2)} \int_{\partial \Omega} \frac{\partial U_{i}}{\partial v} \cdot v & & \text { integrating by parts, since } v \in E_{\lambda, y} .
\end{aligned}
$$

Using the embedding of $H^{1}(\Omega)$ into $L^{\frac{2(n-1)}{n-2}}(\partial \Omega)$ and Hölder's inequality, we obtain

$$
\int_{\Omega} U_{i}^{p} v=0\left[|v|_{H^{1}}\left(\int_{\partial \Omega}\left|\frac{\partial U_{i}}{\partial \nu}\right|^{\frac{2(n-1)}{n}}\right)^{\frac{n}{2(n-1)}}\right] .
$$

There for, estimate (D.49) shows that

$$
\int_{\Omega} U_{i}^{p} v=0\left(\frac{|v|_{H^{1}}}{\lambda}\right)
$$


From (3.15-19), we deduce that

$$
\begin{aligned}
\left\|\ell_{\mu, \alpha, \lambda, y}\right\|=0\left[\left(\frac{1}{\lambda}+\inf \left(\frac{\mu}{\lambda^{3 / 2}}, \frac{\mu}{\lambda^{7 / 4}}+\frac{\mu^{1 / 2}}{\lambda^{5 / 4}}\right)\right) \text { if } n=5 ;\right. \\
\left.\left(\frac{\mu}{\lambda}+\mu \frac{(\log \lambda)^{2 / 3}}{\lambda^{2}}\right) \text { if } n=6 ;\left(\frac{1}{\lambda}+\frac{\mu}{\lambda^{2}}\right) \text { if } n \geq 7\right]
\end{aligned}
$$

The claim is proved and (3.14) makes the proof of Proposition 3.1 complete.

Before ending this section, let us show how the coefficients $A_{i}, B_{i}, C_{i, j}$ which occur in (3.4) may be estimated. According to the definition of $K_{\mu}, \phi_{i}, \psi_{i}, \xi_{i, j},(3.4)$ means that for any $w \in H^{1}(\Omega)$

$$
\begin{aligned}
& \int_{\Omega} \nabla\left(\varphi_{\alpha, \lambda, y}+\bar{v}\right) \cdot \nabla w+\mu \int_{\Omega}\left(\varphi_{\alpha, \lambda, y}+\bar{v}\right) w-\int_{\Omega}\left|\varphi_{\alpha, \lambda, y}+\bar{v}\right|^{p-1}\left(\varphi_{\alpha, \lambda, y}+\bar{v}\right) w \\
& =\sum_{i=1}^{k}\left(A_{i} \int_{\Omega} \nabla U_{i} \cdot \nabla w+B_{i} \int_{\Omega} \nabla \frac{\partial U_{i}}{\partial \lambda_{i}} \cdot \nabla w+\sum_{j=1}^{n-1} C_{i, j} \int_{\Omega} \nabla \frac{\partial U_{i}}{\partial \tau_{j}^{i}} \cdot \nabla w\right)
\end{aligned}
$$

Taking successively $w=U_{i}, \frac{\partial U_{i}}{\partial \lambda_{i}}, \frac{\partial U_{i}}{\partial \tau_{j}^{2}}, 1 \leq j \leq n-1$, we obtain $k(n+1)$ equations which build a linear system for the $k(n+1)$ coeffcicients $A_{i}, B_{i}, C_{i, j}$. This system is nearly diagonal, since the functions $U_{i}, \frac{\partial U_{i}}{\partial \lambda_{i}}, \frac{\partial U_{i}}{\partial \tau_{j}^{i}}$ are nearly orthogonal for the bilinear form $(v, w) \mapsto \int_{\Omega} \nabla v . \nabla w$ - see estimates (D.26). As a consequence, the system is invertible, and computing the terms on the left hand side, which are equal to

$$
\frac{\partial K_{\mu}}{\partial v} \cdot U_{i}, \frac{\partial K_{\mu}}{\partial v} \cdot \frac{\partial U_{i}}{\partial \lambda_{i}}, \frac{\partial K_{\mu}}{\partial v} \cdot \frac{\partial U_{i}}{\partial \tau_{j}^{i}} \quad \begin{aligned}
& 1 \leq i \leq k \\
& 1 \leq j \leq n-1
\end{aligned}
$$

respectively, we obtain an estimate of $A_{i}, B_{i}, C_{i j}$. The result is given in Appendix $\mathrm{C}$, Lemma C.2.

\section{3 - OPtIMIZATION With RESPECT TO $\alpha$ AND $\lambda$}

Once the functional $K_{\mu}$ has been minimized with respect to $v$, we set

$$
\widetilde{K}_{\mu}(\alpha, \lambda, y)=K_{\mu}(\alpha, \lambda, y, \bar{v})
$$

which is defined, for $\mu>\mu_{0}$, on

$$
N_{\mu}=\left\{(\alpha, \lambda, y) \in \mathbb{R}^{k} \times\left(\mathbb{R}_{+}^{k}\right)^{k} \times(\partial \Omega)^{k} /(\alpha, \lambda, y, 0) \in N,(3.1) \text { and (3.3) hold }\right\} \text {. }
$$


In order to determine the critical points of $\widetilde{K}_{\mu}$ on $N_{\mu}$, we consider the derivatives of $\widetilde{K}_{\mu}$ with respect to $\alpha$ and $\lambda$. Our aim in this subsection is to find, for given $y \in(\partial \Omega)^{k}$ - such that $\left|y^{i}-y^{j}\right|>d_{0}, 1 \leq i, j \leq k-(\alpha, \lambda) \in \mathbb{R}_{+}^{k} \times\left(\mathbb{R}_{+}^{*}\right)^{k}$ such that $(\alpha, \lambda, y) \in N_{\mu}$, and

$$
\begin{cases}\frac{\partial \widetilde{K}_{\mu}}{\partial \alpha_{i}}=\frac{\partial K_{\mu}}{\partial \alpha_{i}}+\frac{\partial K_{\mu}}{\partial v} \cdot \frac{\partial \bar{v}}{\partial \alpha_{i}}=0 & \\ \frac{\partial \widetilde{K}_{\mu}}{\partial \lambda_{i}}=\frac{\partial K_{\mu}}{\partial \lambda_{i}}+\frac{\partial K_{\mu}}{\partial v} \cdot \frac{\partial \bar{v}}{\partial \lambda_{i}}=0 & 1 \leq i \leq k .\end{cases}
$$

Note that, in this subsection, we assume that

$$
\mu<\kappa_{2} \lambda_{i} \quad 1 \leq i \leq k
$$

where $\kappa_{2}>0$ is a constant to be determined later. This assumption, which makes the computations and the statements clearer, will appear to be unrestrictive for our purposes. Then, (3.5) turns out to be

$$
\|\bar{v}\|=0\left(\frac{1}{\lambda^{3 / 4}} \text { if } n=5 ; \frac{(\log \lambda)^{\frac{2}{3}}}{\lambda} \text { if } n=6 ; \frac{1}{\lambda} \text { if } n \geq 7\right) .
$$

From the definition of $K_{\mu}$ and (3.24), computations show - see Lemma C.1 in appendix

$$
\left\{\begin{array}{l}
\frac{\partial K_{\mu}}{\partial \alpha_{i}}(\alpha, \lambda, y, \bar{v})=-C_{6} \eta_{i}-C_{7} \frac{H\left(y^{i}\right)}{\lambda_{i}} \\
+0\left[\eta_{i}^{2}+\left(\frac{1}{\lambda^{3 / 2}} \text { if } n=5 ; \frac{(\log \lambda)^{\frac{4}{3}}}{\lambda^{2}} \text { if } n=6 ; \frac{1}{\lambda^{2}} \text { if } n \geq 7\right)\right] \\
\frac{\partial K_{\mu}}{\partial \lambda_{i}}(\alpha, \lambda, y, \bar{v})=C_{8} \frac{H\left(y^{i}\right)}{\lambda_{i}^{2}}-C_{9} \frac{\mu}{\lambda_{i}^{3}} \\
+0\left[\frac{\left|\eta_{i}\right|}{\lambda_{i}^{2}}+\left(\frac{1}{\lambda^{5 / 2}} \text { if } n=5 ; \frac{(\log \lambda)^{\frac{4}{3}}}{\lambda^{3}} \text { if } n=6 ; \frac{1}{\lambda^{3}} \text { if } n \geq 7\right)\right]
\end{array}\right.
$$

with

$$
\eta_{i}=\frac{\alpha_{i}}{\bar{\alpha}}-1
$$

and $C_{6}, C_{7}, C_{8}, C_{9}$ are strictly positive constants which depends on $n$ only. Let us now estimate the remaining quantities, $\frac{\partial K_{\mu}}{\partial v} \cdot \frac{\partial \bar{v}}{\partial \alpha_{i}}$ and $\frac{\partial K_{\mu}}{\partial v} \cdot \frac{\partial \bar{v}}{\partial \lambda_{i}}$. According to (2.9) 
and (3.4), we have

$$
\left\{\begin{aligned}
\frac{\partial K_{\mu}}{\partial v} \cdot \frac{\partial \bar{v}}{\partial \alpha_{i}}=\sum_{j=1}^{k}\left(A_{j} \int_{\Omega} \nabla U_{j} \cdot \nabla \frac{\partial \bar{v}}{\partial \alpha_{i}}\right. & +B_{j} \int_{\Omega} \nabla \frac{\partial U_{j}}{\partial \lambda_{j}} \cdot \nabla \frac{\partial \bar{v}}{\partial \alpha_{i}} \\
& \left.+\sum_{\ell=1}^{n-1} C_{j, \ell} \int_{\Omega} \nabla \frac{\partial U_{j}}{\partial \tau_{\ell}^{j}} \cdot \nabla \frac{\partial \bar{v}}{\partial \alpha_{i}}\right) \\
\frac{\partial K_{\mu}}{\partial v} \cdot \frac{\partial \bar{v}}{\partial \lambda_{i}}=\sum_{j=1}^{k}\left(A_{j} \int_{\Omega} \nabla U_{j} \cdot \nabla \frac{\partial \bar{v}}{\partial \lambda_{i}}\right. & +B_{j} \int_{\Omega} \nabla \frac{\partial U_{j}}{\partial \lambda_{j}} \cdot \nabla \frac{\partial \bar{v}}{\partial \lambda_{i}} \\
& \left.+\sum_{\ell=1}^{n-1} C_{j, \ell} \int_{\Omega} \nabla \frac{\partial U_{j}}{\partial \tau_{\ell}^{j}} \cdot \nabla \frac{\partial \bar{v}}{\partial \lambda_{i}}\right)
\end{aligned}\right.
$$

Since $\bar{v} \in E_{\lambda, y}$

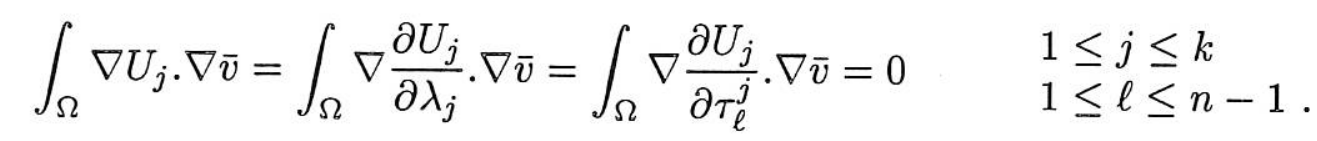

Differentiating these equalities with respect to $\alpha_{i}$, we see that they are as well satisfied with $\frac{\partial \bar{v}}{\partial \alpha_{i}}$ instead of $\bar{v}$. This means that $\frac{\partial \bar{v}}{\partial \alpha_{i}} \in E_{\lambda, y}$, and it follows from (3.26) that

$$
\frac{\partial K_{\mu}}{\partial v} \cdot \frac{\partial \bar{v}}{\partial \alpha_{i}}=0
$$

Differentiating (3.27) with respect to $\lambda_{i}$, one obtains

$$
\begin{gathered}
\int_{\Omega} \nabla U_{j} \cdot \nabla \frac{\partial \bar{v}}{\partial \lambda_{i}}=-\int_{\Omega} \nabla \frac{\partial U_{j}}{\partial \lambda_{i}} \cdot \nabla \bar{v}=0 \\
\int_{\Omega} \nabla \frac{\partial U_{j}}{\partial \lambda_{j}} \cdot \nabla \frac{\partial \bar{v}}{\partial \lambda_{i}}=-\int_{\Omega} \nabla \frac{\partial^{2} U_{j}}{\partial \lambda_{i} \partial \lambda_{j}} \cdot \nabla \bar{v}=\left\{\begin{array}{l}
0 \text { if } i \neq j \\
0\left(\frac{\|v\|}{\lambda_{i}^{2}}\right) \text { if } i=j
\end{array}\right. \\
\int_{\Omega} \bar{v} \frac{\partial U_{j}}{\partial \tau_{\ell}^{j}} \cdot \nabla \frac{\partial v}{\partial \lambda_{i}}=-\int_{\Omega} \nabla \frac{\partial^{2} U_{j}}{\partial \lambda_{i} \partial \tau_{\ell}^{j}} \cdot \nabla \bar{v}=\left\{\begin{array}{l}
0 \text { if } i \neq j \\
0(\|\bar{v}\|) \text { if } i=j
\end{array}\right.
\end{gathered}
$$

since

$$
\int_{\Omega}\left|\nabla \frac{\partial^{2} U_{i}}{\partial \lambda_{i}^{2}}\right|^{2}=0\left(\frac{1}{\lambda_{i}^{4}}\right) \text { and } \int_{\Omega}\left|\nabla \frac{\partial^{2} U_{i}}{\partial \lambda_{i} \partial y_{\ell}^{i}}\right|^{2}=0(1)
$$

as a simple computation shows - see (D.30). Therefore, (3.26), the estimate of $B_{i}, C_{i, \ell}$ given by Lemma C.2, and (3.24), imply that

$$
\frac{\partial K_{\mu}}{\partial v} \cdot \frac{\partial \bar{v}}{\partial \lambda_{i}}=0\left(\frac{1}{\lambda^{11 / 4}} \text { if } n=5 ; \frac{(\log \lambda)^{\frac{2}{3}}}{\lambda^{3}} \text { if } n=6 ; \frac{1}{\lambda^{3}} \text { if } n \geq 7\right) \text {. }
$$


Finally, from (3.26-29), we deduce

$$
\left\{\begin{aligned}
\frac{\partial \widetilde{K}_{\mu}}{\partial \alpha_{i}} & =-C_{6} \eta_{i}-C_{7} \frac{H\left(y^{i}\right)}{\lambda_{i}} \\
& +0\left[\eta_{i}^{2}+\left(\frac{1}{\lambda^{3 / 2}} \text { if } n=5 ; \frac{(\log \lambda)^{\frac{4}{3}}}{\lambda^{2}} \text { if } n=6 ; \frac{1}{\lambda^{2}} \text { if } n \geq 7\right)\right] \\
\frac{\partial \widetilde{K}_{\mu}}{\partial \lambda_{i}} & =C_{8} \frac{H\left(y^{i}\right)}{\lambda_{i}^{2}}-C_{9} \frac{\mu}{\lambda_{i}^{3}} \\
& +0\left[\frac{\left|\eta_{i}\right|}{\lambda_{i}^{2}}+\left(\frac{1}{\lambda^{5 / 2}} \text { if } n=5 ; \frac{(\log \lambda)^{\frac{4}{3}}}{\lambda^{3}} \text { if } n=6 ; \frac{1}{\lambda^{3}} \text { if } n \geq 7\right)\right] .
\end{aligned}\right.
$$

Let us assume now that

$$
H\left(y^{i}\right)>c>0 \quad 1 \leq i \leq k .
$$

With the change of variables

$$
\zeta_{i}=\frac{C_{9}}{C_{8}} \frac{\mu}{H\left(y^{i}\right)} \frac{1}{\lambda_{i}}-1 ; \xi_{i}=-C_{6} \mu \eta_{i}-C_{7} \mu H\left(y^{i}\right) \frac{1}{\lambda_{i}}
$$

(3.30) writes

$$
\left\{\begin{aligned}
\frac{\partial \widetilde{K}_{\mu}}{\partial \alpha_{i}} & =\frac{\xi_{i}}{\mu} \\
& +0\left(\frac{1}{\mu^{3 / 2}} \text { if } n=5 ; \frac{(\log \mu)^{4 / 3}}{\mu^{2}} \text { if } n=6 ; \frac{1}{\mu^{2}} \text { if } n \geq 7\right) \\
\frac{\partial \widetilde{K}_{\mu}}{\partial \lambda_{i}} & =-\frac{C_{8}^{3}}{C_{9}^{2}} \frac{H^{3}\left(y^{i}\right)}{\mu^{2}} \zeta_{i}\left(1+\zeta_{i}\right)^{2} \\
& +0\left(\frac{1}{\mu^{5 / 2}} \text { if } n=5 ; \frac{(\log \mu)^{\frac{4}{3}}}{\mu^{3}} \text { if } n=6 ; \frac{1}{\mu^{3}} \text { if } n \geq 7\right) .
\end{aligned}\right.
$$

Then, $\frac{\partial \widetilde{K}_{\mu}}{\partial \alpha_{i}}=\frac{\partial \widetilde{K}_{\mu}}{\partial \lambda_{i}}=0,1 \leq i \leq k$, turns out to be equivalent to

$$
\left\{\begin{array}{l}
\xi_{i}=V_{i}(\mu, y, \xi, \zeta) \\
\zeta_{i}=W_{i}(\mu, y, \xi, \zeta)
\end{array} \quad 1 \leq i \leq k\right.
$$

where $V_{i}, W_{i}$ are smooth functions which satisfy

$$
V_{i}, W_{i}=0\left(\frac{1}{\mu^{1 / 2}} \text { if } n=5 ; \frac{(\log \mu)^{\frac{4}{3}}}{\mu} \text { if } n=6 ; \frac{1}{\mu} \text { if } n \geq 7\right)
$$


in a neighbourhood of $\xi=\zeta=0$. Therefore, Brouwer's fixed point theorem ensures the existence, for $\mu$ large enough, of $\xi^{\mu, y}, \zeta^{\mu, y}$, such that (3.34) is satisfied. This means that for any $y \in(\partial \Omega)^{k}$ such that $\left|y^{i}-y^{j}\right|>d_{0}$ for $i \neq j$ and (3.31) holds, and any $\mu$ large enough ( $\mu$ bounded from below in function of $\mathrm{c}$ ), there exist $\alpha^{\mu, y}$ and $\lambda^{\mu, y}$ such that

$$
\left\{\begin{array}{l}
\frac{\partial \widetilde{K}_{\mu}}{\partial \alpha_{i}}\left(\alpha^{\mu, y}, \lambda^{\mu, y}, y, \bar{v}\left(\alpha^{\mu, y}, \lambda^{\mu, y}, y\right)\right)=0 \\
\frac{\partial \widetilde{K}_{\mu}}{\partial \lambda_{i}}\left(\alpha^{\mu, y}, \lambda^{\mu, y}, y, \bar{v}\left(\alpha^{\mu, y}, \lambda^{\mu, y}, y\right)\right)=0
\end{array} \quad 1 \leq i \leq k .\right.
$$

Moreover, (3.30) implies

$$
\left\{\begin{aligned}
\alpha_{i}^{\mu, y} & =\bar{\alpha}-\bar{\alpha} \frac{C_{7} C_{8}}{C_{6} C_{9}} \frac{H^{2}\left(y^{i}\right)}{\mu} \\
& +0\left(\frac{1}{\mu^{3 / 2}} \text { if } n=5 ; \frac{(\log \mu)^{4 / 3}}{\mu^{2}} \text { if } n=6 ; \frac{1}{\mu^{2}} \text { if } n \geq 7\right) \\
\lambda_{i}^{\mu, y} & =\frac{C_{9}}{C_{8}} \frac{\mu}{H\left(y^{i}\right)} \\
& +0\left(\mu^{1 / 2} \text { if } n=5 ;(\log \mu)^{4 / 3} \text { if } n=6 ; 1 \text { if } n \geq 7\right) .
\end{aligned}\right.
$$

We verify that the assumptions of Proposition 3.1 are satisfied by $\left(\mu, \alpha^{\mu, y}, \lambda^{\mu, y}, y\right)$, and

$$
\frac{\lambda_{i}^{\mu, y}}{\lambda_{j}^{\mu, y}} \leq \kappa_{1}, \mu<\kappa_{2} \lambda_{i}^{\mu, y} \quad 1 \leq i \leq k, 1 \leq j \leq k, i \neq j
$$

provided that (3.31) holds and $\mu$ is large enough, with

$$
\kappa_{1}=\frac{2 \max _{y \in \partial \Omega} H(y)}{c} ; \kappa_{2}=\frac{2 C_{8} \max _{y \in \partial \Omega} H(y)}{C_{9}} .
$$

We note that we have also, for $\mu$ large enough

$$
\kappa_{3} \lambda_{i}^{\mu, y}<\mu \quad 1 \leq i \leq k, \quad \text { with } \quad \kappa_{3}=\frac{C_{8} c}{2 C_{9}}
$$

Further computations, giving estimates of the second derivatives of $\widetilde{K}_{\mu}$ with respect to the $\alpha_{i}^{\prime} s$ and $\lambda_{i}^{\prime} s$ - see, for instance, [19, Appendix C] - show that $\left(\alpha^{\mu, y}, \lambda^{\mu, y}\right)$ such the assumptions of Proposition 3.1 and (3.35) hold is actually unique, and is a nondegenerate critical point of the map

$$
(\alpha, \lambda) \mapsto \widetilde{K}_{\mu}(\alpha, \lambda, y, \bar{v}(\alpha, \lambda, y))
$$


As a consequence, implicit function theorem provides us with a smooth map which to any $y \in(\partial \Omega)^{k}$, such that $\left|y^{i}-y^{j}\right|>d_{0}$ for $i \neq j$ and $H\left(y^{i}\right)>c$ for any $i$, associates $\left(\alpha^{\mu, y}, \lambda^{\mu, y}\right)$ such that (3.35) is satisfied, together with the estimates (3.36). (We recall that $d_{0}>0$ and $c>0$ may be chosen arbitrarily small, provided that $\mu$ is then assumed to be large enough.)

\subsection{A function which depends on $y$ only.}

$\mathrm{On}$

$$
P_{c, d_{0}}=\left\{y \in(\partial \Omega)^{k} /\left|y^{i}-y^{j}\right|>d_{0}, 1 \leq i \leq j \leq k ; H\left(y^{i}\right)>c, 1 \leq i \leq k\right\}
$$

we set

$$
\widetilde{\widetilde{K}}_{\mu}(y)=\widetilde{K}_{\mu}\left(\alpha^{\mu, y}, \lambda^{\mu, y}, y\right)=\widetilde{K}_{\mu}\left(\alpha^{\mu, y}, \lambda^{\mu, y}, y, \bar{v}\left(\alpha^{\mu, y}, \ddot{\lambda}^{\mu, y}, y\right)\right) .
$$

According to $(3.36,24)$, we have

$$
\left\|\bar{v}\left(\alpha^{\mu, y}, \lambda^{\mu, y}, y\right)\right\|=0\left(\frac{1}{\mu^{3 / 4}} \text { if } n=5 ; \frac{(\log \mu)^{\frac{2}{3}}}{\mu} \text { if } n=6 ; \frac{1}{\mu} \text { if } n \geq 7\right)
$$

and from subsection 3.1 we derive

$$
\begin{aligned}
\widetilde{K}_{\mu}\left(\alpha^{\mu, y}, \lambda^{\mu, y}, y\right) & =K_{\mu}\left(\alpha^{\mu, y}, \lambda^{\mu, y}, y, 0\right) \\
& +0\left(\frac{1}{\mu^{3 / 2}} \text { if } n=5 ; \frac{(\log \mu)^{\frac{4}{3}}}{\mu^{2}} \text { if } n=6 ; \frac{1}{\mu^{2}} \text { if } n \geq 7\right) .
\end{aligned}
$$

The definition of $K_{\mu}$ yields

$$
K_{\mu}(\alpha, \lambda, y, 0)=\frac{1}{2}\left(\int_{\Omega}\left(\left|\nabla \varphi_{\alpha, \lambda, y}\right|^{2}+\mu \varphi_{\alpha, \lambda, y}^{2}\right)\right)-\frac{1}{p+1} \int_{\Omega} \varphi_{\alpha, \lambda, y}^{p+1} .
$$

Therefore

$$
\begin{aligned}
K_{\mu}(\alpha, \lambda, y, 0) & =\frac{1}{2}\left[\sum_{i=1}^{k} \alpha_{i}^{2}\left(\int_{\Omega}\left|\nabla U_{i}\right|^{2}+\mu \int_{\Omega} U_{i}^{2}\right)\right. \\
& \left.+\sum_{\substack{1 \leq i, j \leq k \\
i \neq j}} \alpha_{i} \alpha_{j}\left(\int_{\Omega} \nabla U_{i} . \nabla U_{j}+\mu \int_{\Omega} U_{i} U_{j}\right)\right] \\
& -\frac{1}{p+1}\left[\sum_{i=1}^{k} \alpha_{i}^{p+1} \int_{\Omega} U_{i}^{p+1}+0\left(\sum_{\substack{1 \leq i, j \leq k \\
i \neq j}} \int_{\Omega} U_{i}^{p} U_{j}\right)\right]
\end{aligned}
$$


and from (D.6,17,21,25,31,42) and $(3.36,41,43)$, we deduce

$$
\begin{aligned}
\widetilde{K}_{\mu}(y)= & -\frac{(n-1)(n-3)}{4} k S^{n / 2}+\frac{C_{10}}{\mu} \sum_{i=1}^{k} H^{2}\left(y^{i}\right) \\
& +0\left(\frac{1}{\mu^{3 / 2}} \text { if } n=5 ; \frac{(\log \mu)^{\frac{4}{3}}}{\mu^{2}} \text { if } n=6 ; \frac{1}{\mu^{2}} \text { if } n \geq 7\right)
\end{aligned}
$$

with

$$
C_{10}=\frac{C_{8}}{2 C_{9}}\left((n(n-2)-1) \frac{C_{7}}{C_{6}} S^{n / 2}+\bar{\alpha}^{2}\left((n-2)^{2} C_{4}-C_{1}+2 \frac{C_{5} C_{8}}{C_{9}}\right)\right)>0 .
$$

According to the definition of $\widetilde{\widetilde{K}}_{\mu}$, we have also

$$
\frac{\partial \widetilde{\widetilde{K}}_{\mu}}{\partial \tau_{j}^{i}}=\frac{\partial K_{\mu}}{\partial \tau_{j}^{i}}+\frac{\partial K_{\mu}}{\partial v} \cdot \frac{\partial \bar{v}}{\partial \tau_{j}^{i}} \quad 1 \leq i \leq k, 1 \leq j \leq n-1
$$

at the point $\left(\alpha^{\mu, y}, \lambda^{\mu, y}, y, \bar{v}\left(\alpha^{\mu, y}, \lambda^{\mu, y}, y\right)\right)$. Similarly to (3.26), we have

$$
\begin{aligned}
\frac{\partial K_{\mu}}{\partial v} \cdot \frac{\partial \bar{v}}{\partial \tau_{j}^{i}} & =\sum_{\ell=1}^{k}\left(A_{\ell} \int_{\Omega} \nabla U_{j} \cdot \nabla \frac{\partial \bar{v}}{\partial \tau_{j}^{i}}\right. \\
& \left.+B_{\ell} \int_{\Omega} \nabla \frac{\partial U_{\ell}}{\partial \lambda_{\ell}} \cdot \nabla \frac{\partial \bar{v}}{\partial \tau_{j}^{i}}+\sum_{m=1}^{n-1} C_{\ell, m} \int_{\Omega} \nabla \frac{\partial U_{\ell}}{\partial \tau_{m}^{\ell}} \cdot \nabla \frac{\partial \bar{v}}{\partial \tau_{j}^{i}}\right)
\end{aligned}
$$

and, differentiating (3.27) with respect to $y_{j}^{i}$, we obtain

$$
\left\{\begin{array}{l}
\int_{\Omega} \nabla U_{\ell} \cdot \nabla \frac{\partial \bar{v}}{\partial y_{j}^{i}}=-\int_{\Omega} \nabla \frac{\partial U_{\ell}}{\partial y_{j}^{i}} \cdot \nabla \bar{v}=0 \\
\int_{\Omega} \nabla \frac{\partial U_{\ell}}{\partial \lambda_{\ell}} \cdot \nabla \frac{\partial \bar{v}}{\partial y_{j}^{i}}=-\int_{\Omega} \nabla \frac{\partial^{2} U_{\ell}}{\partial y_{j}^{i} \partial \lambda_{\ell}} \cdot \nabla \bar{v}=\left\{\begin{array}{l}
0 \text { if } i \neq \ell \\
0(\|\bar{v}\|) \text { if } i=\ell
\end{array}\right. \\
\int_{\Omega} \nabla \frac{\partial U_{\ell}}{\partial y_{m}^{\ell}} \cdot \nabla \frac{\partial \bar{v}}{\partial y_{j}^{i}}=-\int_{\Omega} \nabla \frac{\partial^{2} U_{\ell}}{\partial y_{j}^{i} \partial y_{m}^{\ell}} \cdot \nabla \bar{v}=\left\{\begin{array}{l}
0 \text { if } i \neq \ell \\
0\left(\lambda_{i}^{2}\|\bar{v}\|\right) \text { if } i=\ell
\end{array}\right.
\end{array}\right.
$$

since $\int_{\Omega}\left|\nabla \frac{\partial^{2} U_{i}}{\partial y_{j}^{i} \partial \lambda_{i}}\right|^{2}=0(1)$ and $\int_{\Omega}\left|\nabla \frac{\partial^{2} U_{i}}{\partial y_{j}^{i} \partial y_{m}^{i}}\right|^{2}=0\left(\lambda_{i}^{2}\right)$ - see (D.30). On the other hand, taking successively $w=U_{i}, \frac{\partial U_{i}}{\lambda y_{i}}, \frac{\partial U_{i}}{\partial \tau_{j}^{i}}, 1 \leq i \leq k, 1 \leq j \leq n-1$, in (3.20), we obtain a linear system

$$
\frac{\partial K_{\mu}}{\partial \alpha_{i}}=\sum_{\ell=1}^{k}\left(A_{\ell} \int_{\Omega} \nabla U_{\ell} \cdot \nabla U_{i}+B_{\ell} \int_{\Omega} \nabla \frac{\partial U_{\ell}}{\partial \lambda_{\ell}} \cdot \nabla U_{i}+\sum_{m=1}^{n-1} C_{\ell, m} \int_{\Omega} \nabla \frac{\partial U_{\ell}}{\partial \tau_{m}^{\ell}} . \nabla U_{i}\right)
$$




$$
\begin{aligned}
\frac{1}{\alpha_{i}} \frac{\partial K_{\mu}}{\partial \lambda_{i}} & =\sum_{\ell=1}^{k}\left(A_{\ell} \int_{\Omega} \nabla U_{\ell} \cdot \nabla \frac{\partial U_{i}}{\partial \lambda_{i}}+B_{\ell} \int_{\Omega} \nabla \frac{\partial U_{\ell}}{\partial \lambda_{\ell}} \cdot \nabla \frac{\partial U_{i}}{\partial \lambda_{i}}+\sum_{m=1}^{n-1} C_{\ell, m} \int_{\Omega} \nabla \frac{\partial U_{\ell}}{\partial \tau_{m}^{\ell}} \cdot \nabla \frac{\partial U_{i}}{\partial \lambda_{i}}\right) \\
\frac{1}{\alpha_{i}} \frac{\partial K_{\mu}}{\partial \tau_{j}^{i}} & =\sum_{\ell=1}^{k}\left(A_{\ell} \int_{\Omega} \nabla U_{\ell} \cdot \nabla \frac{\partial U_{i}}{\partial \tau_{j}^{i}}+B_{\ell} \int_{\Omega} \nabla \frac{\partial U_{\ell}}{\partial \lambda_{\ell}} \cdot \nabla \frac{\partial U_{i}}{\partial \tau_{j}^{i}}+\sum_{m=1}^{n-1} C_{\ell, m} \int_{\Omega} \nabla \frac{\partial U_{\ell}}{\partial \tau_{m}^{\ell}} \cdot \nabla \frac{\partial U_{i}}{\partial \tau_{j}^{i}}\right) .
\end{aligned}
$$

At the considered point, we have - see (3.22,28-29)

$$
\frac{\partial K_{\mu}}{\partial \alpha_{i}}=0 ; \frac{\partial K_{\mu}}{\partial \lambda_{i}}=0\left(\frac{1}{\lambda^{1 / 4}} \text { if } n=5 ; \frac{(\log \lambda)^{\frac{2}{3}}}{\lambda^{3}} \text { if } n=6 ; \frac{1}{\lambda^{3}} \text { if } n \geq 7\right)
$$

with $\lambda=\lambda^{\mu, y}$ and, according to Lemma C.1 in appendix - see (C.5,7)

$$
\frac{\partial K_{\mu}}{\partial \tau_{j}^{i}}=0\left(\frac{1}{\lambda^{1 / 2}} \text { if } n=5 ; \frac{1}{\lambda} \text { if } n \geq 6\right) .
$$

Therefore, using (D.27), we deduce from this invertible linear system that

$$
\left\{\begin{array}{l}
A_{\ell}=0\left(\frac{1}{\lambda^{3 / 2}} \text { if } n=5 ; \frac{(\log \lambda)^{\frac{2}{3}}}{\lambda^{2}} \text { if } n=6 ; \frac{1}{\lambda^{2}} \text { if } n \geq 7\right) \\
B_{\ell}=0\left(\frac{1}{\lambda^{1 / 2}} \text { if } n=5 ; \frac{(\log \lambda)^{\frac{2}{3}}}{\lambda} \text { if } n=6 ; \frac{1}{\lambda} \text { if } n \geq 7\right) \\
C_{\ell, m}=0\left(\frac{1}{\lambda^{5 / 2}} \text { if } n=5 ; \frac{(\log \lambda)^{\frac{2}{3}}}{\lambda^{3}} \text { if } n=6 ; \frac{1}{\lambda^{3}} \text { if } n \geq 7\right) .
\end{array}\right.
$$

Finally, $(3.47-49,24)$ yield

$$
\frac{\partial K_{\mu}}{\partial v} \cdot \frac{\partial \bar{v}}{\partial \tau_{j}^{i}}=0\left(\frac{1}{\lambda^{5 / 4}} \text { if } n=5 ; \frac{(\log \lambda)^{\frac{4}{3}}}{\lambda^{2}} \text { if } n=6 ; \frac{1}{\lambda^{2}} \text { if } n \geq 7\right) .
$$

This, together with (3.46) and (C.7) - see Lemma C.1 in appendix - gives, for $n \geq 6$

$$
\frac{\partial \widetilde{\widetilde{K}}_{\mu}(y)}{\partial \tau_{j}^{i}}=-\frac{C_{8}}{\lambda_{i}} \frac{\partial H}{\partial \tau_{j}^{i}}\left(y^{i}\right)+0\left(\frac{(\log \lambda)^{\frac{4}{3}}}{\lambda^{7 / 6}} \text { if } n=6 ; \frac{1}{\lambda^{\inf \left(\frac{7}{6}, \frac{n}{n-2}\right)}} \text { if } n \geq 7\right)
$$

with $\lambda=\lambda^{\mu, y}$, so that, using (3.36)

$$
\begin{aligned}
\frac{\partial \widetilde{\widetilde{K}}_{\mu}}{\partial \tau_{j}^{i}}(y) & =-\frac{C_{8}^{2}}{C_{9}} \frac{H\left(y^{i}\right)}{\mu} \frac{\partial H}{\partial \tau_{j}^{i}}\left(y^{i}\right) \\
& +0\left(\frac{(\log \mu)^{\frac{4}{3}}}{\mu^{7 / 6}} \text { if } n=6 ; \frac{1}{\mu^{\inf \left(\frac{7}{6}, \frac{n}{n-2}\right)}} \text { if } n \geq 7\right) .
\end{aligned}
$$


We are now able to prove the theorems.

\section{4 - Proof of the theorems}

\subsection{Proof of Theorem 1.1 a)}

Let $n \geq 7$ and $u_{\mu}$ be a solution of (1.1) such that

$$
I_{\mu}\left(u_{\mu}\right)=\frac{\int_{\Omega}\left|\nabla u_{\mu}\right|^{2}+\mu \int_{\Omega} u_{\mu}^{2}}{\left(\int_{\Omega}\left|u_{\mu}\right|^{p+1}\right)^{\frac{2}{p+1}}}<\frac{S}{2^{2 / n}}
$$

Multiplying (1.1) by $u_{\mu}$ and integrating on $\Omega$, we get

$$
\int_{\Omega}\left|\nabla u_{\mu}\right|^{2}+\mu \int_{\Omega} u_{\mu}^{2}=\int_{\Omega} u_{\mu}^{p+1}
$$

As a consequence, there exists $x \in \Omega$ such that $u_{\mu}(x) \geq \mu^{\frac{1}{p-1}}$, and $\max _{\bar{\Omega}} u_{\mu}$ goes to infinity as $\mu$ goes to infinity. It follows from a classical blow-up analysis - see [11], or $\left[4\right.$, Lemma 2.2] - that $\max _{\bar{\Omega}} u_{\mu}$ is attained at a unique point $y_{\mu} \in \partial \Omega$ in $\bar{\Omega}$, and

$$
\left|\nabla\left(u_{\mu}-\bar{\alpha} U_{\lambda_{\mu}, y_{\mu}}\right)\right|_{2} \rightarrow 0 \quad \text { as } \quad \mu \rightarrow+\infty
$$

with

$$
\lambda_{\mu}=\left(\frac{u_{\mu}\left(y_{\mu}\right)}{\bar{\alpha}}\right)^{\frac{2}{n-2}} \geq \frac{\mu^{1 / 2}}{\bar{\alpha}^{\frac{2}{n-2}}}
$$

Therefore, Proposition 2.1 ensures that, for $\mu$ large enough, there is a unique way to write

$$
u_{\mu}=\alpha_{\mu} U_{\lambda_{\mu}^{\prime}, y_{\mu}^{\prime}}+v_{\mu} \quad\left(\alpha_{\mu}, \lambda_{\mu}^{\prime}, y_{\mu}^{\prime}, v_{\mu}\right) \in N
$$

where $N$ is defined in (2.6). Since $\left(\alpha_{\mu}, \lambda_{\mu}^{\prime}, y_{\mu}^{\prime}\right)$ minimizes $\left|\nabla\left(u_{\mu}-\alpha U_{\lambda, y}\right)\right|_{2}$ with respect to $(\alpha, \lambda, y)$ in a set containing $\left(\bar{\alpha}, \lambda_{\mu}, y_{\mu}\right),(4.3)$ implies that

$$
\left|\nabla v_{\mu}\right|_{2} \rightarrow 0 \text { as } \quad \mu \rightarrow+\infty
$$

Then, it follows from Lemma A.1 in appendix that

$$
\alpha_{\mu} \rightarrow \bar{\alpha}, \frac{\lambda_{\mu}}{\lambda_{\mu}^{\prime}} \rightarrow 1, \lambda_{\mu} \lambda_{\mu}^{\prime}\left|y_{\mu}-y_{\mu}^{\prime}\right|^{2} \rightarrow 0 \quad \text { as } \quad \mu \rightarrow+\infty
$$


From $(4.1,2)$ we deduce

$$
\int_{\Omega}\left|\nabla u_{\mu}\right|^{2}+\mu \int_{\Omega} u_{\mu}^{2}<\frac{S^{n / 2}}{2}
$$

In view of (4.5), estimate (D.6) yields

$$
\int_{\Omega}\left|\nabla u_{\mu}\right|^{2}=\alpha_{\mu}^{2} \frac{S^{n / 2}}{2(n(n-2))^{\frac{n-2}{2}}}-\alpha_{\mu}^{2} C_{1} \frac{H\left(y_{\mu}^{\prime}\right)}{\lambda_{\mu}^{\prime}}+0\left(\frac{1}{\lambda_{\mu}^{\prime 2}}+\left|\nabla v_{\mu}\right|_{2}^{2}\right)
$$

so that, using (4.4,6-7)

$$
\int_{\Omega}\left|\nabla u_{\mu}\right|^{2}=\frac{S^{n / 2}}{2}+o(1) \quad \text { as } \quad \mu \rightarrow+\infty .
$$

As a consequence

$$
\mu \int_{\Omega} u_{\mu}^{2}=o(1) \quad \text { as } \quad \mu \rightarrow+\infty
$$

and, since, according to (D.21) and $(4.4,7)$

$$
\mu \int_{\Omega} U_{\lambda_{\mu}^{\prime}, y_{\mu}^{\prime}}^{2}=\mu \frac{C_{5}}{\lambda_{\mu}^{\prime 2}}+o\left(\frac{\mu}{\lambda_{\mu}^{\prime 3}}\right)=0(1) \quad \text { as } \quad \mu \rightarrow+\infty
$$

$(4.6,10)$ imply that

$$
\mu \int_{\Omega} v_{\mu}^{2}=0(1) \quad\left|v_{\mu}\right|_{H^{1}}=o(1) \quad \text { as } \quad \mu \rightarrow+\infty
$$

Proceeding as in Subsection 3.1, and using (4.11), we obtain

$$
\mu \int_{\Omega} u_{\mu}^{2}=\mu \alpha_{\mu}^{2} \frac{C_{5}}{\lambda_{\mu}^{\prime 2}}+\mu \int_{\Omega} v_{\mu}^{2}+0\left(\frac{\mu}{\lambda_{\mu}^{\prime 2}}\left|v_{\mu}\right|_{H^{1}}+\frac{\mu}{\lambda_{\mu}^{\prime 3}}\right) .
$$

Following again the arguments of Subsection 3.1, and using (D.17), we have also

$$
\int_{\Omega} u_{\mu}^{p+1}=\alpha_{\mu}^{p+1} \frac{S^{n / 2}}{2(n(n-2))^{\frac{n}{2}}}-\alpha_{\mu}^{p+1} C_{4} \frac{H\left(y_{\mu}^{\prime}\right)}{\lambda_{\mu}^{\prime}}+0\left(\left|v_{\mu}\right|_{H^{1}}^{2}+\frac{1}{\lambda_{\mu}^{\prime 2}}\right) .
$$

From $(4.9,12-13)$, we deduce

$$
\begin{aligned}
I_{\mu}\left(u_{\mu}\right)= & \frac{S}{2^{2 / n}}+\frac{2^{\frac{n-2}{n}}(n(n-2))^{\frac{n-2}{2}}}{S^{\frac{n-2}{2}}}\left(-C_{11} \frac{H\left(y_{\mu}^{\prime}\right)}{\lambda_{\mu}^{\prime}}+C_{5} \frac{\mu}{\lambda_{\mu}^{\prime 2}}+\frac{\mu}{\alpha_{\mu}^{2}} \int_{\Omega} v_{\mu}^{2}\right) \\
& +0\left(\frac{1}{\lambda_{\mu}^{\prime 2}}+\frac{\mu}{\lambda_{\mu}^{\prime 3}}+\frac{\mu}{\lambda_{\mu^{\prime 2}}}\left|v_{\mu}\right|_{H^{1}}^{2}+\left|v_{\mu}\right|_{H^{1}}^{2}\right)
\end{aligned}
$$


with

$$
\begin{aligned}
C_{11} & =C_{1}-(n-2)^{2} C_{4}=\frac{(n-2)^{2} \sigma_{n-2}}{2} \frac{\Gamma\left(\frac{n+1}{2}\right) \Gamma\left(\frac{n-3}{2}\right)}{\Gamma(n)}>0 \\
C_{5} & =\frac{\sigma_{n-1}}{2} \frac{\Gamma\left(\frac{n}{2}\right) \Gamma\left(\frac{n-4}{2}\right)}{\Gamma(n-2)}>0 .
\end{aligned}
$$

Therefore, as

$$
\frac{1}{\lambda_{\mu}^{\prime 2}}+\frac{\mu}{\lambda_{\mu}^{\prime 3}}+\frac{\mu}{\lambda_{\mu}^{\prime 2}}\left|v_{\mu}\right|_{H^{1}}^{2}+\left|v_{\mu}\right|_{H^{1}}^{2}=o\left(1+\frac{\mu}{\lambda_{\mu}^{\prime 2}}\right)
$$

(4.1) shows that necessarily

$$
\frac{\mu}{\lambda_{\mu}^{\prime 2}}=o(1)
$$

as $\mu$ goes to infinity. It follows from (4.15) and Proposition 3.1 that, actually

$$
v_{\mu}=\bar{v}\left(\alpha_{\mu}, \lambda_{\mu}^{\prime}, y_{\mu}^{\prime}\right)
$$

for $\mu$ large enough. (3.5) yields

$$
\left\|v_{\mu}\right\|^{2}=\int_{\Omega}\left|\nabla v_{\mu}\right|^{2}+\mu \int_{\Omega} v_{\mu}^{2}=0\left(\frac{1}{\lambda_{\mu}^{\prime 2}}+\frac{\mu^{2}}{\lambda_{\mu}^{\prime 4}}\right) .
$$

Hence, coming back to (4.14), we find

$$
I_{\mu}\left(u_{\mu}\right)=\frac{S}{2^{2 / n}}+\frac{2^{\frac{n-2}{2}}(n(n-2))^{\frac{n-2}{2}}}{S^{\frac{n-2}{2}}}\left(-C_{11} \frac{H\left(y_{\mu}^{\prime}\right)}{\lambda_{\mu}^{\prime}}+C_{5} \frac{\mu}{\lambda_{\mu}^{\prime 2}}\right)+0\left(\frac{1}{\lambda_{\mu}^{\prime 2}}+\frac{\mu^{2}}{\lambda_{\mu}^{\prime 4}}\right)
$$

Then, $(4.1,15)$ show that, necessarily

$$
H\left(y_{\mu}^{\prime}\right)>0
$$

whence, if $y^{o}$ is an accumulation point of $\left(y_{\mu}\right)$

$$
H\left(y^{o}\right) \geq 0
$$

since (4.7) shows that $y^{o}$ is also an accumulation point of $\left(y_{\mu}^{\prime}\right)$. If $H\left(y^{o}\right)>0$, we can assume that for $\mu$ large enough, $H\left(y_{\mu}^{\prime}\right)>c>0$. (4.1,15-16) ensure that, for $\mu$ large enough, we have

$$
\mu<\frac{2 C_{11} \max _{y \in \partial \Omega} H(y)}{C_{5}} \lambda_{\mu}^{\prime}=\kappa_{2} \lambda_{\mu}^{\prime} .
$$

Thus, it follows from Subsection 3.2 that

$$
\left(\alpha_{\mu}, \lambda_{\mu}^{\prime}\right)=\left(\alpha^{\mu, y_{\mu}^{\prime}}, \lambda^{\mu, y_{\mu}^{\prime}}\right)
$$

and, $u_{\mu}$ being a solution to (1.1), $y_{\mu}^{\prime}$ has to be a critical point of $\widetilde{\widetilde{K}}_{\mu}$. Then, it follows from (3.50) that $H^{\prime}\left(y^{o}\right)=0$. 


\subsection{Proof of Theorem $\mathbf{1 . 2}$}

(3.45) shows that, for $n \geq 5$ and $k=1$, up to additive and multiplicative constants, $\widetilde{\widetilde{K}}_{\mu}(y)$ is close to $H^{2}(y)$ in $L^{\infty}$-norm on

$$
H^{c}=\{y \in \partial \Omega / H(y)>c\}
$$

for any fixed $c>0$, provided that $\mu$ is large enough. Indeed, defining on $H^{c}$

$$
L_{\mu}(y)=\frac{\mu}{C_{10}}\left(\widetilde{K}_{\mu}(y)+\frac{(n-1)(n-3)}{4} S^{n / 2}\right)
$$

we have

$$
L_{\mu}(y)=H^{2}(y)+0\left(\frac{1}{\mu^{1 / 2}} \text { if } n=5 ; \frac{(\log \mu)^{4 / 3}}{\mu} \text { if } n=6 ; \frac{1}{\mu} \text { if } n \geq 7\right) .
$$

We assume that $a>0$ is a critical value for $H$ such that, for any $\delta>0$ sufficiently small, the relative topology $\left(H^{a+\delta}, H^{a-\delta}\right)$ is nontrivial. By excision, the relative topology $\left(H^{a+\delta} \cap H^{c}, H^{a-\delta} \cap H^{c}\right)$ is nontrivial, where $c>0$ is chosen such that $c<a$ (and then, $c<a-\delta$, for $\delta$ sufficiently small). (4.19) shows that for $\mu$ large enough,

$$
L_{\mu}^{(a-\delta)^{2}-c_{\mu}} \subset H^{a-\delta} \cap H^{c} \subset H^{a+\delta} \cap H^{c} \subset L_{\mu}^{(a+\delta)^{2}+c_{\mu}}
$$

with $c_{\mu}$ going to zero as $\mu$ goes to infinity. It follows that $L_{\mu}$ has a critical point between the levels $a^{2}-c_{\mu}$ and $a^{2}+c_{\mu}$. This means that, for $\mu$ large enough, (1.1) has a solution $u_{\mu}=\alpha_{\mu} U_{\lambda_{\mu}, y_{\mu}}+v_{\mu}$ which concentrates as $\mu$ goes to infinity, up to a subsequence, at a point $y^{o}=\lim _{\mu \rightarrow+\infty} y_{\mu} \in \partial \Omega$, such that $H\left(y^{o}\right)=a$. $y_{\mu}$ being a critical point of $\widetilde{\widetilde{K}}_{\mu}$, it follows from (3.50) that, if $n \geq 6, H^{\prime}\left(y^{o}\right)=0$.

\section{Remarks.}

1 - If $a$ is an isolated critical value for $H$, by intercalating the level sets of $L_{\mu}$ between the level sets of $H^{2}$, we see that in fact (1.1) admits at least as many distinct solutions $u_{\mu}$ of the previous type as the relative topology $\left(H^{a+\delta}, H^{a-\delta}\right)$ has generators.

2 - What we find is actually a critical point of $K_{\mu}$, which provides us with a solution $u_{\mu}=\alpha_{\mu} U_{\lambda_{\mu}, y_{\mu}}+v_{\mu}$ of

$$
\left\{\begin{aligned}
-\Delta u_{\mu}+\mu u_{\mu} & =\left|u_{\mu}\right|^{p-1} u_{\mu} \quad \text { in } \quad \Omega \\
\frac{\partial u_{\mu}}{\partial \nu} & =0 \text { on } \partial \Omega
\end{aligned}\right.
$$

with $y_{\mu} \in \partial \Omega$, and $\alpha_{\mu} \rightarrow \alpha, \lambda_{\mu} \rightarrow+\infty,\left\|v_{\mu}\right\| \rightarrow 0$ as $\mu \rightarrow+\infty$. Let us show that in fact $u_{\mu}>0$ in $\Omega$. First it is clear, since $\left\|v_{\mu}\right\|$ goes to zero, that $u_{\mu} \not \equiv 0$ for 
$\mu$ large enough. Secondly, multiplying the equation in $(4.20)$ by $u_{\mu}^{-}=\max \left(0,-u_{\mu}^{-}\right)$ and integrating over $\Omega$, we obtain

$$
\int_{\Omega}\left|\nabla u_{\mu}^{-}\right|^{2}+\mu \int_{\Omega}\left(u_{\mu}^{-}\right)^{2}=\int_{\Omega}\left(u_{\mu}^{-}\right)^{p+1}
$$

As, from the continuous embedding of $L^{p+1}(\Omega)$ in $H^{1}(\Omega)$, we have also

$$
\left(\int_{\Omega}\left(u_{\mu}^{-}\right)^{p+1}\right)^{\frac{2}{p+1}} \leq C_{\Omega}\left(\int_{\Omega}\left|\nabla u_{\mu}^{-}\right|^{2}+\mu \int_{\Omega}\left(u_{\mu}^{-}\right)^{2}\right)
$$

this implies

$$
\text { either } \int_{\Omega}\left(u_{\mu}^{-}\right)^{p+1} \geq \frac{1}{C_{\Omega}^{n / 2}}, \text { or } u_{\mu}^{-} \equiv 0
$$

On the other hand

$$
0 \leq u_{\mu}^{-}=\max \left(0,-\alpha_{\mu} U_{\lambda_{\mu}, y_{\mu}}-v_{\mu}\right) \leq\left|v_{\mu}\right|
$$

and $\left|v_{\mu}\right|_{p+1} \rightarrow 0$ as $\mu \rightarrow+\infty$. We conclude that for $\mu$ large enough, $u_{\mu}^{-} \equiv 0$, and $u_{\mu} \geq 0$. Since $u_{\mu}$ satisfies

$$
-\Delta u_{\mu}+\mu u_{\mu} \geq 0 \quad \text { in } \Omega ; u_{\mu} \geq 0 \quad \text { in } \Omega ; u_{\mu} \not \equiv 0
$$

the strong maximum principle ensures that $u_{\mu}>0$ in $\Omega$, and then $u_{\mu}$ is a solution to (1.1)。

\subsection{Proof of Theorems $1.1 \mathrm{~b}), 1.3,1.4$}

Since Theorems $1.1 \mathrm{~b}$ ) and 1.4 are straight forward consequences of Theorem 1.3, it is sufficient to prove this result.

Let $n \geq 6$, and $\bar{y}^{1}, \cdots, \bar{y}^{k} k$ distinct points of $\partial \Omega$, each one a nondegenerate critical point of $H$ such that $H\left(\bar{y}^{i}\right)>0$. We set

$$
c=\frac{1}{2} \min _{1 \leq i \leq k} H\left(\bar{y}^{i}\right) ; d_{0}=\frac{1}{2} \min _{1 \leq i<j \leq k}\left|\bar{y}^{i}-\bar{y}^{j}\right|
$$

and we consider the function $\widetilde{\widetilde{K}}_{\mu}$ defined on $P_{c, d_{0}}$ as in (3.40-41). In order to show Theorem 1.3 , it is sufficient to prove that for $\mu$ large enough, $\widetilde{\widetilde{K}}_{\mu}$ admits in $P_{c, d_{0}}$ a critical point $y_{\mu}=\left(y_{\mu}^{i}\right)_{1 \leq i \leq k}$ such that for any $i, 1 \leq i \leq k, y_{\mu}^{i} \rightarrow \bar{y}^{i}$ as $\mu \rightarrow+\infty$. (This provides us with a solution $u_{\mu}=\sum_{i=1}^{k} \alpha_{\mu}^{i} U_{\lambda_{\mu}^{i}, y_{\mu}^{i}}+v_{\mu}$ of (4.20) and, according to the argument of the above remark, $u_{\mu}>0$, and is then a solution to (1.1) which concentrates at $\bar{y}^{1}, \cdots, \bar{y}^{k}$ as $\mu \rightarrow+\infty$.) For any $i, 1 \leq i \leq k$, we set, for $y^{i}$ in a neighbourhood of $\bar{y}^{i}$

$$
\xi^{i}=y^{i}-\bar{y}^{i}
$$


and we denote by $\left(\xi_{j}^{i}\right)_{1 \leq j \leq n-1}$ the coordinates of $\xi^{i}$ obtained by projecting $y^{i}$ on the tangent space to $\partial \Omega$ at $\bar{y}^{i}$. Since $\bar{y}^{i}$ is assumed to be a nondegenerate critical point of $H$, it follows from (3.50) that

$$
\frac{\partial \widetilde{\widetilde{K}}_{\mu}}{\partial \tau_{j}^{i}}\left(\bar{y}^{i}\right)=0 \quad 1 \leq i \leq k \quad 1 \leq j \leq n-1
$$

is equivalent to

$$
\xi_{j}^{i}=V_{\mu}^{i, j}(\xi) \quad 1 \leq i \leq k \quad 1 \leq j \leq n-1
$$

where $V_{\mu}^{i, j}$ is a smooth function such that

$$
V_{\mu}^{i, j}(\xi)=0\left[|\xi|^{2}+\left(\frac{(\log \mu)^{4 / 3}}{\mu^{1 / 6}} \quad \text { if } \quad n=6 ; \frac{1}{\mu^{\inf \left(\frac{1}{6}, \frac{2}{n-2}\right)}} \quad \text { if } \quad n \geq 7\right)\right]
$$

Thus, it follows from Brouwer's fixed point theorem that, for $\mu$ large enough, there exists $\xi \in\left(\mathbf{R}^{n-1}\right)^{k}$ such that (4.21) is satisfied, with

$$
\xi_{j}^{i}=0\left(\frac{(\log \mu)^{4 / 3}}{\mu^{1 / 6}} \quad \text { if } \quad n=6 ; \frac{1}{\mu^{\inf \left(\frac{1}{6}, \frac{2}{n-2}\right)}} \quad \text { if } \quad n \geq 7\right)
$$

Hence the result. 


\section{APPENDIX}

\section{A. A minimization result}

In this appendix, we give a proof of Proposition 3.1, adapted from [7]. We recall that, for $k \in \mathbf{N}^{*}, \varepsilon>0$

$$
\begin{array}{r}
B_{\varepsilon}=\left\{(\alpha, \lambda, y) \in \mathbf{R}^{k} \times\left(\mathbf{R}_{+}^{*}\right)^{k} \times(\partial \Omega)^{k} / \frac{\bar{\alpha}}{2}<\alpha_{i}<2 \bar{\alpha}, \lambda_{i}>\frac{1}{\varepsilon}, 1 \leq i \leq k ;\right. \\
\left.\left|y^{i}-y^{j}\right|^{2}>\varepsilon, 1 \leq i<j \leq k\right\}
\end{array}
$$

$V_{\varepsilon}=\left\{u \in H^{1}(\Omega) / \exists(\lambda, y) \in\left(\mathbf{R}_{+}^{*}\right)^{k} \times(\partial \Omega)^{k} /(\bar{\alpha}, \lambda, y) \in B_{\varepsilon},|\nabla(u-\varphi(\bar{\alpha}, \lambda, y))|_{2}<\varepsilon\right\}$

with

$$
\varphi(\alpha, \lambda, y)=\sum_{i=1}^{k} \alpha_{i} U_{\lambda_{i}, y^{i}}
$$

and

$$
\bar{\alpha}=(\bar{\alpha}, \cdots, \bar{\alpha})
$$

We have :

\section{Lemma A1.-}

Let $\left(\varepsilon_{m}\right)$ be a sequence in $\mathbf{R}_{+}^{*}$ such that $\varepsilon_{m}$ goes to zero as $m$ goes to infinity, and $\left(\alpha^{m}, \lambda^{m}, y_{m}\right) \in B_{\varepsilon_{m}},\left(\tilde{\alpha}^{m}, \tilde{\lambda}^{m}, \tilde{y}_{m}\right) \in B_{\varepsilon_{m}}$ such that

$$
\left|\nabla\left(\varphi\left(\alpha^{m}, \lambda^{m}, y_{m}\right)-\varphi\left(\tilde{\alpha}^{m}, \tilde{\lambda}^{m}, \tilde{y}_{m}\right)\right)\right|_{2} \underset{m \rightarrow+\infty}{\longrightarrow} 0
$$

Then (up to permutations on the indices of $\left(\tilde{\alpha}_{i}^{m}, \tilde{\lambda}_{i}^{m}, \tilde{y}_{m}^{i}\right)_{1 \leq i \leq k}$ )

$$
\alpha_{i}^{m}-\tilde{\alpha}_{i}^{m} \rightarrow 0 \quad \frac{\lambda_{i}^{m}}{\tilde{\lambda}_{i}^{m}} \rightarrow 1 \quad \lambda_{i}^{m} \tilde{\lambda}_{i}^{m}\left|y_{m}^{i}-\tilde{y}_{m}^{i}\right|^{2} \rightarrow 0
$$

as $m \rightarrow+\infty, 1 \leq i \leq k$.

\section{Proof of Lemma A1}

Integral estimates show - see Appendix D and [6] - the uniform convergences

$$
\left|\nabla U_{\lambda, y}\right|_{2} \rightarrow \frac{S^{n / 2}}{2(n(n-2))^{\frac{n-2}{2}}} \quad \text { as } \quad \lambda \rightarrow+\infty
$$




$$
\int_{\Omega} \nabla U_{\lambda, y} . \nabla U_{\lambda^{\prime}, y^{\prime}} \rightarrow 0 \quad \text { as } \quad \lambda, \lambda^{\prime}, \frac{\lambda}{\lambda^{\prime}}+\frac{\lambda^{\prime}}{\lambda}+\lambda \lambda^{\prime}\left|y-y^{\prime}\right|^{2} \rightarrow+\infty .
$$

Thus, assumptions of Lemma A1 imply the existence of $C>0$ such that

$$
\forall m \in \mathbf{N}, \forall i, 1 \leq i \leq k, \exists j, 1 \leq j \leq k
$$

such that

$$
\frac{\lambda_{i}^{m}}{\tilde{\lambda}_{j}^{m}}+\frac{\tilde{\lambda}_{j}^{m}}{\lambda_{i}^{m}}+\lambda_{i}^{m} \tilde{\lambda}_{j}^{m}\left|y_{m}^{i}-\tilde{y}_{m}^{j}\right|^{2} \leq C .
$$

Such a $j$ is unique. Indeed, assume that (A.5) holds for another index $\ell \neq j$. We would have

$$
\begin{aligned}
\tilde{\lambda}_{j}^{m} \tilde{\lambda}_{\ell}^{m}\left|\tilde{y}_{m}^{j}-\tilde{y}_{m}^{\ell}\right|^{2} & \leq 2 \tilde{\lambda}_{j}^{m} \tilde{\lambda}_{\ell}^{m}\left(\left|\tilde{y}_{m}^{j}-y_{m}^{i}\right|^{2}+\left|y_{m}^{i}-\tilde{y}_{m}^{\ell}\right|^{2}\right) \\
& \leq 2 \frac{\tilde{\lambda}_{\ell}^{m}}{\lambda_{i}^{m}} \lambda_{i}^{m} \tilde{\lambda}_{j}^{m}\left|y_{m}^{i}-\tilde{y}_{m}^{j}\right|^{2}+2 \frac{\tilde{\lambda}_{j}^{m}}{\lambda_{i}^{m}} \lambda_{i}^{m} \tilde{\lambda}_{\ell}^{m}\left|y_{m}^{i}-\tilde{y}_{m}^{j}\right|^{2} \\
& \leq 4 C^{2}
\end{aligned}
$$

a contradiction for $m$ large enough, since $\left(\tilde{\alpha}^{m}, \tilde{\lambda}_{m}\right) \in B_{\varepsilon_{m}}$ means

$$
\tilde{\lambda}_{j}^{m} \tilde{\lambda}_{\ell}^{m}\left|\tilde{y}_{m}^{j}-\tilde{y}_{m}^{\ell}\right|^{2}>\frac{1}{\varepsilon_{m}}
$$

and $\varepsilon_{m}$ goes to zero as $m$ goes to infinity.

Up to a permutation of the indices, we may assume that $j=i$. With this choice, we note that for $j \neq i$

$$
\left|y_{m}^{i}-\tilde{y}_{m}^{j}\right| \geq\left|\tilde{y}_{m}^{j}-\tilde{y}_{m}^{i}\right|-\left|y_{m}^{i}-\tilde{y}_{m}^{i}\right|>\varepsilon_{m}^{1 / 2}-C^{1 / 2} \varepsilon_{m}>\frac{\varepsilon_{m}^{1 / 2}}{2}
$$

for $m$ large enough, so that

$$
\lambda_{i}^{m} \tilde{\lambda}_{j}^{m}\left|y_{m}^{i}-\tilde{y}_{m}^{j}\right|^{2}>\frac{1}{4 \varepsilon_{m}}
$$

and, according to (A.4)

$$
\int_{\Omega} \nabla U_{\lambda_{i}^{m}, y_{i}^{m}} \cdot \nabla U_{\tilde{\lambda}_{j}^{m}, \tilde{y}_{j}^{m}} \rightarrow 0 \quad \text { as } \quad m \rightarrow+\infty .
$$

Consequently

$$
\left|\nabla\left(\sum_{i=1}^{k}\left(\alpha_{i}^{m} U_{\lambda_{i}^{m}, y_{m}^{i}}-\tilde{\alpha}_{i}^{m} U_{\tilde{\lambda}_{i}^{m}, \tilde{y}_{m}^{i}}\right)\right)\right|_{2}^{2}=\sum_{i=1}^{k}\left|\nabla\left(\alpha_{i}^{m} U_{\lambda_{i}^{m}, y_{m}^{i}}-\tilde{\alpha}_{i}^{m} U_{\tilde{\lambda}_{i}^{m}, \tilde{y}_{m}^{i}}\right)\right|_{2}^{2}+o(1) .
$$


(A.1,3) then imply, for any $i, 1 \leq i \leq k$, that

$$
\alpha_{i}^{m}-\tilde{\alpha}_{i}^{m} \rightarrow 0 \quad \text { as } \quad m \rightarrow+\infty
$$

and

$$
\left|\nabla\left(U_{\lambda_{i}^{m}, y_{m}^{i}}-U_{\tilde{\lambda}_{i}^{m}, \tilde{y}_{m}^{i}}\right)\right|_{2}^{2} \rightarrow 0 \quad \text { as } \quad m \rightarrow+\infty .
$$

From (A.3) we have

$$
\left|\nabla\left(U_{\lambda_{i}^{m}, y_{m}^{i}}-U_{\tilde{\lambda}_{i}^{m}, \tilde{y}_{m}^{i}}\right)\right|_{2}^{2}=S^{n / 2}-2 \int_{\Omega} \nabla U_{\lambda_{i}^{m}, y_{m}^{i}} \cdot \nabla U_{\tilde{\lambda}_{i}^{m}, \tilde{y}_{m}^{i}}+o(1)
$$

and from $(\mathrm{A} .4,6)$ we see that the quantity

$$
\frac{\lambda_{i}^{m}}{\tilde{\lambda}_{i}^{m}}+\frac{\tilde{\lambda}_{i}^{m}}{\lambda_{i}^{m}}+\lambda_{i}^{m} \tilde{\lambda}_{i}^{m}\left|y_{i}^{m}-\tilde{y}_{i}^{m}\right|^{2}
$$

is bounded. Then, up to a subsequence, we may assume that

$$
\frac{\tilde{\lambda}_{i}^{m}}{\lambda_{i}^{m}} \rightarrow \delta \in \mathbf{R}_{+}^{*}, \quad \lambda_{i}^{m}\left(\tilde{y}_{m}^{i}-y_{m}^{i}\right) \rightarrow a \in \mathbf{R}^{n} .
$$

Thus, with a change of variable in the integral

$$
\begin{aligned}
\left|\nabla\left(U_{\lambda_{i}^{m}, y_{m}^{i}}-U_{\tilde{\lambda}_{i}^{m}, \tilde{y}_{m}^{i}}\right)\right|_{2}^{2} & =\int_{\lambda_{i}^{m}\left(\Omega-y_{m}^{i}\right)}\left|\nabla U_{1, o}-\nabla U_{\frac{\dot{\lambda}_{i}^{m}}{\lambda_{i}^{m}, \lambda_{i}^{m}\left(\tilde{y}_{m}^{i}-y_{m}^{i}\right)}}\right|^{2} \\
& =\frac{1}{2} \int_{\mathbf{R}^{n}}\left|\nabla U_{1, o}-\nabla U_{\delta, a}\right|^{2}+o(1)
\end{aligned}
$$

using Lebesgue convergence theorem. It follows from (A.6) that $\delta=1$ and $a=0$, and the proof of Lemma A.1 is complete.

\section{Lemma A.2.-}

There exists $\varepsilon_{0}>0$ such that for any $\varepsilon, 0<\varepsilon<\varepsilon_{0}$, and $u \in V_{\varepsilon}$,

$$
\inf _{(\alpha, \lambda, y) \in B_{4 \varepsilon}}|\nabla(u-\varphi(\alpha, \lambda, y))|_{2}
$$

is achieved in $B_{2 \varepsilon}$ and is not achieved in $B_{4 \varepsilon} \backslash B_{2 \varepsilon}$ 


\section{Proof of Lemma A.2.}

Let us show first that the infimum is achieved in $\bar{B}_{4 \varepsilon}$.

Let $\left(\alpha^{m}, \lambda^{m}, y_{m}\right)$ be a minimizing sequence in $B_{4 \varepsilon}$. Up to a subsequence, we may assume that, for any $i, 1 \leq i \leq k$

$$
\begin{array}{ll}
\alpha_{i}^{m} \rightarrow \alpha_{i}^{0} & \frac{\bar{\alpha}}{2} \leq \alpha_{i}^{0} \leq 2 \bar{\alpha} \\
\lambda_{i}^{m} \rightarrow \lambda_{i}^{0} & \frac{1}{\varepsilon} \leq \lambda_{i}^{0} \leq+\infty \\
y_{m}^{i} \rightarrow y_{0}^{i} \in \partial \Omega
\end{array}
$$

with $\left|y_{0}^{i}-y_{0}^{j}\right|^{2} \geq \varepsilon, 1 \leq i<j \leq k$. The only thing to prove is that $\lambda_{i}^{o}$ is finite. Since $u \in V_{\varepsilon}$, there exists $(\lambda, y)$ such that $(\alpha, \lambda, y) \in B_{\varepsilon}$ and $v \in H^{1}(\Omega),\|v\|<\varepsilon$, such that

$$
u=\varphi(\bar{\alpha}, \lambda, y)+v
$$

and we have

$$
\mid \nabla\left(\varphi(\bar{\alpha}, \lambda, y)+v-\left.\varphi\left(\alpha^{m}, \lambda^{m}, y_{m}\right)\right|_{2}<\varepsilon\right.
$$

Therefore

$$
\left|\nabla\left(\varphi(\bar{\alpha}, \lambda, y)-\varphi\left(\alpha^{m}, \lambda^{m}, y_{m}\right)\right)\right|_{2}<2 \varepsilon
$$

Proceeding as in the proof of the previous lemma, we deduce from this inequality that for $\varepsilon$ small enough, up to a permutation of the indices, $\alpha_{i}^{m}, y_{m}^{i}, \frac{\lambda_{i}^{m}}{\lambda_{i}}$ have to be close to $\alpha_{i}, y^{i}, 1$ respectively. This implies that $\lambda_{i}^{o}$ is finite, and the infimum is achieved in $\bar{B}_{4 \varepsilon}$.

Suppose now, arguing by contradiction, that there exists a sequence $u_{m}$ in $H^{1}(\Omega)$ such that $u_{m} \in V_{\varepsilon_{m}}$ with $\varepsilon_{m}$ going to zero as $m$ goes to infinity, and such that for any $m$ the infimum of $|\nabla(u-\varphi(\alpha, \lambda, y))|_{2},(\alpha, \lambda, y) \in B_{4 \varepsilon_{m}}$, is achieved in $\bar{B}_{4 \varepsilon_{m}} \backslash B_{2 \varepsilon_{m}}$. Writing $u$ as in (A.7) we obtain, similarly to (A.8), a sequence $\left(\lambda^{m}, y_{m}\right)$ such that $\left(\bar{\alpha}, \lambda^{m}, y_{m}\right) \in B_{\varepsilon_{m}},\left(\tilde{\alpha}^{m}, \tilde{\lambda}^{m}, \tilde{y}_{m}\right) \in \bar{B}_{4 \varepsilon_{m}} \backslash B_{2 \varepsilon_{m}}$, and

$$
\mid \nabla\left(\varphi\left(\bar{\alpha}, \lambda^{m}, y_{m}\right)-\left.\varphi\left(\tilde{\alpha}^{m}, \tilde{\lambda}^{m}, \tilde{y}_{m}\right)\right|_{2}=o(1)\right.
$$

Lemma A.1 applies, which gives, up to a permutation of the indices of $\left(\tilde{\alpha}^{m}, \tilde{\lambda}^{m}, \tilde{y}_{m}\right)$

$$
\frac{\lambda_{i}^{m}}{\tilde{\lambda}_{i}^{m}}=1+o(1) \quad 1 \leq i \leq k
$$

$\left(\bar{\alpha}, \lambda^{m}, y_{m}\right) \in B_{\varepsilon_{m}}$ implies that $\lambda_{i}^{m}>\frac{1}{\varepsilon_{m}}$, and $\left(\tilde{\alpha}^{m}, \tilde{\lambda}^{m}, \tilde{y}\right) \in \bar{B}_{4 \varepsilon_{m}} \backslash B_{2 \varepsilon_{m}}$ that $\tilde{\lambda}_{i}^{m} \leq \frac{1}{2 \varepsilon_{m}}$, a contradiction with (A.9). Hence the lemma. 


\section{Proof of Proposition 2.1}

We argue again by contradiction. If the proposition is false, Lemma A.2 ensures the existence of a sequence $\left(\varepsilon_{m}\right)$ in $\mathbf{R}_{+}^{*}$ going to zero, a sequence of functions $u_{m}$ in $V_{\varepsilon_{m}}$, and sequences $\left(\alpha^{m}, \lambda^{m}, y_{m}\right)$ and $\left(\tilde{\alpha}^{m}, \tilde{\lambda}^{m}, \tilde{y}_{m}\right)$ in $B_{2 \varepsilon_{m}}$ such that, for any $m$

$$
\left(\alpha^{m}, \lambda^{m}, y_{m}\right) \neq\left(\tilde{\alpha}^{m}, \tilde{\lambda}^{m}, \tilde{y}_{m}\right)
$$

and

$\inf _{(\alpha, \lambda, y) \in B_{4 \varepsilon_{m}}} \mid \nabla\left(u-\left.\varphi(\alpha, \lambda, y)\right|_{2}=\mid \nabla\left(u-\left.\varphi\left(\alpha^{m}, \lambda^{m}, y_{m}\right)\right|_{2}=\left|\nabla\left(u-\varphi\left(\tilde{\alpha}^{m}, \tilde{\lambda}^{m}, \tilde{y}_{m}\right)\right)\right|_{2}\right.\right.$

As a consequence, for any $i, 1 \leq i \leq k$, and any $m$

$$
\begin{array}{cc}
v_{m}=u_{m}-\varphi\left(\alpha^{m}, \lambda^{m}, y_{m}\right) \in E_{\lambda^{m}, y_{m}} & \left|\nabla v_{m}\right|_{2}<\varepsilon_{m} \\
\tilde{v}_{m}=u_{m}-\varphi\left(\tilde{\alpha}^{m}, \tilde{\lambda}^{m}, \tilde{y}_{m}\right) \in E_{\tilde{\lambda}^{m}, \tilde{y}_{m}} & \left|\nabla \tilde{v}_{m}\right|_{2}<\varepsilon_{m} .
\end{array}
$$

Lemma A.1 implies, up to a permutation of the indices of $\left(\tilde{\alpha}_{m}, \tilde{\lambda}^{m}, \tilde{y}_{m}\right)$

$$
\alpha_{i}^{m}-\tilde{\alpha}_{i}^{m}=o(1) \quad \frac{\lambda_{i}^{m}}{\tilde{\lambda}_{i}^{m}}=1+o(1) \quad \lambda_{i}^{m} \tilde{\lambda}_{i}^{m}\left|y_{m}^{i}-\tilde{y}_{m}^{i}\right|^{2}=o(1)
$$

for any $i, 1 \leq i \leq k$. Setting

$$
a_{i}^{m}=\alpha_{i}^{m}-\tilde{\alpha}_{i}^{m} \quad b_{i}^{m}=\frac{\tilde{\lambda}_{i}^{m}}{\lambda_{i}^{m}}-1 \quad c_{i}^{m}=\tilde{\lambda}_{i}^{m}\left(y_{m}^{i}-\tilde{y}_{m}^{i}\right)
$$

we have

$$
a_{i}^{m}=o(1) \quad b_{i}^{m}=o(1) \quad c_{i}^{m}=o(1)
$$

and our aim is to prove that $a_{i}^{m}=b_{i}^{m}=0, c_{i}^{m}=0$. For sake of simplicity, we omit in the following computations the index $m, U_{i}$ denotes $U_{\lambda_{i}^{m}, y_{m}^{i}}$ and $\widetilde{U}_{i}$ denotes $U_{\tilde{\lambda}_{i}^{m}, \tilde{y}_{m}^{i}}$. We are going to show, successively, that

$$
\begin{aligned}
& a_{i}=o\left(\Sigma_{j}\left(\left|a_{j}\right|+\left|b_{j}\right|+\left|c_{j}\right|\right)\right) \\
& b_{i}=o\left(\Sigma_{j}\left(\left|a_{j}\right|+\left|b_{j}\right|+\left|c_{j}\right|\right)\right) \\
& c_{i}=o\left(\Sigma_{j}\left(\left|a_{j}\right|+\left|b_{j}\right|+\left|c_{j}\right|\right)\right)
\end{aligned}
$$

for any $i, 1 \leq i \leq k$, whence, for $m$ large enough, $a_{i}=b_{i}=c_{i}=0$, a contradiction with (A.10) - from which Proposition 2.1 follows. To make the proof complete, let us show, for example, (A.12). We have

$$
\int_{\Omega} \nabla(\varphi(\alpha, \lambda, y)-\varphi(\tilde{\alpha}, \tilde{\lambda}, \tilde{y})) \cdot \nabla U_{i}=\int_{\Omega} \nabla(\tilde{v}-v) \cdot \nabla U_{i}=\int_{\Omega} \nabla \tilde{v} \cdot \nabla\left(U_{i}-\tilde{U}_{i}\right)
$$


because of (A.11). It is easy to see that, for any $j, 1 \leq j \leq k$

$$
\left|\nabla U_{j}-\nabla \widetilde{U}_{j}\right|=0\left(\left(\left|b_{j}\right|+\left|c_{j}\right|\right)\left|\nabla U_{j}\right|+\left|c_{j}\right| \frac{\lambda_{j}^{\frac{n}{2}}}{\left(1+\lambda_{j}^{2}\left|x-y^{j}\right|^{2}\right)^{\frac{n}{2}}}\right) .
$$

Indeed

$$
\begin{aligned}
\lambda_{j}^{2}\left|x-y^{j}\right|^{2} & =\tilde{\lambda}_{j}^{2} \frac{\lambda_{j}^{2}}{\tilde{\lambda}_{j}^{2}}\left(\left|x-\tilde{y}^{j}\right|^{2}+\left|\tilde{y}^{j}-y^{j 2}\right|+2\left(x-\tilde{y}^{j}\right) \cdot\left(\tilde{y}^{j}-y^{j}\right)\right) \\
& =\left(1+0\left(\left|b_{j}\right|\right)\right)\left(\tilde{\lambda}_{j}\left|x-\tilde{y}^{j}\right|^{2}+c_{j}^{2}+0\left(\left|c_{j}\right| \tilde{\lambda}_{j}\left|x-\tilde{y}^{j}\right|\right)\right)
\end{aligned}
$$

so that

$$
\frac{1+\lambda_{j}^{2}\left|x-y^{j}\right|^{2}}{1+\tilde{\lambda}_{j}\left|x-\tilde{y}^{j}\right|^{2}}=1+0\left(\left|b_{j}\right|+\left|c_{j}\right|\right)
$$

uniformly with respect to $x \in \Omega$. Therefore

$$
\begin{aligned}
\left|\nabla U_{j}-\nabla \widetilde{U}_{j}\right| & =(n-2)\left|\lambda_{j}^{\frac{n+2}{2}} \frac{y^{j}-x}{\left(1+\lambda_{j}^{2}\left|x-y^{j}\right|^{2}\right)^{\frac{n}{2}}}-\tilde{\lambda}_{j}^{\frac{n+2}{2}} \frac{\tilde{y}^{j}-x}{\left(1+\tilde{\lambda}_{j}^{2}\left|x-\tilde{y}^{j}\right|^{2}\right)^{\frac{n}{2}}}\right| \\
& =\frac{(n-2) \lambda_{j}^{\frac{n+2}{2}}}{\left(1+\lambda_{j}^{2}\left|x-y^{j}\right|^{2}\right)^{\frac{n}{2}}}\left|y^{j}-x-\left(\frac{\tilde{\lambda}_{j}}{\lambda_{j}}\right)^{\frac{n+2}{2}}\left(\frac{1+\lambda_{j}^{2}\left|x-y^{j}\right|^{2}}{1+\tilde{\lambda}_{j}^{2}\left|x-\tilde{y}^{j}\right|^{2}}\right)^{\frac{n}{2}}\left(\tilde{y}^{j}-x\right)\right| \\
& =\frac{(n-2) \lambda_{j}^{\frac{n+2}{2}}}{\left(1+\lambda_{j}^{2}\left|x-y^{j}\right|^{2}\right)^{\frac{n}{2}}}\left(0\left(\left|b_{j}\right|+\left|c_{j}\right|\right)\left|y^{j}-x\right|+0\left(\frac{c_{j} \mid}{\lambda_{j}}\right)\right) \\
& =0\left(\left|b_{j}\right|+\left|c_{j}\right|\right)\left|\nabla U_{j}\right|+0\left(\left|c_{j}\right| \frac{\lambda_{j}^{\frac{n}{2}}}{\left(1+\lambda_{j}^{2}\left|x-y^{j}\right|^{2}\right)^{\frac{n}{2}}}\right)
\end{aligned}
$$

hence (A.16). From this we deduce, for $i \neq j$

$$
\begin{aligned}
\int_{\Omega} \nabla\left(U_{j}-\tilde{U}_{j}\right) \cdot \nabla U_{i} & =0\left[\left(\left|b_{j}\right|+\left|c_{j}\right|\right) \int_{\Omega}\left(\left|\nabla U_{j}\right|+\left|c_{j}\right| \frac{\lambda_{j}^{\frac{n}{2}}}{\left(1+\lambda_{j}^{2}\left|x-y^{j}\right|^{2}\right)^{\frac{n}{2}}}\right)\left|\nabla U_{i}\right|\right] \\
& =o\left(\left|b_{j}\right|+\left|c_{j}\right|\right)
\end{aligned}
$$

since, as simple computations show - see the estimates in Appendix D - the integral goes to zero as $m$ goes to infinity. Thus

$$
\int_{\Omega} \nabla\left(\alpha_{j} U_{j}-\tilde{\alpha}_{j} \widetilde{U}_{j}\right) \cdot \nabla U_{i}=o\left(\left|a_{j}\right|+\left|b_{j}\right|+\left|c_{j}\right|\right) \quad \text { for } \quad i \neq j
$$

As we have also, according to $(\mathrm{A} .11,16)$ and straightforward computations

$$
\int_{\Omega} \nabla \tilde{v} \cdot \nabla\left(U_{i}-\tilde{U}_{i}\right)=o\left[\left(\int_{\Omega}\left|\nabla U_{i}-\nabla \tilde{U}_{i}\right|^{2}\right)^{1 / 2}\right]=o\left(\left|b_{i}\right|+\left|c_{i}\right|\right)
$$


(A.15) yields

$$
\int_{\Omega} \nabla\left(\alpha_{i} U_{i}-\tilde{a}_{i} \widetilde{U}_{i}\right) \cdot \nabla U_{i}=o\left(\Sigma_{j}\left(\left|a_{j}\right|+\left|b_{j}\right|+\left|c_{j}\right|\right)\right)
$$

On the other hand, we claim that

$$
\int_{\Omega} \nabla\left(U_{i}-\widetilde{U}_{i}\right) \cdot \nabla U_{i}=o\left(\left|b_{i}\right|+\left|c_{i}\right|\right)
$$

Indeed

$$
\begin{aligned}
& \frac{1}{(n-2)^{2}} \int_{\Omega}\left(\nabla U_{i}-\nabla \widetilde{U}_{i}\right) \cdot \nabla U_{i} \\
& =\int_{\Omega}\left(\frac{\lambda_{i}^{\frac{n+2}{2}}\left(y^{i}-x\right)}{\left(1+\lambda_{i}^{2}\left|y^{i}-x\right|^{2}\right)^{n / 2}}-\frac{\tilde{\lambda}_{i}^{\frac{n+2}{2}}\left(\tilde{y}^{i}-x\right)}{\left(1+\tilde{\lambda}_{i}^{2}\left|\tilde{y}^{i}-x\right|^{2}\right)^{n / 2}}\right) \cdot \frac{\lambda_{i}^{\frac{n+2}{2}}\left(y^{i}-x\right)}{\left(1+\lambda_{i}^{2}\left|y^{i}-x\right|^{2}\right)^{n / 2}} d x \\
& =\int_{\lambda_{i}\left(y^{i}-\Omega\right)}\left(\frac{x}{\left(1+|x|^{2}\right)^{\frac{n}{2}}}-\frac{\left(1+b_{i}\right)^{\frac{n}{2}}\left(\left(1+b_{i}\right) x-c_{i}\right)}{\left(1+\left|\left(1+b_{i}\right) x-c_{i}\right|^{2}\right)^{\frac{n}{2}}}\right) \cdot \frac{x}{\left(1+|x|^{2}\right)^{n / 2}} d x \\
& =\int_{\lambda_{i}\left(y^{i}-\Omega\right)}\left[x-\left(\left(1+\frac{n+2}{2} b_{i}\right) x-c_{i}+n \frac{c_{i} \cdot x-b_{i}|x|^{2}}{1+|x|^{2}} x\right)+0\left(b_{i}^{2}+\left|c_{i}\right|^{2}\right)\right] \cdot \frac{x}{\left(1+|x|^{2}\right)^{n}} d x \\
& =\int_{\lambda_{i}\left(y^{i}-\Omega\right)}\left(b_{i}\left(\frac{n+2}{2}|x|^{2}-\frac{n|x|^{4}}{\left.|+| x\right|^{2}}\right)+c_{i} \cdot x\left(1-\frac{n|x|^{2}}{1+|x|^{2}}\right)+0\left(b_{i}^{2}+\left|c_{i}\right|^{2}\right)\right) \frac{d x}{\left(1+|x|^{2}\right)^{n}} .
\end{aligned}
$$

$\lambda_{i}\left(y^{i}-\Omega\right)$ goes to a half space $P$ of $\mathbf{R}^{n}$ such that $0 \in \partial P$. With a good choice of the coordinates, we may assume that $P=\left\{\left(x^{\prime}, x_{n}\right) \in \mathbf{R}^{n-1} \times \mathbf{R} / x_{n}>0\right\}$. Because of oddness

$$
\int_{P} x_{j}\left(1-\frac{n|x|^{2}}{1+|x|^{2}}\right) \frac{d x}{\left(1+|x|^{2}\right)^{n}}=0 \quad 1 \leq j \leq n-1
$$

and, integrating by parts with respect to $x_{n}$, we have

$$
\int_{p} x_{n} \frac{n|x|^{2}}{1+|x|^{2}} \frac{d x}{\left(1+|x|^{2}\right)^{n}}=\int_{P} \frac{d}{d x_{n}}\left(\frac{1}{\left(1+|x|^{2}\right)^{n}}\right)\left(-\frac{|x|^{2}}{2}\right) d x=\int_{P} \frac{x_{n}}{\left(1+|x|^{2}\right)^{n}} d x .
$$

Still integrating by parts, we have also

$$
\begin{aligned}
\int_{P} \frac{n|x|^{4}}{1+|x|^{2}} \frac{d x}{\left(1+|x|^{2}\right)^{n}} & =\frac{n}{2} \sigma_{n-1} \int_{0}^{+\infty} \frac{r^{n+3} d r}{\left(1+r^{2}\right)^{n+1}} \\
& =\frac{\sigma_{n-1}}{2} \int_{0}^{+\infty} \frac{d}{d r}\left(\frac{1}{\left(1+r^{2}\right)^{n}}\right)\left(-\frac{r^{n+2}}{2}\right) d r \\
& =\frac{n+2}{4} \sigma_{n-1} \int_{0}^{+\infty} \frac{r^{n+1}}{\left(1+r^{2}\right)^{n}} d r \\
& =\frac{n+2}{2} \int_{P} \frac{|x|^{2}}{\left(1+|x|^{2}\right)^{n}} d x
\end{aligned}
$$


Finally, we find

$$
\int_{\Omega}\left(\nabla U_{i}-\nabla \tilde{U}_{i}\right) \cdot \nabla U_{i}=o\left(b_{i}+\left|c_{i}\right|\right)
$$

and (A.19) is proved. Coming back to (A.18), we obtain

$$
\left(\alpha_{i}-\tilde{\alpha}_{i}\right) \int_{\Omega}\left|\nabla U_{i}\right|^{2}=o\left(\Sigma_{j}\left(\left|a_{j}\right|+\left|b_{j}\right|+\left|c_{j}\right|\right)\right)
$$

hence (A.12), using (D.6).

(A.13) and (A.14) may be proven using the same arguments, just changing in (A.15) $U_{i}, \widetilde{U}_{i}$ by $\frac{\partial U_{i}}{\partial \lambda_{i}}, \frac{\partial \widetilde{U}}{\partial \tilde{\lambda}_{i}}$ and $\frac{\partial U_{i}}{\partial y_{i}}, \frac{\partial U_{i}}{\partial \tilde{y}_{i}}$ respectively. The computations are quite similar, and we omit them.

\section{B - THE COERCIVITY OF $Q_{\mu, \alpha, \lambda, y}$}

On

$$
\begin{aligned}
E_{\lambda, y}= & \left\{v \in H^{1}(\Omega) / \int_{\Omega} \nabla v \cdot \nabla U_{i}=\int_{\Omega} \nabla v \cdot \nabla \frac{\partial U_{i}}{\partial \lambda_{i}}=\int_{\Omega} \nabla v \cdot \nabla \frac{\partial U_{i}}{\partial y_{j}^{i}}=0\right. \\
& 1 \leq i \leq k ; 1 \leq j \leq n\}
\end{aligned}
$$

we consider the quadratic form

$$
Q_{\mu, \alpha, \lambda, y}(v)=\frac{1}{2}\left(\int_{\Omega}|\nabla v|^{2}+\mu \int_{\Omega} v^{2}-p \int_{\Omega} \varphi_{\alpha, \lambda, y}^{p-1} v^{2}\right) .
$$

We want to prove that $Q_{\mu, \alpha, \lambda, y}$ is coercive, with a modulus of coercivity independent of $(\mu, \alpha, \lambda, y) \in \mathbf{R}_{+}^{*} \times \mathbf{R}^{k} \times\left(\mathbf{R}_{+}^{*}\right)^{k} \times(\partial \Omega)^{k}$ for

$$
\begin{array}{rl}
\mu_{0}<\mu<\delta_{0} \lambda_{i}^{2} & 1 \leq i \leq k \\
\left|\alpha_{i}-\bar{\alpha}\right|<\eta_{0} & 1 \leq i \leq k \\
\lambda_{i}>\frac{1}{\eta_{0}} & 1 \leq i \leq k \\
\left|y^{i}-y^{j}\right|>d_{0} & 1 \leq i<j \leq k
\end{array}
$$

where $d_{0}>0$ is a given constant, and $\eta_{0}, \mu_{0}, \delta_{0}$ are strictly positive constants which have to be chosen independently of each other. We remark that

$$
\varphi_{\alpha, \lambda, y}^{p-1}=\sum_{i=1}^{k} \alpha_{i}^{p-1} U_{i}^{p-1}+0\left(\sum_{\substack{i, j \\ i \neq j}} U_{i}^{p-2} \inf \left(U_{i}, U_{j}\right)\right)
$$


and, using Hölder's inequality and Sobolev embedding theorem

$$
\int_{\Omega} U_{i}^{p-2} \inf \left(U_{i}, U_{j}\right) v^{2}=0\left[\|v\|\left(\int_{\Omega} \inf \left(U_{i}^{p+1}, U_{i}^{\frac{(p-2)(p+1)}{p-1}} U_{j}^{\frac{p+1}{p-1}}\right)\right)^{\frac{p-1}{p+1}}\right]=o(\|v\|)
$$

as $\lambda_{i}, \lambda_{j} \rightarrow+\infty$, as it follows from (D.47). Therefore, choosing $\eta_{0}$ sufficiently small, and noticing that $p \bar{\alpha}^{p-1}=n(n+2)$, we have actually to study the coercivity on $E_{\lambda, y}$ of the quadratic form

$$
\widetilde{Q}_{\mu, \lambda, y}(v)=\frac{1}{2}\left(\int_{\Omega}|\nabla v|^{2}+\mu \int_{\Omega} v^{2}-n(n+2) \sum_{i=1}^{k} \int_{\Omega} U_{i}^{p-1} v^{2}\right)
$$

In the context of Dirichlet boundary conditions, a similar result has been proved by Bahri [6]. It has been adapted in [4] to our case, for $k=1$. What we need here is a reșult which holds for arbitrary $k$ in $\mathbf{N}^{*}$. For $1 \leq i \leq k$, we define

$$
\Omega_{i}=\Omega \cap B\left(y^{i}, \frac{d_{0}}{2}\right)
$$

We remark that, if $i \neq j, \Omega_{i} \cap \Omega_{j}=\emptyset$. For $v \in H^{1}(\Omega)$, we consider

$$
v_{i}=v \cdot 1_{\Omega_{i}}
$$

where $1_{\Omega_{i}}$ is the characteristic function of $\Omega_{i}$. We set also

$$
\begin{gathered}
E_{i}^{-}=\operatorname{Vect}\left(U_{i} ; \frac{\partial U_{i}}{\partial \lambda_{i}} ; \frac{\partial U_{i}}{\partial y_{j}^{i}}, 1 \leq j \leq n\right) \\
E_{i}^{+}=\left\{w \in H^{1}\left(\Omega_{i}\right) / \int_{\Omega_{i}} \nabla U_{i} \cdot \nabla w=\int_{\Omega_{i}} \nabla \frac{\partial U_{i}}{\partial \lambda_{i}} \cdot \nabla w=\int_{\Omega_{i}} \nabla \frac{\partial U_{i}}{\partial y_{j}^{i}} \cdot \nabla w=0,1 \leq j \leq n\right\} .
\end{gathered}
$$

$H^{1}\left(\Omega_{i}\right)=E_{i}^{-} \oplus E_{i}^{+}$, so that there is a unique way to write

$$
v_{i}=v_{i}^{+}+v_{i}^{-} \quad v_{i}^{+} \in E_{i}^{+}, v_{i}^{-} \in E_{i}^{-}
$$

On one hand, according to [4, Lemma 3.4], we know that there exists $\rho_{i}>0$ such that, for any $v \in H^{1}(\Omega)$

$$
\begin{aligned}
\frac{1}{2}\left(\int_{\Omega_{i}}\left|\nabla v_{i}^{+}\right|^{2}+\mu \int_{\Omega_{i}} v_{i}^{+2}\right. & \left.-n(n+2) \int_{\Omega_{i}} U_{i}^{p-1} v_{i}^{+2}\right) \\
& \geq \rho_{i}\left(\int_{\Omega_{i}}\left|\nabla v_{i}^{+}\right|^{2}+\mu \int_{\Omega_{i}} v_{i}^{+2}\right)
\end{aligned}
$$


provided that $\mu$ and $\lambda_{i}$ are large enough, and $\mu / \lambda_{i}^{2}$ is small enough. On the other hand, let us estimate $v_{i}^{-}$, assuming that $v \in E_{\lambda, y}$. We write

$$
v_{i}^{-}=a_{i} U_{i}+b_{i} \frac{\partial U_{i}}{\partial \lambda_{i}}+\sum_{j=1}^{n} c_{j}^{i} \frac{\partial U_{i}}{\partial y_{j}^{i}}
$$

and, as usual, we are going to evaluate $a_{i}, b_{i}, c_{j}^{i}$, multiplying the gradient of (B.4) by $\nabla U_{i}, \nabla \frac{\partial U_{i}}{\partial \lambda_{i}}, \nabla \frac{\partial U_{i}}{\partial y_{j}^{i}}$ successively, and integrating on $\Omega_{i}$. We obtain a nearly diagonal and invertible linear system whose left hand side is given by

$$
\int_{\Omega_{i}} \nabla v_{i}^{-} \cdot \nabla U_{i}=\int_{\Omega_{i}} \nabla v \cdot \nabla U_{i}=-\int_{\Omega \backslash \Omega_{i}} \nabla v \cdot \nabla U_{i}=0\left(\frac{1}{\lambda_{i}^{\frac{n-2}{2}}} \int_{\Omega}|\nabla v|\right)=0\left(\frac{\|v\|}{\lambda_{i}^{\frac{n-2}{2}}}\right)
$$

and, in the same way

$$
\int_{\Omega_{i}} \nabla v_{i}^{-} \cdot \nabla \frac{\partial U_{i}}{\partial \lambda_{i}}=0\left(\frac{\|v\|}{\lambda_{i}^{\frac{n}{2}}}\right) \quad \int_{\Omega} \nabla v_{i}^{-} \cdot \nabla \frac{\partial U_{i}}{\partial y_{j}^{i}}=0\left(\frac{\|v\|}{\lambda_{i}^{\frac{n-2}{2}}}\right)
$$

Then, using (D.27) to solve the linear system, we find

$$
a_{i}=0\left(\frac{\|v\|}{\lambda_{i}^{\frac{n-2}{2}}}\right) ; b_{i}=0\left(\frac{\|v\|}{\lambda_{i}^{\frac{n-4}{2}}}\right) ; c_{j}^{i}=0\left(\frac{\|v\|}{\lambda_{i}^{\frac{n+2}{2}}}\right)
$$

and, from (B.4) and (D.26) we deduce

$$
\left\|v_{i}^{-}\right\|^{2}=0\left(\frac{1}{\lambda_{i}^{n-2}}\left(1+\frac{\mu}{\lambda_{i}^{2}}\right)\|v\|^{2}\right)
$$

Combining (B.3) and (B.5) we get, for any $v \in E_{\lambda, y}$

$$
\begin{aligned}
& \frac{1}{2}\left(\int_{\Omega_{i}}|\nabla v|^{2}+\mu \int_{\Omega_{i}} v^{2}-n(n+2) \int_{\Omega_{i}} U_{i}^{p-1} v^{2}\right) \\
& \geq \frac{\rho_{i}}{2}\left(\int_{\Omega_{i}}|\nabla v|^{2}+\mu \int_{\Omega_{i}} v^{2}\right)+0\left(\frac{\|v\|^{2}}{\lambda_{i}^{n-2}}\right)
\end{aligned}
$$

provided that $\mu$ and $\lambda_{i}$ are large enough, and $\mu / \lambda_{i}^{2}$ is small enough. We write

$$
\begin{aligned}
\widetilde{Q}_{\mu, \lambda, y}(v) & =\frac{1}{2}\left(\int_{\Omega}|\nabla v|^{2}+\mu \int_{\Omega} v^{2}-n(n+2) \sum_{i=1}^{k} \int_{\Omega} U_{i}^{p-1} v^{2}\right) \\
& =\frac{1}{2}\|v\|^{2}-\frac{1}{2} \sum_{i=1}^{k}\left(\int_{\Omega_{i}}|\nabla v|^{2}+\mu \int_{\Omega_{i}} v^{2}\right)+\frac{1}{2} \sum_{i=1}^{k}\left(\int_{\Omega_{i}}|\nabla v|^{2}+\mu \int_{\Omega_{i}} v^{2}\right. \\
& \left.-n(n+2) \int_{\Omega_{i}} U_{i}^{p-1} v^{2}\right)-\frac{n(n+2)}{2} \sum_{i=1}^{k} \int_{\Omega \backslash \Omega_{i}} U_{i}^{p-1} v^{2} .
\end{aligned}
$$


As we have

$$
\int_{\Omega \backslash \Omega_{i}} U_{i}^{p-1} v^{2}=0\left(\frac{1}{\lambda_{i}^{2}} \int_{\Omega} v^{2}\right)=0\left(\frac{\|v\|^{2}}{\mu \lambda_{i}^{2}}\right)
$$

it follows from (B.6) that, for $\mu, \lambda_{i}$ large enough, $\frac{\mu}{\lambda_{i}^{2}}$ small enough, $1 \leq i \leq k$

$$
\begin{aligned}
\widetilde{Q}_{\mu, \lambda, y}(v) & \geq \frac{1}{2}\left(\int_{\Omega \backslash \cup_{i=1}^{k} \Omega_{i}}|\nabla v|^{2}+\mu \int_{\Omega \backslash \cup_{i=1}^{k} \Omega_{i}} v^{2}\right)+\frac{1}{2} \sum_{i=1}^{k} \frac{\rho_{i}}{2}\left(\int_{\Omega_{i}}|\nabla v|^{2}+\mu \int_{\Omega_{i}} v^{2}\right) \\
& +0\left(\sum_{i=1}^{k}\left(\frac{1}{\lambda_{i}^{n-2}}+\frac{1}{\mu \lambda_{i}^{2}}\right)\|v\|^{2}\right)
\end{aligned}
$$

Consequently, setting $\rho=\frac{1}{2} \min \left(\frac{1}{2} ; \frac{\rho_{i}}{4}, 1 \leq i \leq k\right)$, and choosing in (B.1) $\mu_{0}$ large enough, $\eta_{0}$ and $\delta_{0}$ small enough, we obtain

$$
\widetilde{Q}_{\mu, \lambda, y}(v) \geq \rho\|v\|^{2} \quad \forall v \in E_{\lambda, y}
$$

This concludes the proof of Lemma 3.1.

\section{C- THE DERIVATIVES OF $\mathbf{K}_{\mu}$ WITH RESPECT TO $\alpha, \lambda, \mathrm{y}$}

In this appendix, we prove

\section{Lemma C.1.-}

Let $\mu, \alpha, \lambda, y$ satisfy the assumptions of Proposition 3.1, be such that

$$
\mu<\kappa_{2} \lambda_{i} \quad 1 \leq i \leq k
$$

with $\kappa_{2}$ a strictly positive constant. We have

$$
\begin{aligned}
& \frac{\partial K_{\mu}}{\partial \alpha_{i}}(\alpha, \lambda, y, \bar{v})=-C_{6} \eta_{i}-C_{7} \frac{H\left(y^{i}\right)}{\lambda_{i}} \\
& \quad+0\left[\eta_{i}^{2}+\left(\frac{1}{\lambda^{3 / 2}} \text { if } n=5 ; \frac{(\log \lambda)^{4 / 3}}{\lambda^{2}} \text { if } n=6 ; \frac{1}{\lambda^{2}} \text { if } n \geq 7\right)\right]
\end{aligned}
$$

with

$$
\alpha_{i}=\bar{\alpha}\left(1+\eta_{i}\right)
$$

and

$$
\begin{aligned}
C_{6} & =\frac{2 n}{(n(n-2))^{\frac{n+2}{4}}} S^{n / 2}>0 \\
C_{7} & =\bar{\alpha}\left(C_{1}-\bar{\alpha}^{p-1} C_{4}\right) \\
& =(n(n-2))^{\frac{n-2}{4}}(n-1)(n-2) \sigma_{n-2} \frac{\Gamma\left(\frac{n+1}{2}\right) \Gamma\left(\frac{n-3}{2}\right)}{4 \Gamma(n)}>0 .
\end{aligned}
$$




$$
\begin{aligned}
& \frac{\partial K_{\mu}}{\partial \lambda_{i}}(\alpha, \lambda, y, \bar{v})=C_{8} \frac{H\left(y^{i}\right)}{\lambda_{i}^{2}}-C_{9} \frac{\mu}{\lambda_{i}^{3}} \\
& \quad+0\left[\frac{\left|\eta_{i}\right|}{\lambda_{i}^{2}}+\left(\frac{1}{\lambda^{5 / 2}} \text { if } n=5 ; \frac{(\log \lambda)^{4 / 3}}{\lambda^{3}} \text { if } n=6 ; \frac{1}{\lambda^{3}} \text { if } n \geq 7\right)\right]
\end{aligned}
$$

with

$$
\begin{aligned}
& C_{8}=\frac{\bar{\alpha}^{2}}{2}\left(C_{1}-(n-2)^{2} C_{4}\right)=(n(n-2))^{\frac{n-2}{2}}(n-2)^{2} \sigma_{n-2} \frac{\Gamma\left(\frac{n+1}{2}\right) \Gamma\left(\frac{n-3}{2}\right)}{4 \Gamma(n)}>0 \\
& C_{9}=\frac{\bar{\alpha}^{2}}{2} C_{5}=(n(n-2))^{\frac{n-2}{2}} \sigma_{n-1} \frac{\Gamma\left(\frac{n}{2}\right) \Gamma\left(\frac{n-4}{2}\right)}{8 \Gamma(n-2)}>0 .
\end{aligned}
$$

Lastly,

$$
\frac{\partial K_{\mu}}{\partial \tau_{j}^{i}}(\alpha, \lambda, y, \bar{v})=0\left(\frac{1}{\lambda^{1 / 2}} \text { if } n=5 ; \frac{(\log \lambda)^{1 / 2}}{\lambda} \text { if } n=6 ; \frac{1}{\lambda} \text { if } n \geq 7\right) .
$$

Moreover, if $n \geq 6$, and

$$
\kappa_{3} \lambda_{i}<\mu \quad 1 \leq i \leq k
$$

with $\kappa_{3}$ a strictly positive constant,

$$
\begin{aligned}
\frac{\partial K_{\mu}}{\partial \tau_{j}^{i}}(\alpha, \lambda, y, \bar{v}) & =-\frac{C_{8}}{\lambda_{i}} \frac{\partial H}{\partial \tau_{j}^{i}}\left(y^{i}\right) \\
& +0\left(\frac{(\log \lambda)^{4 / 3}}{\lambda^{7 / 6}} \text { if } n=6 ; \frac{1}{\lambda^{\inf \left(\frac{7}{6}, \frac{n}{n-2}\right)}} \text { if } n \geq 7\right) .
\end{aligned}
$$

As a byproduct, we obtain also

\section{Lemma C.2.-}

Let $A_{i}, B_{i}, C_{i, j}, 1 \leq i \leq k, 1 \leq j \leq n-1$ the coefficients which occur in (3.4). With the same assumptions on $\mu, \alpha, \lambda, y$ as above, we have

$$
\begin{aligned}
A_{i} & =0\left(|\eta|+\frac{1}{\lambda}\right) \\
B_{i} & =0(1) \\
C_{i, j} & =0\left(\frac{1}{\lambda^{5 / 2}} \text { if } n=5 ; \frac{1}{\lambda^{3}} \text { if } n \geq 6\right) .
\end{aligned}
$$




\section{Proof of Lemma C.2}

It is a direct consequence of Lemma C.1. Indeed, considering the linear system in $A_{i}, B_{i}, C_{i, j}$ that we obtain taking $w=U_{i}, \frac{\partial U_{i}}{\partial \lambda_{i}}, \frac{\partial U_{i}}{\partial \tau_{j}^{2}} 1 \leq i \leq k, 1 \leq j \leq n-1$, in (3.20), it follows from the definition of $K_{\mu}$ that the $k(n+1)$ terms on the left hand side are equal to

$$
\begin{gathered}
\frac{\partial K_{\mu}}{\partial v} \cdot U_{i}=\frac{\partial K_{\mu}}{\partial \alpha_{i}} \\
\frac{\partial K_{\mu}}{\partial v} \cdot \frac{\partial U_{i}}{\partial \lambda_{i}}=\frac{1}{\alpha_{i}} \frac{\partial K_{\mu}}{\partial \lambda_{i}} \\
\frac{\partial K_{\mu}}{\partial v} \cdot \frac{\partial U_{i}}{\partial \tau_{j}^{i}}=\frac{1}{\alpha_{i}} \frac{\partial K_{\mu}}{\partial \tau_{j}^{i}} .
\end{gathered}
$$

Using estimates (D.27) to invert the system, estimates of $A_{i}, B_{i}, C_{i, j}$ follow from (C.2,4-5,7). We also remark that from (C.2,4-5) only we derive

$$
\left\{\begin{aligned}
A_{i} & =0\left(|\eta|+\frac{1}{\lambda}\right) \\
B_{i} & =0(1) \\
C_{i, j} & =0\left(\frac{1}{\lambda^{5 / 2}} \text { if } n=5 ; \frac{(\log \lambda)^{4 / 3}}{\lambda^{3}} \text { if } n=6 ; \frac{1}{\lambda^{3}} \text { if } n \geq 6\right) .
\end{aligned}\right.
$$

\section{Proof of Lemma C.1}

According to the definition of $K_{\mu}$, we have

$$
\begin{aligned}
\frac{\partial K_{\mu}}{\partial \alpha_{i}}(\alpha, \lambda, y, \bar{v}) & =\int_{\Omega} \nabla\left(\varphi_{\alpha, \lambda, y}+\bar{v}\right) . \nabla U_{i}+\mu \int_{\Omega}\left(\varphi_{\alpha, \lambda, y}+\bar{v}\right) U_{i} \\
& -\int_{\Omega}\left|\varphi_{\alpha, \lambda, y}+\bar{v}\right|^{p-1}\left(\varphi_{\alpha, \lambda, y}+\bar{v}\right) U_{i}
\end{aligned}
$$

and similar expressions for $\frac{\partial K_{\mu}}{\partial \lambda_{i}}$ and $\frac{\partial K_{\mu}}{\partial y_{j}^{2}}$, replacing $U_{i}$ by $\alpha_{i} \frac{\partial U_{i}}{\partial \lambda_{i}}$ and $\alpha_{i} \frac{\partial U_{i}}{\partial y_{j}^{i}}$ respectively. Thus

$$
\begin{aligned}
\frac{\partial K_{\mu}}{\partial \alpha_{i}} & =\alpha_{i} \int_{\Omega}\left|\nabla U_{i}\right|^{2}+\sum_{\substack{j=1 \\
j \neq i}}^{k} \alpha_{j} \int_{\Omega} \nabla U_{i} . \nabla U_{j}+\mu \alpha_{i} \int_{\Omega} U_{i}^{2}+\mu \sum_{\substack{j=1 \\
j \neq 1}}^{k} \alpha_{j} \int_{\Omega} U_{i} U_{j} \\
& -\alpha_{i}^{p} \int_{\Omega} U_{i}^{p+1}+\mu \int_{\Omega} U_{i} \bar{v}-p \alpha_{i}^{p-1} \int_{\Omega} U_{i}^{p} \bar{v} \\
& +0\left(\sum_{\substack{j=1 \\
j \neq i}}^{k} \int_{\Omega} U_{i}^{p} U_{j}+\sum_{\substack{j=1 \\
j \neq i}}^{k} \int_{\Omega} U_{j}^{p} U_{j}\right)+0\left(\int_{\Omega} U_{i}^{p-1} \bar{v}^{2}\right)+0\left(\int_{\Omega} U_{i}|\bar{v}|^{p} \text { if } p>2\right)
\end{aligned}
$$


since

$$
\begin{aligned}
& \left|\varphi_{\alpha, \lambda, y}+\bar{v}\right|^{p-1}\left(\varphi_{\alpha, \lambda, y}+\bar{v}\right) \\
& =\left|\alpha_{i} U_{i}+\sum_{\substack{j \\
j \neq i}} \alpha_{j} U_{j}+\bar{v}\right|^{p-1}\left(\alpha_{i} U_{i}+\sum_{\substack{j \\
j \neq i}} \alpha_{j} U_{j}+\bar{v}\right) \\
& =\alpha_{i}^{p} U_{i}^{p}+p \alpha_{i} U_{i}^{p-1 p-1}\left(\sum_{\substack{j \\
j \neq i}} \alpha_{j} U_{j}+\bar{v}\right) \\
& +0\left(U_{i}^{p-2}\left(\sum_{\substack{j \\
j \neq i}} U_{j}^{2}+\bar{v}^{2}\right)\right)+0\left(\left(\sum_{\substack{j \\
j \neq i}} U_{j}^{p}+|\bar{v}|^{p}\right) \text { if } p>2\right) .
\end{aligned}
$$

Proceeding as in the proof of Proposition 3.1, we obtain

$$
\begin{aligned}
\mu \int_{\Omega} U_{i} \bar{v} & -p \alpha_{i}^{p-1} \int_{\Omega} U_{i}^{p} \bar{v} \\
=0 & {\left[\|\bar{v}\|\left(\frac{1}{\lambda^{3 / 4}} \text { if } n=5 ; \frac{(\log \lambda)^{2 / 3}}{\lambda} \text { if } n=6 ; \frac{1}{\lambda} \text { if } n \geq 7\right)\right] }
\end{aligned}
$$

and Hölder's inequality, Sobolev embedding theorem and estimate (D.17) give

$$
\int_{\Omega} U_{i}^{p-1} \bar{v}^{2}=0\left(\|\bar{v}\|^{2}\right) \quad \int_{\Omega} U_{i}|\bar{v}|^{p}=0\left(\|\bar{v}\|^{p}\right)
$$

Therefore, (3.5) and (C.1) yield

$$
\begin{gathered}
\mu \int_{\Omega} U_{i} \bar{v}-p \alpha_{i}^{p-1} \int_{\Omega} U_{i}^{p} \bar{v}+0\left(\int_{\Omega} U_{i}^{p-1} \bar{v}^{2}\right)+0\left(\int_{\Omega} U_{i}|\bar{v}|^{p} \text { if } p>2\right) \\
=0\left(\frac{1}{\lambda^{3 / 2}} \text { if } n=5 ; \frac{(\log \lambda)^{4 / 3}}{\lambda^{2}} \text { if } n=6 ; \frac{1}{\lambda^{2}} \text { if } n \geq 7\right) .
\end{gathered}
$$

Thus, using estimates (D.6,17,21,26,31,42) and (C.1), we obtain (C.2).

(C.4) is obtained exactly in the same way, noticing that $\frac{\partial U_{i}}{\partial \lambda_{i}}=0\left(\frac{U_{i}}{\lambda_{i}}\right)$ and using the estimates of Appendix D. The computations are quite similar and we omit them.

It only remains to estimate $\frac{\partial K_{\mu}}{\partial \tau_{j}^{i}}$. Without loss of generality, we may assume, up to a translation and a rotation of the orthonormal referential of $\mathbf{R}^{n}$, that

$$
y^{i}=0 \quad\left(\tau_{j}^{i}\right)_{1 \leq j \leq n-1}=\left(y_{j}^{i}\right)_{1 \leq j \leq n-1},
$$


Because of (C.10), we have

(C.12)

$$
\begin{aligned}
\frac{\partial K_{\mu}}{\partial y_{j}^{i}} & =\alpha_{i}^{2} \int_{\Omega} \nabla U_{i} \cdot \nabla \frac{\partial U_{i}}{\partial y_{j}^{i}}+\sum_{\substack{\ell=1 \\
\ell \neq i}}^{k} \alpha_{i} \alpha_{j} \int_{\Omega} \nabla U_{\ell} \cdot \nabla \frac{\partial U_{i}}{\partial y_{j}^{i}}-\alpha_{i}^{p} \int_{\Omega} U_{i}^{p} \frac{\partial U_{i}}{\partial y_{j}^{i}} \\
& +\mu \alpha_{i}^{2} \int_{\Omega} U_{i} \frac{\partial U_{i}}{\partial y_{j}^{i}}+\mu \sum_{\substack{\ell=1 \\
\ell \neq i}}^{k} \alpha_{i} \alpha_{\ell} \int_{\Omega} U_{\ell} \frac{\partial U_{i}}{\partial y_{j}^{i}}+\mu \alpha_{i} \int_{\Omega} \frac{\partial U_{i}}{\partial y_{j}^{i}} \bar{v} \\
& -p \alpha_{i}^{p} \sum_{\substack{j=1 \\
j \neq i}}^{k} \alpha_{j} \int_{\Omega} U_{i}^{p-1} \frac{\partial U_{i}}{\partial y_{j}^{i}} U_{j}-p \alpha_{i}^{p} \int_{\Omega} U_{i}^{p-1} \frac{\partial U_{i}}{\partial y_{j}^{i}} \bar{v} \\
& +0\left(\int_{\Omega} U_{i}^{p-2}\left|\frac{\partial U_{i}}{\partial y_{j}^{i}}\right|\left(\sum_{\substack{j=1 \\
j \neq i}}^{k} U_{j}^{2}+\bar{v}^{2}\right)\right)+0\left(\int_{\Omega}\left|\frac{\partial U_{i}}{\partial y_{j}^{i}}\right|\left(\sum_{\substack{j=1 \\
j \neq i}}^{k} U_{j}^{p}+|\bar{v}|^{p}\right) \text { if } p>2\right)
\end{aligned}
$$

Proceeding again as in the proof of Proposition 3.1, we deduce from estimates (D.41,50) and (C.1)

$$
\mu \alpha_{i} \int_{\Omega} \frac{\partial U_{i}}{\partial y_{j}^{i}} \bar{v}-p \alpha_{i}^{p} \int_{\Omega} U_{i}^{p-1} \frac{\partial U_{i}}{\partial y_{j}^{i}} \bar{v}=0(\|\bar{v}\|) .
$$

Noticing that $\left|\frac{\partial U_{i}}{\partial y_{j}^{i}}\right|=0\left(\lambda_{i} U_{i}\right)$, Hölder's inequality and (D.17) yield

$$
\int_{\Omega} U_{i}^{p-2}\left|\frac{\partial U_{i}}{\partial y_{j}^{i}}\right| \bar{v}^{2}=0\left(\lambda_{i}\|\bar{v}\|^{2}\right) ; \int_{\Omega}\left|\frac{\partial U_{i}}{\partial y_{j}^{i}} \| \bar{v}\right|^{p}=0\left(\lambda_{i}\|\bar{v}\|^{p}\right) .
$$

Therefore, from estimates (D.8,19,23,26,31,43-45), (3.5) and (C.1), we deduce (C.5).

\section{Proof of (C.7)}

All the equalities of Lemma C.1 are proved, except (C.7), which is the most delicate to obtain. To improve our estimate of $\frac{\partial K_{\mu}}{\partial y_{j}^{2}}$, we inspire ourself of an idea of $[3]$.

We remark that with our choice of coordinates - see (C.10) -, $\Omega$ is nearly symmetric with respect to the $x_{n}$-xaxis in a neighbourhood of $y^{i}=0$. Therefore, there exists $\varphi$, a diffeomorphism close to identity in a neighbourhood of 0 , such that $\varphi(\Omega)$ is symmetric with respect to the n-th axis in this neighborhood. $\frac{\partial U_{i}}{\partial y_{j}^{2}}, 1 \leq j \leq n-1$, being odd with respect to $x_{j}, \frac{\widetilde{\partial U}_{i}}{\partial y_{j}^{i}}=\frac{\partial U_{i}}{\partial y_{j}^{i}} \circ \varphi^{-1}$ is nearly odd with respect to $\mathrm{j}$-th coordinate. At the same time, we split $\tilde{v}=v \circ \varphi^{-1}$ into an even and an odd part with respect to the $\mathrm{j}$-th coordinate, and we are able to show that the odd part is 
smaller than the even one. Therefore, through this transformation and this splitting, we may get improved estimates of integrals where both $\frac{\partial U_{i}}{\partial y_{j}^{i}}$ and $\bar{v}$ occur.

Let us now prove this in details, for $i=1, j=1$ (according to our choice of origin and basis in $\mathbf{R}^{n}, y^{1}=0$ and $\mathbf{R}^{n-1} \times\{0\}$ is the tangent space to $\partial \Omega$ at 0$)$. We take $R>0$, small enough, such that

$$
\Omega \cap B(0, R)=\left\{x=\left(x^{\prime}, x_{n}\right) \in \mathbf{R}^{n-1} \times \mathbf{R} / x \in B(0, R), x_{n}>f\left(x^{\prime}\right)\right\}
$$

with

$$
f\left(x^{\prime}\right)=\sum_{i=1}^{n-1} f_{i} x_{i}^{2}+\sum_{1 \leq i \leq j \leq \ell \leq n-1} g_{i j \ell} x_{i} x_{j} x_{\ell}+0\left(\left|x^{\prime}\right|^{4}\right) .
$$

We set

$$
\left\{\begin{aligned}
\varphi: \mathbf{R}^{n-1} \times \mathbf{R} & \rightarrow \mathbf{R}^{n-1} \times \mathbf{R} \\
\left(x^{\prime}, x_{n}\right) & \mapsto z=\left(z^{\prime}, z_{n}\right)=\left(x^{\prime}, x_{n}-f\left(x^{\prime}\right)\right) .
\end{aligned}\right.
$$

From these definitions, we see that there exists $V$ a neighbourhood of 0 in $\mathbf{R}^{n}$ and $R^{\prime}>0$ such that $\varphi$ induces a diffeomorphism between $V$ and $B\left(0, R^{\prime}\right)$. As a consequence, $\varphi$ induces a diffeomorphism between $W=V \cap \Omega$ and $\varphi(W)=B^{+}=$ $B^{+}\left(0, R^{\prime}\right)$, with

$$
B^{+}\left(0, R^{\prime}\right)=\left\{z=\left(z^{\prime}, z_{n}\right) \in \mathbf{R}^{n-1} \times \mathbf{R} / z \in B\left(0, R^{\prime}\right), z_{n}>0\right\} .
$$

In the following, for $u$ a function defined on $W$, we denote by $\tilde{u}$ the corresponding function defined on $B^{+}$such that

$$
u(x)=\tilde{u}(z) \text { with } \quad z=\varphi(x) .
$$

Let $\zeta \in C^{\infty}\left(\mathbf{R}^{+}, \mathbf{R}\right)$ a smooth function such that

$$
0 \leq \xi \leq 1 ; \zeta(t)=1 \text { if } 0 \leq t \leq \frac{1}{2} ; \zeta(t)=0 \text { if } t \geq 1
$$

We set, for $T>0$, and $z \in \mathbf{R}^{n}$

$$
\tilde{\zeta}_{T}(z)=\zeta(T|z|)
$$

and we notice that if $T$ is large enough, $\operatorname{supp}\left(\tilde{\zeta}_{T}\right) \subset B\left(0, R^{\prime}\right)$. For obvious reasons, we denote by $\zeta_{T}$ the function defined on $\mathbf{R}^{n}$ as

$$
\zeta_{T}(x)=\tilde{\zeta}_{T}(z) \text { if } x \in V ; \zeta_{T}(x)=0 \text { if } x \in \mathbf{R}^{n} \backslash V .
$$


For $T$ large enough, $\zeta_{T}$ satisfies

$$
\zeta_{T}(x)=1 \quad \text { for } \quad|x| \leq \frac{1}{4 T} ; \zeta_{T}(x)=0 \quad \text { for } \quad|x| \geq \frac{2}{T} .
$$

In the following, we assume

$$
T=\lambda_{1}^{q} \quad 0<q \leq \frac{1}{3}
$$

We set

$$
\bar{v}=v_{1}+v_{2}+v_{3}
$$

with

$$
\left\{\begin{array}{l}
v_{1}(x)=\tilde{v}_{1}(z)=\frac{1}{2} \tilde{\zeta}_{T}(z)(\widetilde{\bar{v}}(z)+\widetilde{\bar{v}}(\bar{z})) \\
v_{2}(x)=\tilde{v}_{2}(z)=\frac{1}{2} \tilde{\zeta}_{T}(z)(\widetilde{\bar{v}}(z)-\widetilde{\bar{v}}(\bar{z})) \\
v_{3}(x)=\left(1-\zeta_{T}(x)\right) \bar{v}(x)
\end{array}\right.
$$

and

$$
\bar{z}=\left(-z_{1}, z_{2}, \cdots, z_{n}\right) .
$$

$v_{1}$ and $v_{2}$ are assumed to be zero out of $V$. The crucial point is to get a good estimate of $v_{2}$, which represents the "odd part" of $v$ with respect to $x_{1}$. But before estimating $v_{2}$ itself, we need a convenient estimate of $v_{3}$ in a fixed neighbourhood of 0 . In order to perform the next computations, we remark that

$$
\begin{gathered}
\left|\varphi^{\prime}(x)\right|=\left|\frac{d z}{d x}\right|=1 \quad \text { on } \quad \mathbf{R}^{n} \\
\frac{\partial}{\partial x_{i}}=\frac{\partial}{\partial z_{i}}-\frac{\partial f}{\partial x_{i}} \frac{\partial}{\partial z_{n}}, \quad 0 \leq i \leq n-1 \quad \frac{\partial}{\partial x_{n}}=\frac{\partial}{\partial z_{n}}
\end{gathered}
$$

and

$$
|x|^{2}=|z|^{2}+2 \sum_{i=1}^{n-1} f_{i} z_{i}^{2} z_{n}+0\left(|z|^{4}\right) .
$$

Therefore, for any $\delta \in \mathbf{R}$

$$
\begin{aligned}
\left(1+\lambda^{2}|x|^{2}\right)^{\delta} & =\left(1+\lambda^{2}|z|^{2}\right)^{\delta}+2 \delta \lambda^{2}\left(1+\lambda^{2}|z|^{2}\right)^{\delta-1}\left(\sum_{i=1}^{n-1} f_{i} z_{i}^{2}\right) z_{n} \\
& +0\left(\lambda^{2}\left(1+\lambda^{2}|z|^{2}\right)^{\delta-1}|z|^{4}\right)
\end{aligned}
$$




\section{Estimate of $v_{3}$}

More precisely, what we need is an estimate of $v_{3}$ in $W$. For this purpose, we set

$$
v_{4}=\zeta_{S} v_{3}=\zeta_{S}\left(1-\zeta_{T}\right) \bar{v}
$$

where $S>0$ is a fixed number which is chosen such that

$$
\begin{cases}\zeta_{S}(x)=1 & \forall x \in W \quad\left(\text { i.e. } \tilde{\zeta}_{S}(z)=1 \forall z \in B^{+}, \quad \text { that is } \quad S>\frac{1}{R^{\prime}}\right) \\ \zeta_{S}(x)=0 & \forall x \in W^{\prime}, \quad \text { a neighbourhood of } \quad\left\{y^{2}, \cdots, y^{k}\right\} \quad \text { in } \Omega .\end{cases}
$$

In order to estimate $v_{4}$, we write

$$
v_{4}=a U_{1}+b \frac{\partial U_{1}}{\partial \lambda_{1}}+\sum_{j=1}^{n} c_{j} \frac{\partial U_{1}}{\partial y_{j}^{1}}+v_{4}^{\prime}
$$

with

$$
\int_{\Omega} \nabla U_{1} \cdot \nabla v_{4}^{\prime}=\int_{\Omega} \nabla \frac{\partial U_{1}}{\partial \lambda_{1}} \cdot \nabla v_{4}^{\prime}=\int_{\Omega} \nabla \frac{\partial U_{1}}{\partial y_{j}^{1}} \cdot \nabla v_{4}^{\prime}=0 \quad 1 \leq j \leq n .
$$

Multiplying the gradient of (C.28) by $\nabla U_{1}, \nabla \frac{\partial U_{1}}{\partial \lambda_{1}}, \nabla \frac{\partial U_{1}}{\partial y_{j}^{1}}$ respectively and integrating on $\Omega$, we obtain an invertible linear system in $a, b, c_{j}$ which provides us with an estimate of these coefficients. On the left hand side, we have quantities such that, in view of (C.26)

$$
\begin{aligned}
\int_{\Omega} \nabla U_{1} \cdot \nabla v_{4} & =0\left(\int_{\substack{x \in \Omega \\
\frac{1}{4 T} \leq|x|}}\left|\nabla U_{1}\right|(|\nabla \bar{v}|+T|\bar{v}|)\right) \\
& =0\left(\left(1+\frac{T}{\mu^{1 / 2}}\right)\|\bar{v}\|\left(\int_{\frac{1}{4 T} \leq|x|}\left|\nabla U_{1}\right|^{2}\right)^{1 / 2}\right) .
\end{aligned}
$$

The integrals $\int_{\Omega} \nabla \frac{\partial U_{1}}{\partial \lambda_{i}} \cdot \nabla v_{4}$ and $\int_{\Omega} \nabla \frac{\partial U_{1}}{\partial y_{j}^{1}} . \nabla v_{4}$ may be treated in the same way, and, using (C.6,19) and estimates (D. 29) we get

$$
\left\{\begin{array}{l}
\int_{\Omega} \nabla U_{1} \cdot \nabla v_{4}=0\left(\frac{T^{\frac{n-2}{2}}}{\lambda_{1}^{\frac{n-2}{2}}}\|\bar{v}\|\right) \\
\int_{\Omega} \nabla \frac{\partial U_{1}}{\partial \lambda_{1}} \cdot \nabla v_{4}=0\left(\frac{T^{\frac{n-2}{2}}}{\lambda_{1}^{\frac{n}{2}}}\|\bar{v}\|\right) \\
\int_{\Omega} \nabla \frac{\partial U_{1}}{\partial y_{j}^{1}} \cdot \nabla v_{4}=0\left(\frac{T^{\frac{n}{2}}}{\lambda_{1}^{\frac{n-2}{2}}}\|\bar{v}\|\right) \quad 1 \leq j \leq n .
\end{array}\right.
$$


Therefore, through (D.27), the linear system yields

$$
a=0\left(\frac{T^{\frac{n-2}{2}}}{\lambda_{1}^{\frac{n-2}{2}}}\|\bar{v}\|\right) ; b=0\left(\frac{T^{\frac{n-2}{2}}}{\lambda_{1}^{\frac{n-4}{2}}}\|\bar{v}\|\right) ; c_{j}=0\left(\frac{T^{\frac{n}{2}}}{\lambda_{1}^{\frac{n+2}{2}}}\|\bar{v}\|\right)
$$

In order to estimate $v_{4}^{\prime}$, we take $w=v_{4}^{\prime}$ in (3.20). On the right hand side we find

$$
\mathcal{R}=\sum_{i=1}^{k}\left(A_{i} \int_{\Omega} \nabla U_{i} \cdot \nabla v_{4}^{\prime}+B_{i} \int_{\Omega} \nabla \frac{\partial U_{i}}{\partial \lambda_{i}} \cdot \nabla v_{4}^{\prime}+\sum_{j=1}^{n-1} C_{i j} \int_{\Omega} \nabla \frac{\partial U_{i}}{\tau_{j}^{i}} . \nabla v_{4}^{\prime}\right) .
$$

From $($ C.28, 29) we deduce

$$
\begin{aligned}
\mathcal{R} & =\sum_{i=2}^{k}\left(A_{i} \int_{\Omega} \nabla U_{i} \cdot \nabla\left(v_{4}-a U_{1}-b \frac{\partial U_{i}}{\partial \lambda_{1}}-\sum_{j=1}^{n} c_{j} \frac{\partial U_{1}}{\partial y_{j}^{1}}\right) .\right. \\
& +B_{i} \int_{\Omega} \nabla \frac{\partial U_{i}}{\partial \lambda_{i}} \cdot \nabla\left(v_{4}-a U_{1}-b \frac{\partial U_{1}}{\partial \lambda_{1}}-\sum_{j=1}^{n} c_{j} \frac{\partial U_{1}}{\partial y_{j}^{1}}\right) \\
& \left.+\sum_{\ell=1}^{n-1} C_{i, \ell} \int_{\Omega} \nabla \frac{\partial U}{\partial \tau_{\ell}^{i}} \cdot \nabla\left(v_{4}-a U_{1}-b \frac{\partial U_{1}}{\partial \lambda_{i}}-\sum_{j=1}^{n} c_{j} \frac{\partial U_{1}}{\partial y_{j}^{1}}\right)\right) .
\end{aligned}
$$

We note that, for $2 \leq i \leq k$, since $\zeta_{S}$ is identically zero in a neighbourhood of $y^{i}$

$$
\int_{\Omega} \nabla U_{i} \cdot \nabla v_{4}=\int_{\Omega} \nabla U_{i} \cdot \nabla\left(\zeta_{S}\left(1-\zeta_{T}\right) \bar{v}\right)=0\left(\frac{1}{\lambda_{i}^{\frac{n-2}{2}}} \int_{\Omega}(|\nabla \bar{v}|+T|\bar{v}|)\right.
$$

and similar expressions for $\int_{\Omega} \nabla \frac{\partial U_{i}}{\partial \lambda_{i}} \cdot \nabla v_{4}$ and $\int_{\Omega} \nabla \frac{\partial U_{i}}{\partial \tau_{j}^{i}} . \nabla v_{4}$. In view of (C.19) and (C.6), we find

$$
\left\{\begin{array}{l}
\int_{\Omega} \nabla U_{i} \cdot \nabla v_{4}=0\left(\frac{\|\bar{v}\|}{\lambda_{i}^{\frac{n-2}{2}}}\right) \\
\int_{\Omega} \nabla \frac{\partial U_{i}}{\partial \lambda_{i}} \cdot \nabla v_{4}=0\left(\frac{\|v\|}{\lambda_{i}^{\frac{n}{2}}}\right) \\
\int_{\Omega} \nabla \frac{\partial U_{i}}{\partial \tau_{j}^{i}} \cdot \nabla v_{4}=0\left(\frac{\|\bar{v}\|}{\lambda_{i}^{\frac{n-2}{2}}}\right) \\
2 \leq i \leq k, 1 \leq j \leq n-1 .
\end{array}\right.
$$

This, together with (C.8,19,31) and (D.26), leads to

$$
\mathcal{R}=0\left[\left(\frac{|\eta|}{\lambda^{\frac{n-2}{2}}}+\frac{1}{\lambda^{\frac{n}{2}}}\right)\|\bar{v}\|\right]
$$


The left hand side is equal to (C.34)

$$
\mathcal{L}=\int_{\Omega} \nabla\left(\varphi_{\alpha, \lambda, y}+\bar{v}\right) \cdot \nabla v_{4}^{\prime}+\mu \int_{\Omega}\left(\varphi_{\alpha, \lambda, y}+\bar{v}\right) v_{4}^{\prime}-\int_{\Omega}\left|\varphi_{\alpha, \lambda, y}+\bar{v}\right|^{p-1}\left(\varphi_{\alpha, \lambda, y}+\bar{v}\right) v_{4}^{\prime} .
$$

Let us compute the first integral

$$
\begin{aligned}
& \int_{\Omega} \nabla\left(\varphi_{\alpha, \lambda, y}+\bar{v}\right) \cdot \nabla v_{4}^{\prime} \\
& =\sum_{i=1}^{k} \alpha_{i} \int_{\Omega} \nabla U_{i} \cdot \nabla v_{4}^{\prime}+\int_{\Omega} \nabla \bar{v} \cdot \nabla v_{4}^{\prime} \\
& =\sum_{i=2}^{k} \alpha_{i} \int_{\Omega} U_{i} \cdot \nabla\left(v_{4}-a U_{1}-b^{\prime} \frac{\partial U_{1}}{\partial \lambda_{1}}-\sum_{j=1}^{n} c_{j} \frac{\partial U_{1}}{\partial y_{j}^{1}}\right) \\
& \quad+\int_{\Omega} \nabla\left(v_{1}+v_{2}+\left(1-\zeta_{S}\right) v_{3}+v_{4}^{\prime}\right) \cdot \nabla v_{4}^{\prime} \\
& \quad \sum_{i=2}^{k} \alpha_{i}\left(\int_{\Omega} \nabla U_{i} \cdot \nabla v_{4}-a \int_{\Omega} \nabla U_{i} \cdot \nabla U_{1}-b \int_{\Omega} \nabla U_{i} \cdot \nabla \frac{\partial U_{1}}{\partial \lambda_{1}}-\sum_{j=1}^{n} c_{j} \int_{\Omega} \nabla U_{i} \cdot \nabla \frac{\partial U_{1}}{\partial y_{j}^{1}}\right) \\
& \quad+\int_{\Omega}\left|\nabla v_{4}^{\prime}\right|^{2}+\int_{\Omega} \nabla\left(v_{1}+v_{2}+\left(1-\zeta_{S}\right) v_{3}\right) \cdot \nabla v_{4} \\
& \quad-\int_{\Omega} \nabla\left(\bar{v}-v_{4}\right) \cdot \nabla\left(a U_{1}+b \frac{\partial U_{1}}{\partial \lambda_{1}}+\sum_{j=1}^{n} c_{j} \frac{\partial U_{1}}{\partial y_{j}^{\prime}}\right) \\
& =\int_{\Omega}\left|\nabla v_{4}^{\prime}\right|^{2}+\int_{\Omega} \nabla\left(v_{1}+v_{2}+\left(1-\zeta_{S}\right) v_{3}\right) \nabla\left(\zeta_{S} v_{3}\right)+0\left(\frac{\|\bar{v}\|}{\lambda^{\frac{n-2}{2}}}\right)
\end{aligned}
$$

using (C.30-32) and (D.26). Moreover, we have

$$
\begin{aligned}
& \int_{\Omega} \nabla\left(v_{1}+v_{2}+\left(1-\zeta_{S}\right) v_{3}\right) \nabla\left(\zeta_{S} v_{3}\right) \\
& =\int_{\Omega} \nabla\left(v_{1}+v_{2}\right) \cdot \nabla v_{3}+\int_{\Omega} \nabla\left(\left(1-\zeta_{S}\right) v_{3}\right) \cdot \nabla\left(\zeta_{S} v_{3}\right) \\
& =\int_{\Omega} \nabla\left(\zeta_{T} \bar{v}\right) \cdot \nabla\left(\left(1-\zeta_{T}\right) \bar{v}\right)+\int_{\Omega} \nabla\left(\left(1-\zeta_{S}\right) v_{3}\right) \nabla\left(\zeta_{S} v_{3}\right) \\
& =\int_{\Omega} \zeta_{T}\left(1-\zeta_{T}\right)|\nabla \bar{v}|^{2}+\int_{\Omega}\left(1-2 \zeta_{T}\right) \nabla \zeta_{T} \cdot \bar{v} \nabla \bar{v}-\int_{\Omega}\left|\nabla \zeta_{T}\right|^{2}|\bar{v}|^{2} \\
& +\int_{\Omega} \zeta_{S}\left(1-\zeta_{S}\right)\left|\nabla v_{3}\right|^{2}+\int_{\Omega}\left(1-2 \zeta_{S}\right) \nabla \zeta_{S} \cdot v_{3} \nabla v_{3}-\int_{\Omega}\left|\nabla \zeta_{S}\right|^{2} v_{3}^{2} \\
& \geq 0\left(T \int _ { \Omega } \left|\bar{v}\left\|\left.\nabla \bar{v}\left|+T^{2} \int_{\Omega}\right| \bar{v}\right|^{2}+\int_{\Omega}\left|v_{3} \| \nabla v_{3}\right|+\int_{\Omega} v_{3}^{2}\right)\right.\right. \\
& \geq 0\left(\frac{T}{\lambda^{1 / 2}}\|\bar{v}\|^{2}\right)
\end{aligned}
$$


using Schwarz inequality, (C.19) and (C.6). Therefore

$$
\int_{\Omega} \nabla\left(\varphi_{\alpha, \lambda, y}+\bar{v}\right) \cdot \nabla v_{4}^{\prime} \geq \int_{\Omega}\left|\nabla v_{4}^{\prime}\right|^{2}+0\left(\frac{T}{\lambda^{1 / 2}}\|\bar{v}\|^{2}+\frac{\|\bar{v}\|}{\lambda^{\frac{n-2}{2}}}\right) .
$$

Let us consider now the second integral in (C.34). According to (C.28)

$$
\begin{aligned}
& \mu \int_{\Omega}\left(\varphi_{\alpha, \lambda, y}+\bar{v}\right) v_{4}^{\prime} \\
& =\mu \sum_{i=1}^{k} \alpha_{i} \int_{\Omega} U_{i}\left(v_{4}-a U_{1}-b \frac{\partial U_{1}}{\partial \lambda_{1}}-\sum_{j=1}^{n} c_{j} \frac{\partial U_{1}}{\partial y_{j}^{1}}\right)+\mu \int_{\Omega} v_{4}^{\prime 2} \\
& +\mu \int_{\Omega}\left(v_{1}+v_{2}+\left(1-\zeta_{S}\right) v_{3}+a U_{1}+b \frac{\partial U_{1}}{\partial \lambda_{1}}+\sum_{j=1}^{n} c_{j} \frac{\partial U_{1}}{\partial y_{j}^{1}}\right) \\
& .\left(\zeta_{S} v_{3}-a U_{1}-b \frac{\partial U_{1}}{\partial \lambda_{1}}-\sum_{j=1}^{n} c_{j} \frac{\partial U_{1}}{\partial y_{j}^{1}}\right) .
\end{aligned}
$$

We have

$$
\begin{aligned}
\int_{\Omega} U_{1} v_{4} & =\int_{\Omega} \zeta_{S}\left(1-\zeta_{T}\right) U_{1} \bar{v} \\
& =0\left(\int_{\frac{1}{2 T} \leq|x|} U_{1}|\bar{v}|\right) \\
& =0\left(\left(\int_{\Omega} \bar{v}^{2}\right)^{1 / 2}\left(\int_{\frac{1}{4 T} \leq|x|} U_{1}^{2}\right)^{1 / 2}\right)
\end{aligned}
$$

hence

$$
\int_{\Omega} U_{1} v_{4}=0\left(\frac{T^{\frac{n-4}{2}}}{\lambda^{\frac{n-1}{2}}}\|\bar{v}\|\right)
$$

because of (D.25) and (C.6), and, for $2 \leq i \leq k$

$$
\int_{\Omega} U_{i} v_{4}=0\left(\frac{1}{\lambda_{i}^{\frac{n-2}{2}}}\left(\int_{\Omega}|\bar{v}|^{2}\right)^{1 / 2}\right)=0\left(\frac{1}{\lambda^{\frac{n-1}{2}}}\|\bar{v}\|\right)
$$

because of (C.6). Therefore, using (C.1,31) and (D.21-23, 31)

$$
\mu \sum_{i=1}^{n} \alpha_{i} \int_{\Omega} U_{i}\left(v_{4}-a U_{1}-b \frac{\partial U_{1}}{\partial \lambda_{1}}-\sum_{j=1}^{n} c_{j} \frac{\partial U_{1}}{\partial y_{j}^{1}}\right)=0\left(\frac{T^{\frac{n-4}{2}}}{\lambda^{\frac{n-3}{2}}}\right) .
$$


Lastly

$$
\begin{aligned}
& \mu \int_{\Omega}\left(v_{1}+v_{2}+\left(1-\zeta_{S}\right) v_{3}+a U_{1}+b \frac{\partial U_{1}}{\partial \lambda_{1}}+\sum_{j=1}^{n} c_{j} \frac{\partial U_{1}}{\partial y_{j}^{\prime}}\right)\left(\zeta_{S} v_{3}-a U_{1}-b \frac{\partial U_{1}}{\partial \lambda_{1}}-\sum_{j=1}^{n} c_{j} \frac{\partial U_{1}}{\partial y_{j}^{1}}\right) \\
& =\mu \int_{\Omega}\left(\zeta_{T}+\left(1-\zeta_{S}\right)\left(1-\zeta_{T}\right)\right) \zeta_{S}\left(1-\zeta_{T}\right) \bar{v}^{2}-\mu \int_{\Omega}\left(a U_{1}+b \frac{\partial U_{1}}{\partial \lambda_{1}}+\sum_{j=1}^{n} c_{j} \frac{\partial U_{1}}{\partial y_{j}^{1}}\right)^{2} \\
& -\mu \int_{\Omega}\left(a U_{1}+b \frac{\partial U_{1}}{\partial \lambda_{1}}+\sum_{j=1}^{n} c_{j} \frac{\partial U_{1}}{\partial y_{j}^{1}}\right)\left(v_{1}+v_{2}+\left(1-2 \zeta_{S}\right) v_{3}\right) \\
& \geq 0\left[\mu \int_{\Omega}\left(a^{2} U_{1}^{2}+b^{2}\left(\frac{\partial U_{1}}{\partial \lambda_{1}}\right)^{2}+\sum_{j=1}^{n} c_{j}^{2}\left(\frac{\partial U_{1}}{\partial y_{j}^{\prime}}\right)^{2}\right)+\mu \int_{\Omega}\left|a U_{1}+b \frac{\partial U_{1}}{\partial \lambda_{1}}+\sum_{j=1}^{n} c_{j} \frac{\partial U_{1}}{\partial y_{j}^{\prime}}\right||\bar{v}|\right] \\
& \geq 0\left(\frac{T^{\frac{n-2}{2}}}{\lambda^{\frac{n-2}{2}}}\|\bar{v}\|^{2}\right)
\end{aligned}
$$

because of (C.1,6,19,31) (D.31) and, still using (C.6) (D.31)

$$
\begin{aligned}
& \int_{\Omega} U_{1}|\bar{v}|=0\left[\left(\int_{\Omega} \bar{v}^{2}\right)^{1 / 2}\left(\int_{\Omega} U_{1}^{2}\right)^{1 / 2}\right]=0\left(\frac{1}{\lambda^{3 / 2}}\|\bar{v}\|\right) \\
& \int_{\Omega}\left|\frac{\partial U_{1}}{\partial \lambda_{1}} \| \bar{v}\right|=0\left(\frac{1}{\lambda^{5 / 2}}\|\bar{v}\|\right) \\
& \int_{\Omega}\left|\frac{\partial U_{1}}{\partial y_{j}^{1}} \| \bar{v}\right|=0\left(\frac{1}{\lambda^{1 / 2}}\|\bar{v}\|\right) .
\end{aligned}
$$

Thus

$$
\mu \int_{\Omega}\left(\varphi_{\alpha, \lambda, y}+\bar{v}\right) v_{4}^{\prime} \geq \mu \int_{\Omega} v_{4}^{\prime 2}+0\left(\frac{T^{\frac{n-4}{2}}}{\lambda^{\frac{n-2}{2}}}\|\bar{v}\|\right) .
$$

The third integral in (C.34) writes (note that for $n \geq 6, p \leq 2$ )

$$
\begin{aligned}
& \int_{\Omega}\left|\varphi_{\alpha, \lambda, y}+\bar{v}\right|^{p-1}\left(\varphi_{\alpha, \lambda, y}+\bar{v}\right) v_{4}^{\prime} \\
& =\int_{\Omega}\left(\left(\alpha_{1} U_{1}\right)^{p}+p\left(\alpha_{1} U_{1}\right)^{p-1}\left(\sum_{j=2}^{k} \alpha_{j} U_{j}+\bar{v}\right)+0\left(\sum_{j=2}^{k} U_{j}^{p}+|\bar{v}|^{p}\right)\right) v_{4}^{\prime} \\
& =\int_{\Omega}\left(p \alpha_{1}^{p-1} U_{1}^{p-1} \sum_{j=2}^{k} \alpha_{j} U_{j}+0\left(\sum_{j=2}^{n} U_{j}^{p}+|\bar{v}|^{p}\right)\right)\left(v_{4}-a U_{1}-b \frac{\partial U_{1}}{\partial \lambda_{1}}-\sum_{j=1}^{n} c_{j} \frac{\partial U_{1}}{\partial y_{j}^{1}}\right) \\
& +\alpha_{1}^{p} \int_{\Omega} U_{1}^{p} v_{4}^{\prime}+p \alpha_{1}^{p-1} \int_{\Omega} U_{1}^{p-1} v_{4}^{\prime 2}+p \alpha_{1}^{p-1} \int_{\Omega} U_{1}^{p-1}\left(\bar{v}-v_{4}^{\prime}\right) v_{4}^{\prime} .
\end{aligned}
$$


We have, for $2 \leq j \leq k$, using Hölder's inequality, Sobolev embedding theorem and (D.38)

$$
\begin{aligned}
\int_{\Omega} U_{1}^{p-1} U_{j} v_{4} & =0\left[\frac{\|\bar{v}\|}{\lambda_{j}^{\frac{n-2}{2}}}\left(\int_{\Omega} U_{1}^{\frac{(p-1)(p+1)}{p}}\right)^{\frac{p}{p+1}}\right] \\
& =0\left[\|\bar{v}\|\left(\frac{(\log \lambda)^{2 / 3}}{\lambda^{4}} \text { if } n=6 ; \frac{1}{\lambda^{\frac{n+2}{2}}} \text { if } n \geq 7\right)\right] \\
\int_{\Omega} U_{j}^{p}\left|v_{4}\right| & =0\left(\frac{1}{\lambda_{j}^{\frac{n+2}{2}}} \int_{\Omega}|\bar{v}|\right)=0\left(\frac{1}{\lambda^{\frac{n+3}{2}}}\|\bar{v}\|\right)
\end{aligned}
$$

because of (C.6). Since we have also, using Hölder's inequality, (D.17), and noticing that $\frac{\partial U_{i}}{\partial \lambda_{i}}=0\left(\frac{U_{i}}{\lambda_{i}}\right), \frac{\partial U_{i}}{\partial y_{j}^{i}}=0\left(\lambda_{i} U_{i}\right)$

$(C .38) \quad \int_{\Omega} U_{1}|\bar{v}|^{p}=0\left(\|\bar{v}\|^{p}\right) ; \int_{\Omega}\left|\frac{\partial U_{1}}{\partial \lambda_{1}}\left\|\left.\bar{v}\right|^{p}=0\left(\frac{\|\bar{v}\|^{p}}{\lambda_{1}}\right) ; \int_{\Omega}\left|\frac{\partial U_{1}}{\partial y_{j}^{1}} \| \bar{v}\right|^{p}=0\left(\lambda_{1}\|\bar{v}\|^{p}\right)\right.\right.$

(D.42), (C.19,31) and (3.24) lead to

$$
\begin{aligned}
& \int_{\Omega}\left(p \alpha_{1}^{p-1} U_{1}^{p-1} \sum_{j=2}^{k} \alpha_{j} U_{j}+0\left(\sum_{j=2}^{k} U_{j}^{p}+|\bar{v}|^{p}\right)\right)\left(v_{4}-a U_{1}-b \frac{\partial U_{1}}{\partial \lambda_{1}}-\sum_{j=1}^{n} c_{j} \frac{\partial U_{1}}{\partial y_{j}^{1}}\right) \\
& =0\left(\|\bar{v}\|\left(\frac{(\log \lambda)^{4 / 3}}{\lambda^{2}} \text { if } n=6 ; \frac{1}{\lambda^{\frac{n+2}{n-2}}} \text { if } n>6\right)\right) .
\end{aligned}
$$

We have also

$$
\begin{aligned}
\int_{\Omega} U_{1}^{p} v_{4}^{\prime} & =-\frac{1}{n(n-2)} \int_{\Omega} \Delta U_{1} \cdot v_{4}^{\prime} \\
& =-\frac{1}{n(n-2)} \int_{\partial \Omega} \frac{\partial U_{1}}{\partial \nu} v_{4}^{\prime} \quad \text { integrating by parts } \\
& =-\frac{1}{n(n-2)} \int_{\partial \Omega} \frac{\partial U_{1}}{\partial \nu}\left(v_{4}-a U_{1}-b \frac{\partial U_{1}}{\partial \lambda_{1}}-\sum_{j=1}^{n} c_{j} \frac{\partial U_{1}}{\partial y_{j}^{1}}\right) \\
& =0\left(\|\bar{v}\|\left(\int_{\frac{\partial \Omega}{4 T \leq|x|}}\left|\frac{\partial U_{1}}{\partial \nu}\right|^{\frac{2(n-1)}{n}}\right)^{\frac{n}{2(n-1)}}+\int_{\partial \Omega}\left|\frac{\partial U_{1}}{\partial \nu} \| a U_{1}+b \frac{\partial U_{1}}{\partial \lambda_{1}}+\sum_{j=1}^{n} c_{j} \frac{\partial U_{1}}{\partial y_{j}}\right|\right) .
\end{aligned}
$$

Using (C.19,31) and (D.51,56), we get

$$
\int_{\Omega} U_{1}^{p} v_{4}^{\prime}=0\left(\frac{T^{\frac{n-4}{2}}}{\lambda_{1}^{\frac{n-2}{2}}}\|\bar{v}\|\right) .
$$


Lastly

$$
\begin{aligned}
& \int_{\Omega} U_{1}^{p-1}\left(\bar{v}-v_{4}^{\prime}\right) v_{4}^{\prime} \\
& =\int_{\Omega} U_{1}^{p-1}\left(\bar{v}-v_{4}+a U_{1}+b \frac{\partial U_{1}}{\partial \lambda_{1}}+\sum_{j=1}^{n} c_{j} \frac{\partial U_{1}}{\partial y_{j}^{1}}\right)\left(v_{4}-a U_{1}-b \frac{\partial U_{1}}{\partial \lambda_{1}}-\sum_{j=1}^{n} c_{j} \frac{\partial U_{1}}{\partial y_{j}^{1}}\right) \\
& =\int_{\Omega} U_{1}^{p-1}\left(\left(1-\left(1-\zeta_{T}\right) \zeta_{S}\right) \bar{v}+a U_{1}+b \frac{\partial U_{1}}{\partial \lambda_{1}}+\sum_{j=1}^{n} c_{j} \frac{\partial U_{1}}{\partial y_{j}^{1}}\right) \\
& \qquad \int_{\Omega} U_{1}^{p-1}\left(|\bar{v}|\left|a U_{1}+b \frac{\partial U_{1}}{\partial \lambda_{1}}+\sum_{j=1}^{n} c_{j} \frac{\partial U_{1}}{\partial y_{j}^{1}}\right|+a^{2} U_{1}^{2}+b^{2}\left(\frac{\partial U_{1}}{\partial \lambda_{1}}\right)^{2}+\sum_{j=1}^{n} c_{j}^{2}\left(\frac{\partial U_{1}}{\partial y_{j}^{1}}\right)^{2}\right) \\
& =0 \\
& \left.+\int_{|x| \geq \frac{1}{4 T}} U_{1}^{p-1} \bar{v}^{2}\right] .
\end{aligned}
$$

Using (D.2,17), and the fact that, in view of (C.6)

$$
\int_{\substack{|x| \geq \frac{1}{4 T} \\ \Omega}} U_{1}^{p-1} \bar{v}^{2}=0\left(\frac{T^{4}}{\lambda^{2}} \int_{\Omega}|\bar{v}|^{2}\right)=0\left(\frac{T^{4}}{\lambda^{3}}\|\bar{v}\|^{2}\right)
$$

we get, because of Hölder's and Sobolev inequalities, (C.19) and (C.31)

$$
\begin{aligned}
& \int_{\Omega} U_{1}^{p-1}\left(\bar{v}-v_{4}^{\prime}\right) v_{4}^{\prime} \\
& =0\left[\|\bar{v}\|\left(|a|+\frac{|b|}{\lambda_{1}}+\sum_{j=1}^{n} \lambda_{1}\left|c_{j}\right|\right)+a^{2}+\frac{b^{2}}{\lambda_{1}^{2}}+\lambda_{1}^{2} \sum_{j=1}^{n} c_{j}^{2}+\frac{T^{4}}{\lambda^{3}}\|\bar{v}\|^{2}\right] \\
& =0\left(\left(\frac{T^{\frac{n-2}{2}}}{\lambda^{\frac{n-2}{2}}}+\frac{T^{4}}{\lambda^{3}}\right)\|\bar{v}\|^{2}\right) .
\end{aligned}
$$

This, together with (C.19, 39-40) and (3.24), yields

$$
\begin{aligned}
\int_{\Omega} \mid \varphi_{\alpha, \lambda, y} & +\left.\bar{v}\right|^{p-1}\left(\varphi_{\alpha, \lambda, y}+\bar{v}\right) v_{4}^{\prime}=p \alpha_{1}^{p-1} \int_{\Omega} U_{1}^{p-1} v_{4}^{\prime 2} \\
& +0\left[\|\bar{v}\|\left(\frac{T^{\frac{n-4}{2}}}{\lambda^{\frac{n-2}{2}}}+\left(\frac{(\log \lambda)^{4 / 3}}{\lambda^{2}} \text { if } n=6 ; \frac{1}{\lambda^{\frac{n+2}{n-2}}} \text { if } n \geq 7\right)\right)\right]
\end{aligned}
$$


and, from (C.33-35,37,40), (3.24) and Lemma 3.1 we deduce

$$
\left\|v_{4}^{\prime}\right\|^{2}=0\left[\|\bar{v}\|\left(T \frac{(\log \lambda)^{2 / 3}}{\lambda^{3 / 2}} \text { if } n=6 ; \frac{T}{\lambda^{3 / 2}}+\frac{1}{\lambda^{\frac{n+2}{n-2}}} \text { if } n \geq 7\right)\right] .
$$

Then, $($ C.1,19,28,31) and (D.26,31) allow to conclude

$$
\left\|v_{4}\right\|^{2}=0\left[\|\bar{v}\|\left(T \frac{(\log \lambda)^{2 / 3}}{\lambda^{3 / 2}} \text { if } n=6 ; \frac{T}{\lambda^{3 / 2}}+\frac{1}{\lambda^{\frac{n+2}{n-2}}} \text { if } n \geq 7\right)\right] .
$$

\section{Estimate of $v_{2}$}

We are now able to evaluate $\left\|v_{2}\right\|$. We proceed as previously, writing

$$
v_{2}=a^{\prime} U_{1}+b^{\prime} \frac{\partial U_{1}}{\partial \lambda_{1}}+\sum_{j=1}^{n} c_{j}^{\prime} \frac{\partial U_{1}}{\partial y_{j}^{1}}+v_{2}^{\prime}
$$

with

$$
\int_{\Omega} \nabla U_{1} \cdot \nabla v_{2}^{\prime}=\int_{\Omega} \nabla \frac{\partial U_{1}}{\partial \lambda_{1}} \cdot \nabla v_{2}^{\prime}=\int_{\Omega} \nabla \frac{\partial U_{1}}{\partial y_{j}^{1}} \cdot \nabla v_{2}^{\prime}=0 \quad 1 \leq j \leq n
$$

We multiply the gradient of (C.43) by $\nabla U_{1}, \nabla \frac{\partial U_{1}}{\partial \lambda_{1}}, \nabla \frac{\partial U_{1}}{\partial y_{j}^{1}}, 1 \leq j \leq n$, respectively, and integrate over $\Omega$, in order to obtain a linear system in the coefficients $a^{\prime}, b^{\prime}, c_{j}^{\prime}$, whose left hand terms are $\int_{\Omega} \nabla U_{1} \cdot \nabla v_{2}, \int_{\Omega} \nabla \frac{\partial U_{1}}{\partial \lambda_{1}} \cdot \nabla v_{2}, \int_{\Omega} \nabla \frac{\partial U_{1}}{\partial y_{j}^{1}} \cdot \nabla v_{2}$ respectively. Let us estimate these quantities. Using (C.14,21-23,25), we have

$$
\begin{aligned}
& \int_{\Omega} \nabla U_{1} \cdot \nabla v_{2} d x \\
& =\sum_{i=1}^{n} \int_{\Omega}-\frac{(n-2) \lambda_{1}^{\frac{n+2}{2}} x_{i}}{\left(1+\lambda_{i}^{2}|x|^{2}\right)^{n / 2}} \frac{\partial v_{2}}{\partial x_{i}} d x \\
& =-\frac{(n-2)}{2} \lambda_{1}^{\frac{n+2}{2}}\left(\sum _ { i = 1 } ^ { n - 1 } \int _ { B ^ { + } } \left[\frac{z_{i}}{\left(1+\lambda_{1}^{2}|z|^{2}\right)^{n / 2}}-\frac{n \lambda_{1}^{2}\left(\sum_{j=1}^{n-1} f_{j} z_{j}^{2} z_{n}\right) z_{i}}{\left(1+\lambda_{1}^{2}|z|^{2}\right)^{\frac{n+2}{2}}}\right.\right. \\
& \left.+0\left(\frac{\lambda_{1}^{2}|z|^{5}}{\left(1+\lambda^{2}|z|^{2}\right)^{\frac{n+2}{2}}}\right)\right] \cdot\left[T \frac{z_{i}}{|z|} \xi^{\prime}(T|z|)(\tilde{\bar{v}}(z)-\tilde{\bar{v}}(\bar{z}))+\tilde{\zeta}_{T}(z)\left(\frac{\partial \tilde{\bar{v}}}{\partial z_{i}}-\frac{\partial \tilde{\bar{v}}(\bar{z})}{\partial z_{i}}\right)\right. \\
& \left.-\left(2 f_{i} z_{i}+0\left(|z|^{2}\right)\right)\left(T \frac{z_{n}}{|z|} \xi^{\prime}(T|z|)(\tilde{\bar{v}}(z)-\tilde{\bar{v}}(\bar{z}))+\tilde{\zeta}_{T}(z)\left(\frac{\partial \tilde{\bar{v}}}{\partial z_{n}}-\frac{\partial \tilde{\bar{v}}(\bar{z})}{\partial z_{n}}\right)\right)\right] d z \\
& +\int_{B^{+}}\left[\frac{z_{n}}{\left(1+\lambda_{1}^{2}|z|^{2}\right)^{n / 2}}-\frac{n \lambda_{1}^{2}\left(\sum_{j=1}^{n-1} f_{j} z_{j}^{2}\right) z_{n}^{2}}{\left(1+\lambda_{1}^{2}|z|^{2}\right)^{\frac{n+2}{2}}}+0\left(\frac{\lambda_{1}^{2}|z|^{5}}{\left(1+\lambda_{1}^{2}|z|^{2}\right)^{\frac{n+2}{2}}}\right)\right] \\
& \left.\cdot\left[T \frac{z_{n}}{|z|} \xi^{\prime}(T|z|)(\tilde{\bar{v}}(z)-\tilde{\bar{v}}(\bar{z}))+\tilde{\zeta}_{T}(z)\left(\frac{\partial \tilde{\bar{v}}}{\partial z_{n}}-\frac{\partial \tilde{\bar{v}}(\bar{z})}{\partial z_{n}}\right)\right] d z\right) \text {. }
\end{aligned}
$$


Performing the change of variable $\bar{z} \mapsto z$ for the terms in which $\tilde{\widetilde{v}}(\bar{z})$ or $\frac{\partial \tilde{\tilde{v}}(\bar{z})}{\partial z_{i}}$ occur, and using the symmetry of $B^{+}$with respect to $z_{1}=0$, we get

$$
\int_{\Omega} \nabla U_{1} \cdot \nabla v_{2} d x=0\left(\lambda_{1}^{\frac{n+2}{2}} \int_{B^{+}} \frac{|z|^{3}}{\left(1+\lambda_{1}^{2}|z|^{2}\right)^{n / 2}}(T|\tilde{\bar{v}}(z)|+|\nabla \tilde{\bar{v}}(z)|) d z\right) .
$$

Noticing that $\int_{B^{+}} \tilde{\widetilde{v}}^{2}(z) d z=\int_{W} \bar{v}^{2} d x$ and $\int_{B^{+}}|\nabla \tilde{\tilde{v}}(z)|^{2} d z=0\left(\int_{W}|\nabla \bar{v}(x)|^{2} d x\right)$, this yields

$$
\int_{\Omega} \nabla U_{1} \cdot \nabla v_{2} d x=0\left[\lambda_{1}^{\frac{n+2}{2}}\left(\int_{B^{+}} \frac{|z|^{6}}{\left(1+\lambda_{1}^{2}|z|^{2}\right)^{n}}\right)^{1 / 2}\left(1+\frac{T}{\mu^{1 / 2}}\right)\|\bar{v}\|\right] .
$$

Using (C.6,19) and (D.58) we get finally

$$
\int_{\Omega} \nabla U_{1} \cdot \nabla v_{2} d x=0\left[\left(\frac{\left(\log \lambda_{1}\right)^{1 / 2}}{\lambda_{1}^{2}} \text { if } n=6 ; \frac{1}{\lambda_{1}^{2}} \text { if } n>6\right)\|\bar{v}\|\right] .
$$

In the same way, we have

$$
\int_{\Omega} \nabla \frac{\partial U_{1}}{\partial \lambda_{1}} \cdot \nabla v_{2} d x=0\left[\left(\frac{(\log \lambda)^{1 / 2}}{\lambda_{1}^{3}} \text { if } n=6 ; \frac{1}{\lambda_{1}^{3}} \text { if } n>6\right)\|\bar{v}\|\right] .
$$

Similarly, we compute, for $2 \leq i \leq n-1$

$$
\begin{aligned}
& \int_{\Omega} \nabla \frac{\partial U_{1}}{\partial y_{i}^{1}} \cdot \nabla v_{2} d x \\
& =\sum_{j=1}^{n} \int_{\Omega} \nabla \frac{\partial^{2} U_{1}}{\partial y_{i}^{1} \partial x_{j}} \frac{\partial v_{2}}{\partial x_{j}} d x \\
& =(n-2) \lambda_{1}^{\frac{n+2}{2}} \sum_{j=1}^{n} \int_{\Omega}\left(\frac{\delta_{i j}}{\left(1+\lambda_{1}^{2}|x|^{2}\right)^{\frac{n}{2}}}-\frac{n \lambda_{1}^{2} x_{i} x_{j}}{\left(1+\lambda_{1}^{2}|x|^{\frac{n+2}{2}}\right)}\right) \frac{\partial v_{1}}{\partial x_{j}} d x \\
& =(n-2) \lambda_{1}^{\frac{n+2}{2}}\left(\sum _ { j = 1 } ^ { n } \int _ { B ^ { + } } \left[\frac{\delta_{i j}}{\left(1+\lambda_{1}^{2}|z|^{2}\right)^{\frac{n}{2}}}-\frac{n \lambda_{1}^{2}\left(\sum_{\ell=1}^{n-1} f_{\ell} z_{\ell}^{2}\right) z_{n} \delta_{i j}}{\left(1+\lambda_{1}^{2}|z|^{2}\right)^{\frac{n+2}{2}}}\right.\right. \\
& \left.-\frac{n \lambda_{1}^{2} z_{i} z_{j}}{\left(1+\lambda_{1}^{2}|z|^{2}\right)^{\frac{n+2}{2}}}+\frac{n(n+2) \lambda_{1}^{4}\left(\sum_{\ell=1}^{n-1} f_{\ell} z_{\ell}^{2}\right) z_{i} z_{j} z_{n}}{\left(1+\lambda_{1}^{2}|z|^{2}\right)^{\frac{n+4}{2}}}+0\left(\frac{\lambda_{1}^{2}|z|^{4}}{\left(1+\lambda_{1}^{2}|z|^{2}\right)^{\frac{n+2}{2}}}\right)\right] \\
& \cdot\left[T \frac{z_{j}}{|z|} \xi^{\prime}(T|z|)(\tilde{\bar{v}}(z)-\tilde{\bar{v}}(\bar{z}))+\tilde{\zeta}_{T}(z)\left(\frac{\partial \widetilde{\bar{v}}}{\partial z_{j}}-\frac{\partial \tilde{\bar{v}}(\bar{z})}{\partial z_{j}}\right)\right. \\
& \left.-\left(2 f_{j} z_{j}+0\left(|z|^{2}\right)\right)\left(T \frac{z_{n}}{|z|} \xi^{\prime}(T|z|)(\tilde{\bar{v}}(z)-\tilde{\bar{v}}(\bar{z}))+\tilde{\xi}_{T}(z)\left(\frac{\partial \tilde{\bar{v}}}{\partial z_{n}}-\frac{\partial \tilde{\bar{v}}(\bar{z})}{\partial z_{n}}\right)\right)\right] d z \\
& +\int_{B^{+}}\left[\frac{-n \lambda_{1}^{2} z_{i} z_{n}}{\left(1+\lambda_{1}^{2}|z|^{2}\right)^{\frac{n+2}{2}}}+\frac{n(n+2) \lambda_{1}^{4}\left(\sum_{\ell=1}^{n-1} f_{\ell} z_{\ell}^{2}\right) z_{i} z_{n}^{2}}{\left(1+\lambda_{1}^{2}|z|^{2}\right)^{\frac{n+4}{2}}}+0\left(\frac{\lambda_{1}^{4}|z|^{6}}{\left(1+\lambda_{1}^{2}|z|^{2}\right)^{\frac{n+4}{2}}}\right)\right] \\
& \left.\left.\cdot\left[T \frac{z_{n}}{|z|} \xi^{\prime}(T|z|)(\tilde{\bar{v}}(z)-\tilde{\bar{v}}(\bar{z}))+\tilde{\zeta}_{T}(z)\left(\frac{\partial \widetilde{\bar{v}}}{\partial z_{n}}-\frac{\partial \tilde{\bar{v}}(\bar{z})}{\partial z_{n}}\right)\right] d z\right\}\right) .
\end{aligned}
$$


The dominating terms have no contribution to the integral since they are odd with respect to $z_{1}$, and we get

$$
\begin{aligned}
\int_{\Omega} \nabla \frac{\partial U_{1}}{\partial y_{i}^{1}} \cdot \nabla v_{2} d x & =0\left(\lambda_{1}^{\frac{n+2}{2}} \int_{B^{+}} \frac{|z|^{2}}{\left(1+\lambda_{1}^{2}|z|^{2}\right)^{n / 2}}(T|\tilde{\tilde{v}}(z)|+|\nabla \tilde{\bar{v}}(z)|) d z\right) \\
& =\left(\lambda_{1}^{\frac{n+2}{2}}\left(\int_{B^{+}} \frac{|z|^{4}}{\left(1+\lambda_{1}^{2}|z|^{2}\right)^{n}}\right)^{1 / 2}\left(1+\frac{T}{\mu^{1 / 2}}\right)\|\bar{v}\|\right) .
\end{aligned}
$$

Thus, using (C.6,19) and (D.59), we obtain

$$
\int_{\Omega} \nabla \frac{\partial U_{1}}{\partial y_{i}^{1}} \cdot \nabla v_{2} d x=0\left(\frac{\|\bar{v}\|}{\lambda_{1}}\right) \quad 2 \leq i \leq n-1
$$

It remains to estimate $\int_{\Omega} \nabla \frac{\partial U_{1}}{\partial y_{1}^{1}} . \nabla v_{2} d x$. We remark that, since $\bar{v} \in E_{\lambda, y}$

$$
\int_{\Omega} \nabla \frac{\partial U_{1}}{\partial y_{1}^{1}} \cdot \nabla v_{2}=-\int_{\Omega} \nabla \frac{\partial U_{1}}{\partial y_{1}^{1}} \cdot \nabla v_{1}-\int_{\Omega} \nabla \frac{\partial U_{1}}{\partial y_{1}^{1}} \cdot \nabla v_{3}
$$

On one hand

$$
\int_{\Omega} \nabla \frac{\partial U_{1}}{\partial y_{1}^{1}} \cdot \nabla v_{3}=0\left[\|\bar{v}\|\left(\int_{|x| \geq \frac{1}{4}}\left|\nabla \frac{\partial U_{1}}{\partial y_{1}^{1}}\right|^{2}\right)^{1 / 2}\right] .
$$

Indeed, according to (C.21)

$$
\int_{\Omega}\left|\nabla v_{3}\right|^{2}=0\left(\int_{\Omega}|\nabla \bar{v}|^{2}+T^{2} \int_{\Omega} \bar{v}^{2}\right)=0\left(\|\bar{v}\|^{2}\right)
$$

because of (C.6,19). (D.29) then yields

$$
\int_{\Omega} \nabla \frac{\partial U_{1}}{\partial y_{1}^{1}} \cdot \nabla v_{3}=0\left(\frac{T^{\frac{n}{2}}}{\lambda_{1}^{\frac{n-2}{2}}}\|\bar{v}\|\right) .
$$


On the other hand, using (C.14,21-23,25)

$$
\begin{aligned}
& \int_{\Omega} \nabla \frac{\partial U_{1}}{\partial y_{1}^{1}} \cdot \nabla v_{1} \\
& =\sum_{j=1}^{n} \int_{\Omega} \frac{\partial^{2} U_{1}}{\partial y_{1}^{1} \partial x_{j}} \frac{\partial v_{1}}{\partial x_{j}} d x \\
& =(n-2) \lambda_{1}^{\frac{n+2}{2}} \sum_{j=1}^{n} \int_{\Omega}\left(\frac{\delta_{i j}}{\left(1+\lambda_{1}^{2}|x|^{2}\right)^{\frac{n}{2}}}-\frac{n \lambda_{1}^{2} x_{1} x_{j}}{\left(1+\lambda_{2}^{2}|x|^{2}\right)^{n+2}}\right) \frac{\partial v_{1}}{\partial x_{i}} d x \\
& =(n-2) \lambda_{1}^{\frac{n+2}{2}}\left(\sum _ { j = 1 } ^ { n - 1 } \int _ { B ^ { + } } \left[\frac{\delta_{1 j}}{\left(1+\lambda_{1}^{2}|z|^{2}\right)^{\frac{n}{2}}}-\frac{n \lambda_{1}^{2}\left(\sum_{\ell=1}^{n-1} f_{\ell} z_{\ell}^{2}\right) z_{n}}{\left(1+\lambda_{1}^{2}|z|^{2}\right)^{\frac{n+2}{2}}} \delta_{1 j}\right.\right. \\
& \left.-\frac{n \lambda_{1}^{2} z_{1} z_{j}}{\left(1+\lambda_{1}^{2}|z|^{2}\right)^{\frac{n+2}{2}}}+\frac{n(n+2) \lambda_{1}^{4}\left(\sum_{\ell=1}^{n-1} f_{\ell} z_{\ell}^{2}\right) z_{1} z_{j} z_{n}}{\left(1+\lambda_{1}^{2}|z|^{2}\right)^{\frac{n+4}{2}}}+0\left(\frac{\lambda_{1}^{2}|z|^{4}}{\left(1+\lambda_{1}^{2}|z|^{2}\right)^{\frac{n+2}{2}}}\right)\right] \\
& \cdot\left[T \frac{z_{j}}{|z|} \xi^{\prime}(T|z|)(\widetilde{\bar{v}}(z)+\widetilde{\widetilde{v}}(\bar{z}))+\tilde{\zeta}_{T}(z)\left(\frac{\partial \widetilde{\widetilde{v}}}{\partial z_{j}}+\frac{\partial \widetilde{\widetilde{v}}(\bar{z})}{\partial z_{j}}\right)\right. \\
& \left.-\left(2 f_{j} z_{j}+0\left(|z|^{2}\right)\right)\left(T \frac{z_{n}}{|z|} \xi^{\prime}(T|z|)(\widetilde{\bar{v}}(z)+\widetilde{\bar{v}}(\bar{z}))+\tilde{\zeta}_{T}(z)\left(\frac{\partial \widetilde{\tilde{v}}}{\partial z_{n}}+\frac{\partial \widetilde{\widetilde{v}}(\bar{z})}{\partial z_{n}}\right)\right)\right] d z \\
& +\int_{B^{+}}\left[-\frac{n \lambda_{1}^{2} z_{1} z_{n}}{\left(1+\lambda_{1}^{2}|z|^{2}\right)^{\frac{n+2}{2}}}+n(n+2) \lambda_{1}^{4} \frac{\left(\sum_{\ell=1}^{n-1} f_{\ell} z_{\ell}^{2}\right) z_{1} z_{n}^{2}}{\left(1+\lambda_{1}^{2}|z|^{2}\right)^{\frac{n+4}{2}}}+0\left(\frac{\lambda_{1}^{4}|z|^{6}}{\left(1+\lambda_{1}^{2}|z|^{2}\right)^{\frac{n+4}{2}}}\right)\right] \\
& \left.\left[T \frac{z_{n}}{|z|} \xi^{\prime}(T|z|)(\widetilde{\bar{v}}(z)+\widetilde{\widetilde{v}}(\bar{z}))+\tilde{\zeta}_{T}(z)\left(\frac{\partial \widetilde{\widetilde{v}}}{\partial z_{n}}+\frac{\partial \widetilde{\widetilde{v}}(\bar{z})}{\partial z_{n}}\right)\right] d z\right) \\
& =0\left(\lambda_{1}^{\frac{n+2}{2}} \int_{B^{+}} \frac{|z|^{2}}{\left(1+\lambda_{1}^{2}|z|^{2}\right)^{\frac{n}{2}}}(T|\widetilde{\bar{v}}(z)|+|\nabla \widetilde{\widetilde{v}}(z)|) d z\right) \text {. }
\end{aligned}
$$

As previously, for the estimate of $\int_{\Omega} \nabla \frac{\partial U_{1}}{\partial y_{1}^{1}} . \nabla v_{2} d x$, we obtain, using Schwarz inequality, (C.6,19) and (D.59)

$$
\int_{\Omega} \nabla \frac{\partial U_{1}}{\partial y_{1}^{1}} \cdot \nabla v_{1}=0\left(\frac{\|\bar{v}\|}{\lambda_{1}}\right)
$$

Combining (C.47-49) and (C.19), we get

$$
\int_{\Omega} \nabla \frac{\partial U_{1}}{\partial y_{1}^{1}} \cdot \nabla v_{2}=0\left(\frac{\|\bar{v}\|}{\lambda_{1}}\right)
$$


From (C.43-44,49) and (D.27) we deduce

$$
\left\{\begin{array}{l}
a^{\prime}=0\left[\left(\frac{(\log \lambda)^{1 / 2}}{\lambda^{2}} \text { if } n=6 ; \frac{1}{\lambda^{2}} \text { if } n>6\right)\|\bar{v}\|\right] \\
b^{\prime}=0\left[\left(\frac{(\log \lambda)^{1 / 2}}{\lambda} \text { if } n=6 ; \frac{1}{\lambda} \text { if } n>6\right)\|\bar{v}\|\right] \\
c_{j}^{\prime}=0\left[\frac{\|\bar{v}\|}{\lambda^{3}}\right] .
\end{array}\right.
$$

It only remains to estimate $v_{2}^{\prime}$, taking $w=v_{2}^{\prime}$ in (3.20). On the right hand side, we find

$$
\begin{aligned}
\mathcal{R}^{\prime} & =\sum_{i=1}^{k}\left(A_{i} \int_{\Omega} \nabla U_{i} \cdot \nabla v_{2}^{\prime}+B_{i} \int_{\Omega} \nabla \frac{\partial U_{i}}{\partial \lambda_{i}} \cdot \nabla v_{2}^{\prime}+\sum_{j=1}^{n-1} C_{i, j} \int_{\Omega} \nabla \frac{\partial U_{i}}{\partial \tau_{j}^{i}} \cdot \nabla v_{2}^{\prime}\right) \\
& =\sum_{i=2}^{k}\left(\int_{\Omega}\left(A_{i} \nabla U_{i}+B_{i} \nabla \frac{\partial U_{i}}{\partial \lambda_{i}}+\sum_{j=1}^{n-1} C_{i, j} \nabla \frac{\partial U_{i}}{\partial \tau_{j}^{i}}\right) \cdot \nabla\left(v_{2}-a^{\prime} U_{1}-b^{\prime} \frac{\partial U_{1}}{\partial \lambda_{1}}-\sum_{j=1}^{\ell} c_{\ell}^{\prime} \frac{\partial U_{1}}{\partial y_{\ell}^{1}}\right)\right)
\end{aligned}
$$

because of (C.42-43). It is easy to see, from the definition of $v_{2}$ in (C.21), that, for $2 \leq i \leq n$

$$
\begin{aligned}
\int_{\Omega} \nabla U_{i} \cdot \nabla v_{2} & =0\left(\frac{1}{\lambda_{i}^{\frac{n-2}{2}}} \int_{\Omega}\left|\nabla v_{2}\right|\right) \\
& =0\left(\frac{1}{\lambda_{i}^{\frac{n-2}{2}}} \int_{|x| \leq \frac{2}{T}}(|\nabla \bar{v}|+T|\bar{v}|)\right) \\
& =0\left[\frac{1}{\lambda_{i}^{\frac{n-2}{2}}} \frac{1}{T^{\frac{n}{2}}}\left(\left(\int_{\Omega}|\nabla \bar{v}|^{2}\right)^{1 / 2}+T\left(\int_{\Omega} \bar{v}^{2}\right)^{1 / 2}\right)\right]
\end{aligned}
$$

using Schwarz inequality. Finally, taking account of (C.6,19), we find

$$
\left\{\begin{array}{l}
\int_{\Omega} \nabla U_{i} \cdot \nabla v_{2}=0\left(\frac{1}{\lambda^{\frac{n-2}{2}} T^{\frac{n}{2}}}\|\bar{v}\|\right) \\
\int_{\Omega} \frac{\partial U_{i}}{\partial \lambda_{i}} \cdot \nabla v_{2}=0\left(\frac{1}{\lambda^{\frac{n}{2}} T^{\frac{n}{2}}}\|\bar{v}\|\right) \\
\int_{\Omega} \frac{\partial U_{i}}{\partial \tau_{j}^{i}} \cdot \nabla v_{2}=0\left(\frac{1}{\lambda^{\frac{n-2}{2}} T^{\frac{n}{2}}}\|\bar{v}\|\right)
\end{array}\right.
$$

This together with $(\mathrm{C} .8,19,52)$ and (D.26) leads to

$$
\mathcal{R}^{\prime}=0\left[\left(|\eta|+\frac{1}{\lambda}\right) \frac{1}{\lambda^{\frac{n-2}{2}} T^{\frac{n}{2}}}\|\bar{v}\|\right] .
$$


The left hand side is equal to

(C.55)

$$
\mathcal{L}=\int_{\Omega} \nabla\left(\varphi_{\alpha, \lambda, y}+\bar{v}\right) \cdot \nabla v_{2}^{\prime}+\mu \int_{\Omega}\left(\varphi_{\alpha, \lambda, y}+\bar{v}\right) v_{2}^{\prime}-\int_{\Omega}\left|\varphi_{\alpha, \lambda, y}+\bar{v}\right|^{p-1}\left(\varphi_{\alpha, \lambda, y}+\bar{v}\right) v_{2}^{\prime} .
$$

We begin by computing the first integral.

$$
\begin{aligned}
& \int_{\Omega} \nabla\left(\varphi_{\alpha, \lambda, y}+\bar{v}\right) \cdot \nabla v_{2}^{\prime} \\
& =\sum_{i=1}^{k} \alpha_{i} \int_{\Omega} \nabla U_{i} \cdot \nabla v_{2}^{\prime}+\int_{\Omega} \nabla \bar{v} \cdot \nabla v_{2}^{\prime} \\
& =\sum_{i=2}^{k} \alpha_{i} \int_{\Omega} \nabla U_{i} \cdot \nabla\left(v_{2}-a^{\prime} U_{1}-b^{\prime} \frac{\partial U_{1}}{\partial \lambda_{1}}-\sum_{j=1}^{n} c_{j}^{\prime} \frac{\partial U_{1}}{\partial y_{j}^{1}}\right)+\int_{\Omega} \nabla\left(v_{1}+v_{2}^{\prime}+v_{3}\right) \cdot \nabla v_{2}^{\prime} \\
& =\sum_{i=2}^{k} \alpha_{i}\left(\int_{\Omega} \nabla U_{i} \cdot \nabla v_{2}-a \int_{\Omega} \nabla U_{i} \cdot \nabla U_{1}-b^{\prime} \int_{\Omega} \nabla U_{i} \cdot \nabla \frac{\partial U_{1}}{\partial \lambda_{1}}-\sum_{j=1}^{n} c_{j}^{\prime} \int_{\Omega} \nabla U_{i} \cdot \nabla \frac{\partial U_{1}}{\partial y_{j}^{1}}\right) \\
& +\int_{\Omega}\left|\nabla v_{2}^{\prime}\right|^{2}+\int_{\Omega} \nabla\left(v_{1}+v_{3}\right) \cdot \nabla v_{2}-\int_{\Omega} \nabla\left(\bar{v}-v_{2}\right) \cdot \nabla\left(a^{\prime} U_{1}+b^{\prime} \frac{\partial U_{1}}{\partial \lambda_{1}}+\sum_{j=1}^{n} c_{j}^{\prime} \frac{\partial U_{1}}{\partial y_{j}^{1}}\right) \\
& =\int_{\Omega}\left|\nabla v_{2}^{\prime}\right|^{2}+\int_{\Omega} \nabla\left(v_{1}+v_{3}\right) \cdot \nabla v_{2}+0\left(\|\bar{v}\|\left(\frac{1}{\lambda^{\frac{n-2}{2}} T^{\frac{n}{2}}}+\frac{1}{\lambda^{5}}\right)\right)
\end{aligned}
$$

using (C.19,43-47,51-53) and (D.26). Moreover

$$
\begin{aligned}
& \int_{\Omega} \nabla v_{1} \cdot \nabla v_{2} \\
& =\sum_{i=1}^{n} \int_{\Omega} \frac{\partial v_{1}}{\partial x_{i}} \frac{\partial v_{2}}{\partial x_{i}} d x \\
& =\sum_{i=1}^{n-1} \int_{B^{+}}\left[T \frac{z_{i}}{|z|} \xi^{\prime}(T|z|)(\widetilde{\bar{v}}(z)+\widetilde{\bar{v}}(\bar{z}))+\tilde{\zeta}_{T}(z)\left(\frac{\partial \widetilde{\bar{v}}}{\partial z_{i}}+\frac{\partial \widetilde{\widetilde{v}}(\bar{z})}{\partial z_{i}}\right)\right. \\
& \left.-\left(2 f_{i} z_{i}+0\left(|z|^{2}\right)\right)\left(T \frac{z_{n}}{|z|} \xi^{\prime}(T|z|)\right)(\widetilde{\bar{v}}(z)+\widetilde{\bar{v}}(\bar{z}))+\tilde{\zeta}_{T}(z)\left(\frac{\partial \widetilde{\widetilde{v}}}{\partial z_{n}}+\frac{\partial \widetilde{\widetilde{v}}(\bar{z})}{\partial z_{n}}\right)\right] \\
& \cdot\left[T \frac{z_{i}}{|z|} \xi^{\prime}(T|z|)(\tilde{\bar{v}}(z)-\widetilde{\widetilde{v}}(\bar{z}))+\tilde{\zeta}_{T}(z)\left(\frac{\partial \widetilde{\tilde{v}}}{\partial z_{i}}-\frac{\partial \widetilde{\tilde{v}}(\bar{z})}{\partial z_{i}}\right)\right. \\
& \left.-\left(2 f_{i} z_{i}+0\left(|z|^{2}\right)\right)\left(T \frac{z_{n}}{|z|} \xi^{\prime}(T|z|)\right)(\widetilde{\bar{v}}(z)-\widetilde{\tilde{v}}(\bar{z}))+\tilde{\zeta}_{T}(z)\left(\frac{\partial \widetilde{\widetilde{v}}}{\partial z_{n}}-\frac{\partial \widetilde{\widetilde{v}}(\bar{z})}{\partial z_{n}}\right)\right] d z
\end{aligned}
$$




$$
\begin{aligned}
& +\int_{B^{+}}\left[T \frac{z_{n}}{|z|} \xi^{\prime}(T|z|)(\widetilde{\bar{v}}(z)+\widetilde{\bar{v}}(\bar{z}))+\tilde{\zeta}_{T}(z)\left(\frac{\partial \widetilde{\widetilde{v}}}{\partial z_{n}}+\frac{\partial \widetilde{\widetilde{v}}(\bar{z})}{\partial z_{n}}\right)\right] \\
& \cdot\left[T \frac{z_{n}}{|z|} \xi^{\prime}(T|z|)(\widetilde{\bar{v}}(z)-\widetilde{\widetilde{v}}(\bar{z}))+\tilde{\zeta}_{T}(z)\left(\frac{\partial \widetilde{\bar{v}}}{\partial z_{n}}-\frac{\partial \widetilde{\tilde{v}}(\bar{z})}{\partial z_{n}}\right)\right] d z \\
& =0\left(\int_{|z| \leq \frac{1}{T}}|z|^{2}\left(|\nabla \widetilde{\tilde{v}}|^{2}+T^{2}|\widetilde{\bar{v}}|^{2}\right)\right) \text { using oddness properties with respect to } z_{1} \\
& =0\left(\frac{1}{T^{2}}\left(\int_{\Omega}|\nabla \bar{v}|^{2}+T^{2} \bar{v}^{2}\right)\right) \text { coming back to the variable } x \text { through (C.14) } \\
& =0\left(\frac{1}{T^{2}}\|\bar{v}\|^{2}\right)
\end{aligned}
$$

because of $(\mathrm{C} .1,19)$. We have also, for any $\sigma>0$

$$
\left|\int_{\Omega} \nabla v_{3} \cdot \nabla v_{2}\right|=\left|\int_{\Omega} \nabla v_{4} \cdot \nabla v_{2}\right| \leq\left(\int_{\Omega}\left|\nabla v_{4}\right|^{2}\right)^{1 / 2}\left(\int_{\Omega}\left|\nabla v_{2}\right|^{2}\right)^{1 / 2} \leq \sigma \int_{\Omega}\left|\nabla v_{2}\right|^{2}+\frac{1}{4 \sigma}\left\|v_{4}\right\|^{2} .
$$

Thus, using (C.19,42) and (3.24), we get

$$
\text { (C.56) }
$$

$$
\begin{aligned}
& \int_{\Omega} \nabla\left(\varphi_{\alpha, \lambda, y}+\bar{v}\right) \cdot \nabla v_{2}^{\prime}=(1+0(\sigma)) \int_{\Omega}\left|\nabla v_{2}^{\prime}\right|^{2} \\
& +0\left[\|\bar{v}\|\left(\left(\frac{(\log \lambda)^{\frac{2}{3}}}{\lambda T^{2}}+\frac{T(\log \lambda)^{\frac{2}{3}}}{\sigma \lambda^{3 / 2}}\right) \text { if } n=6 ;\left(\frac{1}{\lambda T^{2}}+\frac{1}{\sigma}\left(\frac{T}{\lambda^{3 / 2}}+\frac{1}{\lambda^{\frac{n+2}{n-2}}}\right)\right) \text { if } n \geq 7\right)\right] .
\end{aligned}
$$

We compute now the second integral in (C.55). According to (C.43), we have (C.57)

$$
\begin{aligned}
& \mu \int_{\Omega}\left(\varphi_{\alpha, \lambda, y}+\bar{v}\right) v_{2}^{\prime} \\
& =\mu \sum_{i=1}^{n} \alpha_{i} \int_{\Omega} U_{i}\left(v_{2}-a^{\prime} U_{1}-b^{\prime} \frac{\partial U_{1}}{\partial \lambda_{1}}-\sum_{j=1}^{n} c_{j}^{\prime} \frac{\partial U_{1}}{\partial y_{j}^{1}}\right)+\mu \int_{\Omega} v_{2}^{\prime 2} \\
& +\mu \int_{\Omega}\left(v_{1}+v_{3}+a^{\prime} U_{1}+b^{\prime} \frac{\partial U_{1}}{\partial \lambda_{1}}+\sum_{j=1}^{n} c_{j}^{\prime} \frac{\partial U_{1}}{\partial y_{j}^{1}}\right)\left(v_{2}-a^{\prime} U_{1}-b^{\prime} \frac{\partial U_{1}}{\partial \lambda_{1}}-\sum_{j=1}^{n} c_{j}^{\prime} \frac{\partial U_{1}}{\partial y_{j}^{1}}\right) .
\end{aligned}
$$


Using (C.21-22,25) and oddness properties with respect to $z_{1}$, we may write

$$
\begin{aligned}
& \int_{\Omega} U_{1} v_{2}=\int_{B^{+}} \lambda_{1}^{\frac{n-2}{2}}\left[\frac{1}{\left(1+\lambda_{1}^{2}|z|^{2}\right)^{\frac{n-2}{2}}}-\frac{(n-2) \lambda_{1}^{2}\left(\sum_{i=1}^{n-1} f_{i} z_{i}^{2}\right) z_{n}}{\left(1+\lambda_{1}^{2}|z|^{2}\right)^{n / 2}}+0\left(\frac{\lambda_{1}^{2}|z|^{4}}{\left(1+\lambda_{1}^{2}|z|^{2}\right)^{\frac{n}{2}}}\right)\right] \\
& \cdot \frac{1}{2} \tilde{\zeta}_{T}(z)(\widetilde{\bar{v}}(z)-\widetilde{\bar{v}}(\bar{z})) d z \\
& =0\left(\lambda_{1}^{\frac{n+2}{2}} \int_{|z| \leq \frac{1}{T}} \frac{|z|^{4}}{\left(1+\lambda_{1}^{2}|z|^{2}\right)^{\frac{n}{2}}}|\tilde{\bar{v}}(z)| d z\right) \\
& =0\left[\lambda_{1}^{\frac{n+2}{2}}\left(\int_{|z| \leq \frac{1}{T}} \frac{|z|^{8} d z}{\left(1+\lambda_{1}^{2}|z|^{2}\right)^{n}}\right)^{1 / 2}\left(\int_{B^{+}} \widetilde{\bar{v}}^{2}(z) d z\right)^{1 / 2}\right] \\
& =0\left[\frac{\lambda_{1}^{\frac{n+2}{2}}}{\mu^{1 / 2}}\|\bar{v}\|\left(\frac{T^{\frac{n-8}{2}}}{\lambda_{1}^{n}} \text { if } n \leq 7 ; \frac{\left(\log \lambda_{1}\right)^{1 / 2}}{\lambda_{1}^{8}} \text { if } n=8 ; \frac{1}{\lambda_{1}^{\frac{n+8}{2}}} \text { if } n \geq 9\right)\right]
\end{aligned}
$$

using (D.60). Therefore, (C.6) yields

$$
\mu \int_{\Omega} U_{1} v_{2}=0\left[\left(\frac{T^{\frac{8-n}{2}}}{\lambda^{\frac{n-3}{2}}} \text { if } n=6,7 ; \frac{(\log \lambda)^{1 / 2}}{\lambda^{5 / 2}} \text { if } n=8 ; \frac{1}{\lambda^{5 / 2}}\right)\|\bar{v}\|\right]
$$

For $2 \leq i \leq n$, we have also

$$
\mu \int_{\Omega} U_{i} v_{2}=0\left(\frac{\mu}{\lambda^{\frac{n-2}{2}}} \int_{\Omega} \mid \bar{v} \|\right)=0\left(\frac{\mu^{1 / 2}}{\lambda^{\frac{n-2}{2}}}\|\bar{v}\|\right)
$$

so that, taking account of (C.1)

$$
\mu \int_{\Omega} U_{i} v_{2}=0\left(\frac{\|\bar{v}\|}{\lambda^{\frac{n-3}{2}}}\right) \quad 2 \leq i \leq n
$$

Because of oddness with respect to $z_{1}$ - see (C.21) - we know that

$$
\int_{\Omega} v_{1} v_{2}=\int_{B^{+}} \frac{1}{4} \tilde{\zeta}_{T}^{2}(z)\left(\tilde{\bar{v}}^{2}(z)-\widetilde{\bar{v}}^{2}(\bar{z})\right) d z=0
$$

We write

$$
\mu \int_{\Omega} v_{2} v_{3}=\mu \int_{\Omega} v_{2} v_{4}=\mu \int_{\Omega}\left(v_{2}^{\prime}+a^{\prime} U_{1}+b^{\prime} \frac{\partial U_{1}}{\partial \lambda_{1}}+\sum_{j=1}^{n} c_{j}^{\prime} \frac{\partial U_{1}}{\partial y_{j}^{\prime}}\right) v_{4}
$$

On one hand

$$
\left|\mu \int_{\Omega} v_{2}^{\prime} v_{4}\right| \leq \mu^{1 / 2}\left(\int_{\Omega} v_{2}^{\prime 2}\right)^{1 / 2} \mu^{1 / 2}\left(\int_{\Omega} v_{4}^{2}\right)^{1 / 2} \leq \sigma \mu \int_{\Omega} v_{2}^{\prime 2}+\frac{1}{4 \sigma}\left\|v_{4}\right\|^{2} .
$$


On the other hand

$$
\int_{\Omega} U_{1} v_{4}=0\left(\frac{T^{\frac{n-4}{2}}}{\lambda^{\frac{n}{2}}}\|\bar{v}\|\right) \text { as given by (C.36) }
$$

and similar computations show that

$$
\int_{\Omega} \frac{\partial U_{1}}{\partial \lambda_{1}} v_{4}=0\left(\frac{T^{\frac{n-4}{2}}}{\lambda^{\frac{n+2}{2}}}\|\bar{v}\|\right) \quad \int_{\Omega} \frac{\partial U_{1}}{\partial y_{j}^{1}} v_{4}=0\left(\frac{T^{\frac{n-2}{2}}}{\lambda^{\frac{n-2}{2}}}\|\bar{v}\|\right) .
$$

Note that the same estimates obviously hold for $v_{3}$ instead of $v_{4}$. Lastly, using (C.21-22), (C.1) and (D.31)

$$
\begin{aligned}
\mu \int_{\Omega}\left(v_{2}-v_{1}\right) & \left(a^{\prime} U_{1}+b^{\prime} \frac{\partial U_{1}}{\partial \lambda_{1}}+\sum_{j=1}^{n} c_{j}^{\prime} \frac{\partial U_{1}}{\partial y_{j}^{\prime}}\right) \\
& =-\mu \int_{B^{+}} \tilde{\zeta}_{T}(z) \tilde{\bar{v}}(\bar{z})\left(a^{\prime} \tilde{U}_{1}+b^{\prime} \frac{\widetilde{\partial U_{1}}}{\partial \lambda_{1}}+\sum_{j=1}^{n} c_{j}^{\prime} \frac{\widetilde{\partial U_{1}}}{\partial y_{j}^{\prime}}\right) d z \\
& =0\left(\lambda^{1 / 2}\|\bar{v}\|\left(\frac{\left|a^{\prime}\right|}{\lambda}+\frac{\left|b^{\prime}\right|}{\lambda^{2}}+\sum_{j=1}^{n}\left|c_{j}^{\prime}\right|\right)\right) .
\end{aligned}
$$

Collecting this last estimate with (C.58-63,52) (3.24) and (D.21-23,31), we deduce from (C.57)

$$
\begin{aligned}
\mu \int_{\Omega}\left(\varphi_{\alpha, \lambda, y}+\bar{v}\right) v_{2}^{\prime} & =\mu(1+0(\sigma)) \int_{\Omega} v_{2}^{\prime 2} \\
& +0\left[\frac{1}{\sigma}\left(\frac{T(\log \lambda)^{2 / 3}}{\lambda^{3 / 2}} \text { if } n=6 ; \frac{T}{\lambda^{3 / 2}}+\frac{1}{\lambda^{\frac{n+2}{n-2}}} \text { if } n \geq 7\right)\|\bar{v}\|\right] .
\end{aligned}
$$

It only remains to estimate the third integral in (C.55), i.e.

$$
\begin{aligned}
& \int_{\Omega}\left|\varphi_{\alpha, \lambda, y}+\bar{v}\right|^{p-1}\left(\varphi_{\alpha, \lambda, y}+\bar{v}\right) v_{2}^{\prime} \\
& =\int_{\Omega}\left(\left(\alpha_{1} U_{1}\right)^{p}+p\left(\alpha_{1} U_{1}\right)^{p-1}\left(\sum_{j=2}^{k} \alpha_{j} U_{j}+\bar{v}\right)+0\left(\sum_{j=2}^{k} U_{j=2}^{p} U_{j}^{p}+|\bar{v}|^{p}\right)\right) v_{2}^{\prime} \\
& =\int_{\Omega}\left(\alpha_{1}^{p} U_{1}^{p}+p\left(\alpha_{1} U_{1}\right)^{p-1}\left(\sum_{j=2}^{n} \alpha_{j} U_{j}\right)+0\left(\sum_{j=2}^{n} U_{j}^{p}+|\bar{v}|^{p}\right)\right) \\
& +p \alpha_{1}^{p-1} \int_{\Omega} U_{1}^{p-1} v_{2}^{\prime 2}+p \alpha_{1}^{p-1} \int_{\Omega} U_{1}^{p-1}\left(\bar{v}-v_{2}^{\prime}\right) v_{2}^{\prime} .
\end{aligned}
$$


We have, for $2 \leq j \leq k$, using Hölder's inequality

$$
\int_{\Omega} U_{1}^{p-1} U_{j} v_{2}=0\left[\frac{1}{\lambda_{j}^{\frac{n-2}{2}}}\left(\int_{\Omega}\left|v_{2}\right|^{p+1}\right)^{\frac{1}{p+1}}\left(\int_{\Omega} U_{1}^{\frac{(p-1)(p+1)}{p}}\right)^{\frac{p}{p+1}}\right]
$$

and, from (C.21)

$$
\begin{aligned}
\int_{\Omega}\left|v_{2}\right|^{p+1} & =\int_{B^{+}} \frac{1}{2^{p+1}} \tilde{\zeta}_{T}^{p+1}(z)|\widetilde{\widetilde{v}}(z)-\widetilde{\tilde{v}}(\bar{z})|^{p+1} d z \\
& =0\left(\int_{B^{+}}|\widetilde{\bar{v}}(z)|^{p+1} d z\right) \\
& =0\left(\int_{W}|\bar{v}|^{p+1}\right) .
\end{aligned}
$$

Therefore, Sobolev embedding theorem and (D.38) yield

$$
\int_{\Omega} U_{1}^{p-1} U_{j} v_{2}=0\left[\|\bar{v}\|\left(\frac{(\log \lambda)^{2 / 3}}{\lambda^{4}} \text { if } n=6 ; \frac{1}{\lambda^{\frac{n+2}{2}}} \text { if } n \geq 7\right)\right] .
$$

Because of (C.21) and (C.6), we have also, for $2 \leq j \leq k$

$$
\int_{\Omega} U_{j}^{p}\left|v_{2}\right|=0\left(\frac{1}{\lambda_{j}^{\frac{n+2}{2}}} \int_{\Omega}\left|v_{2}\right|\right)=0\left(\frac{1}{\lambda_{j}^{\frac{n+2}{2}}} \int_{\substack{x \in \Omega \\|x| \leq \frac{2}{T}}}|\bar{v}|\right)=0\left(\frac{\|\bar{v}\|}{\lambda_{j}^{\frac{n+3}{2}} T^{\frac{n}{2}}}\right)
$$

and, through a change of variables $x \rightarrow z$, it follows easily from (C.21) that

$$
\int_{\Omega}|\bar{v}|^{p} v_{2}=0\left(\|\bar{v}\|^{p+1}\right) \text {. }
$$

Thus, (C.19, 38, 52) (3.24) and (D.42-43) lead to (C.65)

$$
\begin{gathered}
\int_{\Omega}\left(p\left(\alpha_{1} U_{1}\right)^{p-1}\left(\sum_{j=2}^{k} \alpha_{j} U_{j}\right)+0\left(\sum_{j=2}^{k} U_{j}^{p}+|\bar{v}|^{p}\right)\right)\left(v_{2}-a^{\prime} U_{1}-b^{\prime} \frac{\partial U_{1}}{\partial \lambda}-\sum_{j=1}^{n} c_{j}^{\prime} \frac{\partial U_{1}}{\partial y_{j}^{1}}\right) \\
=0\left[\|\bar{v}\|\left(\frac{(\log \lambda)^{4 / 3}}{\lambda^{2}} \text { if } n=6 ; \frac{1}{\lambda^{\frac{n+2}{n-2}}} \text { if } n \geq 7\right)\right]
\end{gathered}
$$

noticing that $\frac{\partial U_{1}}{\partial \lambda_{1}}=0\left(\frac{U_{1}}{\lambda_{1}}\right)$ and $\frac{\partial U_{1}}{\partial y_{j}^{1}}=0\left(\lambda_{1} U_{1}\right)$. Then, we compute

$$
\begin{aligned}
& \int_{\Omega} U_{1}^{p}\left(a^{\prime} U_{1}+b^{\prime} \frac{\partial U_{1}}{\partial \lambda_{1}}+\sum_{j=1}^{n} c_{j}^{\prime} \frac{\partial U_{1}}{\partial y_{j}^{1}}\right) \\
& =0\left(\left|a^{\prime}\right|+\frac{\left|b^{\prime}\right|}{\lambda_{1}^{2}}+\sum_{j=1}^{n} \frac{\left|c_{j}^{\prime}\right|}{\lambda_{1}}\right) \quad \text { according to (D.17-19) } \\
& =0\left[\left(\frac{\left(\log \lambda_{1}\right)^{1 / 2}}{\lambda_{1}^{2}} \text { if } n=6 ; \frac{1}{\lambda_{1}^{2}} \text { if } n \geq 7\right)\|\bar{v}\|\right] \quad \text { according to (C.52) }
\end{aligned}
$$


and, in view of (C.21-22,25)

$$
\begin{aligned}
& \int_{\Omega} U_{1}^{p} v_{2} \\
& =\int_{B^{+}} \lambda_{1}^{\frac{n+2}{2}}\left(\frac{1}{\left(1+\lambda_{1}^{2}|z|^{2}\right)^{\frac{n+2}{2}}}-\frac{(n+2) \lambda_{1}^{2}\left(\sum_{i=1}^{n-1} f_{i}^{2}\right) z_{n}}{\left(1+\lambda_{1}^{2}|z|^{2}\right)^{\frac{n+4}{2}}}+0\left(\frac{\lambda_{1}^{2}|z|^{4}}{\left(1+\lambda_{1}^{2}|z|^{2}\right)^{\frac{n+4}{2}}}\right)\right) \\
& \quad \cdot \frac{1}{2} \tilde{\zeta}_{T}(z)(\tilde{\bar{v}}(z)-\widetilde{\bar{v}}(\bar{z})) d z \\
& =0\left(\lambda_{1}^{\frac{n+6}{2}} \int_{B^{+}} \frac{|z|^{4}}{\left(1+\lambda_{1}^{2}|z|^{2}\right)^{\frac{n+4}{2}}}|\widetilde{\tilde{v}}(z)| d z\right) \\
& =0\left[\lambda_{1}^{\frac{n+6}{2}}\left(\int_{B^{+}} \frac{|z|^{8} d z}{\left(1+\lambda_{1}^{2}|z|^{2}\right)^{n+4}}\right)^{1 / 2}\left(\int_{B^{+}} \widetilde{\bar{v}}^{2}(z) d z\right)^{1 / 2}\right]
\end{aligned}
$$

where we used oddness properties with respect to $z_{1}$. Therefore, (C.6) and (D.61) imply

$$
\int_{\Omega} U_{1}^{p} v_{2}=0\left(\frac{\|\bar{v}\|}{\lambda_{1}^{3 / 2}}\right)
$$

Thus, we obtain

$$
\int_{\Omega} \alpha_{1}^{p} U_{1}^{p}\left(v_{2}-a^{\prime} U_{1}-b^{\prime} \frac{\partial U_{1}}{\partial \lambda_{1}}-\sum_{j=1}^{n} c_{j}^{\prime} \frac{\partial U_{1}}{\partial y_{j}^{1}}\right)=0\left(\frac{\|\bar{v}\|}{\lambda^{\frac{3}{2}}}\right)
$$

Lastly

$$
\begin{aligned}
& \int_{\Omega} U_{1}^{p-1}\left(\bar{v}-v_{2}^{\prime}\right) v_{2}^{\prime} \\
& =\int_{\Omega} U_{1}^{p-1}\left(\bar{v}-v_{2}+a^{\prime} U_{1}+b^{\prime} \frac{\partial U_{1}}{\partial \lambda_{1}}+\sum_{j=1}^{n} c_{j}^{\prime} \frac{\partial U_{1}}{\partial y_{j}^{1}}\right)\left(v_{2}-a^{\prime} U_{1}-b^{\prime} \frac{\partial U_{1}}{\partial \lambda_{1}}-\sum_{j=1}^{n} c_{j}^{\prime} \frac{\partial U_{1}}{\partial y_{j}^{1}}\right) \\
& =\int_{\Omega} U_{1}^{p-1}\left(\left(v_{1}+v_{3}\right) v_{2}+\left(2 v_{2}-\bar{v}\right)\left(a^{\prime} U_{1}+b^{\prime} \frac{\partial U_{1}}{\partial \lambda_{1}}+\sum_{j=1}^{n} c_{j}^{\prime} \frac{\partial U_{1}}{\partial y_{j}^{1}}\right)\right. \\
& \left.\quad-\left(a^{\prime} U_{1}+b^{\prime} \frac{\partial U_{1}}{\partial \lambda_{1}}+\sum_{j=1}^{n} c_{j}^{\prime} \frac{\partial U}{\partial y_{j}^{1}}\right)^{2}\right)
\end{aligned}
$$

On one hand, using (C.21) and (C.6)

$$
\int_{\Omega} U_{1}^{p-1} v_{3} v_{2}=0\left(\int_{\substack{\Omega \mid \geq \frac{1}{4 T} \\ \Omega}} U_{1}^{p-1} \bar{v}^{2}\right)=0\left(\frac{T^{4}}{\lambda^{2}} \int_{\Omega} \bar{v}^{2}\right)=0\left(\frac{T^{4}}{\lambda^{3}}\|\bar{v}\|^{2}\right) .
$$


On the other hand, using (C.21-22,25)

$$
\begin{aligned}
& \int_{\Omega} U_{1}^{p-1} v_{1} v_{2} \\
& =\int_{B^{+}} \lambda_{1}^{2}\left(\frac{1}{\left(1+\lambda_{1}^{2}|z|^{2}\right)^{2}}-\frac{4 \lambda_{1}^{2}\left(\sum_{i=1}^{n} f_{i} z_{i}^{2}\right) z_{n}}{\left(1+\lambda_{1}^{2}|z|^{2}\right)^{3}}+0\left(\frac{\lambda_{1}^{2}|z|^{4}}{\left(1+\lambda_{1}^{2}|z|^{2}\right)^{3}}\right)\right) \\
& \quad \cdot \frac{1}{4} \tilde{\zeta}_{T}^{2}(z)(\tilde{\bar{v}}(z)-\tilde{\bar{v}}(\bar{z})) \\
& =0\left(\lambda_{1}^{4} \int_{\substack{z \in B^{+} \\
|z| \leq \frac{1}{T}}} \frac{|z|^{4}}{\left(1+\lambda_{1}^{2}|z|^{2}\right)^{3}}|\tilde{\bar{v}}(z)| d z\right) \\
& =0\left(\lambda_{1}^{4}\left(\int_{|z| \leq \frac{1}{T}} \frac{|z|^{8}}{\left(1+\lambda_{1}^{2}|z|^{2}\right)^{6}}\right)^{\frac{1}{2}}\left(\int_{B^{+}} \widetilde{\widetilde{v}}^{2}(z) d z\right)^{\frac{1}{2}}\right) \\
& =0\left(\frac{\|\bar{v}\|}{\lambda_{1}^{\frac{5}{2}} T^{\frac{n-4}{2}}}\right)
\end{aligned}
$$

where we used again oddness properties with respect to $z_{1}$, (C.6) and (D.65). It is easy to see, using (C.21), (D.17), and noticing that $\frac{\partial U_{1}}{\partial \lambda_{1}}=0\left(\frac{U_{1}}{\lambda_{1}}\right), \frac{\partial U_{1}}{y_{j}^{1}}=0\left(\lambda_{1} u_{1}\right)$, that

$$
\begin{aligned}
& \int_{\Omega} U_{1}^{p-1}\left(2 v_{2}-\bar{v}\right)\left(a^{\prime} U_{1}+b^{\prime} \frac{\partial U_{1}}{\partial \lambda_{1}}+\sum_{j=1}^{n} c_{j}^{\prime} \frac{\partial U_{1}}{\partial y_{j}^{1}}\right) \\
& =0\left[\|\bar{v}\|\left(\left|a^{\prime}\right|+\frac{\left|b^{\prime}\right|}{\lambda_{1}}+\lambda_{1} \sum_{j=1}^{n}\left|c_{j}^{\prime}\right|\right)\right] \\
& =0\left[\left(\frac{(\log \lambda)^{1 / 2}}{\lambda^{2}} \text { if } n=6 ; \frac{1}{\lambda^{2}} \text { if } n \geq 7\right)\|\bar{v}\|^{2}\right]
\end{aligned}
$$

because of (C.52). It follows also from (D.17) and the previous remark that

$$
\begin{aligned}
& \int_{\Omega} U_{1}^{p-1}\left(a^{\prime} U_{1}+b^{\prime} \frac{\partial U_{1}}{\partial \lambda_{1}}+\sum_{j=1}^{n} c_{j}^{\prime} \frac{\partial U_{1}}{\partial y_{j}^{1}}\right)^{2} \\
& =0\left(a^{\prime 2}+\frac{b^{\prime 2}}{\lambda_{1}^{2}}+\lambda_{1}^{2} \sum_{j=1}^{n} c_{j}^{\prime 2}\right) \\
& =0\left[\left(\frac{\log \lambda}{\lambda^{4}} \text { if } n=6 ; \frac{1}{\lambda^{4}} \text { if } n \geq 7\right)\|\bar{v}\|^{2}\right] \quad \text { using again (C.52). }
\end{aligned}
$$

From (C 65-66) and these last estimates, taking account of (C.19) and (3.24), we conclude

$$
\int_{\Omega}\left|\varphi_{\alpha, \lambda, y}+\bar{v}\right|^{p-1}\left(\varphi_{\alpha, \lambda, y}+\bar{v}\right) v_{2}^{\prime}=p \alpha_{1}^{p-1} \int_{\Omega} U_{1}^{p-1} v_{2}^{\prime 2}+0\left(\frac{\|\bar{v}\|}{\lambda^{\inf \left(\frac{3}{2}, \frac{n+2}{n-2}\right)}}\right) .
$$


Then, collecting (C.54-56,64,67), $\alpha_{1}$ being close to $\bar{\alpha}$ and $\sigma$ being chosen small enough, Lemma 3.1 applies and we obtain

(C.68)

$\left\|v_{2}^{\prime}\right\|^{2}=0\left[\left(\left(\frac{(\log \lambda)^{\frac{2}{3}}}{\lambda T^{2}}+\frac{T(\log \lambda)^{\frac{2}{3}}}{\lambda^{3 / 2}}\right)\right.\right.$ if $n=6 ;\left(\frac{1}{\lambda T^{2}}+\frac{T}{\lambda^{3 / 2}}+\frac{1}{\lambda^{\frac{n+2}{n-2}}}\right)$ if $\left.\left.n \geq 7\right)\|\bar{v}\|\right]$.

Then, (C.43,52) and (D.26) allow to conclude

(C.69)

$\left\|v_{2}\right\|^{2}=0\left[\left(\left(\frac{(\log \lambda)^{\frac{2}{3}}}{\lambda T^{2}}+\frac{T(\log \lambda)^{\frac{2}{3}}}{\lambda^{\frac{3}{2}}}\right)\right.\right.$ if $n=6 ;\left(\frac{1}{\lambda T^{2}}+\frac{T}{\lambda^{\frac{3}{2}}}+\frac{1}{\lambda^{\frac{n+2}{n-2}}}\right)$ if $\left.\left.n \geq 7\right)\|\bar{v}\|\right]$.

In order to make $\left\|v_{2}\right\|$ as small as possible, the best choice for $q$ in (C.19) is $q=\frac{1}{6}$. In this case (C.42,69) (3.24) lead to

$$
\left\{\begin{array}{l}
\left\|v_{4}\right\|=0\left(\frac{(\log \lambda)^{2 / 3}}{\lambda^{4 / 3}} \text { if } n=6 ; \frac{1}{\lambda^{\inf \left(\frac{4}{3}, \frac{n}{n-2}\right)}} \text { if } n \geq 7\right) \\
\left\|v_{2}\right\|=0\left(\frac{(\log \lambda)^{2 / 3}}{\lambda^{7 / 6}} \text { if } n=6 ; \frac{1}{\lambda^{\inf \left(\frac{7}{6}, \frac{n}{n-2}\right)}} \text { if } n \geq 7\right)
\end{array}\right.
$$

Estimate of $\frac{\partial K_{\mu}}{\partial y_{1}^{1}}$

We are now able to prove (C.7) - which means, with our assumptions, obtaining the suitable estimate for $\frac{\partial K_{\mu}}{\partial y_{1}^{1}}$. Since we can write, for $n \geq 6(p \leq 2)$ and $\sigma, 2-p<$ $\sigma<1$

$$
\begin{aligned}
& \left|\varphi_{\alpha, \lambda, y}+\bar{v}\right|^{p-1}\left(\varphi_{\alpha, \lambda, y}+\bar{v}\right) \\
& =\alpha_{1}^{p} U_{1}^{p}+p \alpha_{1}^{p-1} U_{1}^{p-1}\left(\sum_{j=2}^{k} \alpha_{j} U_{j}+\bar{v}\right)+\frac{p(p-1)}{2} \alpha_{1}^{p-2} U_{1}^{p-2}\left(\sum_{j=2}^{k} \alpha_{j} U_{j}+\bar{v}\right)^{2} \\
& \quad+0\left(U_{1}^{-\sigma}\left|\sum_{j=2}^{k} \alpha_{j} U_{j}+\bar{v}\right|^{p+\sigma}\right) \\
& =\alpha_{1}^{p} U_{1}^{p}+p \alpha_{1}^{p-1} U_{1}^{p-1} \bar{v}+\frac{p(p-1)}{2} \alpha_{1}^{p-2} U_{1}^{p-2} \bar{v}^{2} \\
& +0\left(U_{1}^{p-1} \sum_{j=2}^{k} U_{j}+U_{1}^{p-2} \sum_{j=2}^{k} U_{j}^{2}+U_{1}^{-\sigma} \sum_{j=2}^{k} U_{j}^{p+\sigma}+U_{1}^{-\sigma}|\bar{v}|^{p+\sigma}\right)
\end{aligned}
$$


we have

$$
\begin{aligned}
\frac{\partial K_{\mu}}{\partial y_{1}^{1}} & =\alpha_{1}^{2} \int_{\Omega} \nabla U_{1} \cdot \nabla \frac{\partial U_{1}}{\partial y_{1}^{1}}+\sum_{j=2}^{k} \alpha_{1} \alpha_{j} \int_{\Omega} \nabla U_{j} . \nabla \frac{\partial U_{1}}{\partial y_{1}^{1}}+\mu \alpha_{1}^{2} \int_{\Omega} U_{1} \frac{\partial U_{1}}{\partial y_{1}^{1}} \\
& +\mu \sum_{j=2}^{k} \alpha_{1} \alpha_{j} \int_{\Omega} U_{j} \frac{\partial U_{1}}{\partial y_{1}^{1}}-\alpha_{1}^{p} \int_{\Omega} U_{1}^{p} \frac{\partial U_{1}}{\partial y_{1}^{1}} \\
& -p \alpha_{1}^{p-1} \int_{\Omega} U_{1}^{p-1} \frac{\partial U_{1}}{\partial y_{1}^{1}} \bar{v}-\frac{p(p-1)}{2} \alpha_{1}^{p-2} \int_{\Omega} U_{1}^{p-2} \frac{\partial U_{1}}{\partial y_{1}^{1}} \bar{v}^{2} \\
& \left.=0\left[\int_{\Omega}\left(U_{1}^{p-1} \sum_{j=2}^{k} U_{j}+U_{1}^{p-2} \sum_{j=2}^{k} U_{j}^{2}+U_{1}^{-\sigma} \sum_{j=2}^{k} U_{j}^{p+\sigma}+U_{1}^{-\sigma}|\bar{v}|^{p+\sigma}\right) \mid \frac{\partial U_{1}}{\partial y_{1}^{1}}\right]\right]
\end{aligned}
$$

Estimates of Appendix D - see (D.8,19,23,26,31,43-44,46) - give

$$
\begin{aligned}
& \alpha_{1}^{2} \int_{\Omega} \nabla U_{1} \cdot \nabla \frac{\partial U_{1}}{\partial y_{1}^{1}}+\sum_{j=2}^{k} \alpha_{1} \alpha_{j} \int_{\Omega} \nabla U_{j} . \nabla \frac{\partial U_{1}}{\partial y_{1}^{1}}+\mu \alpha_{1}^{2} \int_{\Omega} U_{1} \frac{\partial U_{1}}{\partial y_{1}^{1}}+\mu \sum_{j=2}^{k} \alpha_{1} \alpha_{j} \int_{\Omega} U_{j} \frac{\partial U_{1}}{\partial y_{1}^{1}} \\
& -\alpha_{1}^{p} \int_{\Omega} U_{1}^{p} \frac{\partial U_{1}}{\partial y_{1}^{1}}+0\left[\int_{\Omega}\left(U_{1}^{p-1} \sum_{j=2}^{k} U_{j}+U_{1}^{p-2} \sum_{j=2}^{k} U_{j}^{2}+U_{1}^{-\sigma} \sum_{j=2}^{k} U_{j}^{p+\sigma}\right)\left|\frac{\partial U_{1}}{\partial y_{1}^{1}}\right|\right] \\
& =-\frac{C_{8}}{\lambda_{1}} \frac{\partial H}{\partial y_{1}^{1}}(y)+0\left(\frac{1}{\lambda^{\inf (2,(n-2)(1-\sigma))}}\right)
\end{aligned}
$$

and, noticing that $\left|\frac{\partial U_{1}}{\partial y_{1}^{1}}\right|=0\left(\lambda_{1} U_{1}\right)$, Hölder's inequality, (D.17) and (3.24) yield

$$
\begin{aligned}
\int_{\Omega} U_{1}^{-\sigma}|\bar{v}|^{p+\sigma}\left|\frac{\partial U}{\partial y_{1}^{1}}\right| & =0\left(\lambda_{1}\|\bar{v}\|^{p+\sigma}\right) \\
& =0\left(\frac{(\log \lambda)^{\frac{2}{3}(\sigma+2)}}{\lambda^{1+\sigma}} \text { if } n=6 ; \frac{1}{\lambda^{\frac{4}{n-2}+\sigma}} \text { if } n \geq 7\right) .
\end{aligned}
$$

Choosing $\sigma=\frac{n(n-4)}{(n-1)(n-2)}$, we get

$$
\begin{aligned}
\frac{\partial K_{\mu}}{\partial y_{1}^{1}} & =-\frac{C_{8}}{\lambda_{1}} \frac{\partial H}{\partial y_{1}^{1}}(y)+0\left(\frac{(\log \lambda)^{\frac{26}{5}}}{\lambda^{8 / 5}} \text { if } n=6 ; \frac{1}{\lambda^{\frac{n+2}{n-1}}} \text { if } n \geq 7\right) \\
& +\left|\int_{\Omega} U_{1}^{p-1} \frac{\partial U_{1}}{\partial y_{1}^{1}} \bar{v}\right|+\left|\int_{\Omega} U_{1}^{p-2} \frac{\partial U_{1}}{\partial y_{1}^{1}} \bar{v}^{2}\right|
\end{aligned}
$$

and it only remains to estimate the two last integrals. We have

$$
\begin{aligned}
\int_{\Omega} U_{1}^{p-1} \frac{\partial U_{1}}{\partial y_{1}^{1}} \bar{v} & =-\frac{1}{n(n+2)} \int_{\Omega} \Delta \frac{\partial U_{1}}{\partial y_{1}^{1}} \cdot \bar{v} \\
& =-\frac{1}{n(n+2)} \int_{\partial \Omega} \frac{\partial^{2} U_{1}}{\partial \nu \partial y_{1}^{1}} \cdot \bar{v} \quad \text { integrating by parts. }
\end{aligned}
$$


We note that

$$
\frac{\partial^{2} U_{1}}{\partial x_{i} \partial y_{1}^{1}}=(n-2) \lambda_{1}^{\frac{n+2}{2}}\left(\frac{\delta_{1 i}}{\left(1+\lambda_{1}^{2}|x|^{2}\right)^{\frac{n}{2}}}-\frac{n \lambda_{1}^{2} x_{1} x_{i}}{\left(1+\lambda_{1}^{2}|x|^{2}\right)^{\frac{n+2}{2}}}\right) \text {. }
$$

Therefore

$$
\frac{\partial^{2} U_{1}}{\partial \nu \partial y_{1}^{1}}=0\left(\frac{\lambda_{1}^{\frac{n+2}{2}}}{\left(1+\lambda_{1}^{2}|x|^{2}\right)^{\frac{n}{2}}}\right)
$$

and, in $\partial \Omega \cap \bar{W}$ - since, according to (C.12,13)

$$
\nu(x)=\frac{1}{\left(1+|\nabla f|^{2}\right)^{1 / 2}}\left(2 f_{1} x_{1}+0\left(\left|x^{\prime}\right|^{2}\right), \cdots, 2 f_{n-1} x_{n-1}+0\left(\left|x^{\prime}\right|^{2}\right),-1\right)
$$

we have, noticing that on $\partial \Omega, x_{n}=f\left(x^{\prime}\right)=\sum_{i=1}^{n-1} f_{i} x_{i}^{2}+0\left(\left|x^{\prime}\right|^{3}\right)$

$$
\frac{\partial^{2} U_{1}}{\partial \nu \partial y_{1}^{1}}=\frac{1}{\left(1+|\nabla f|^{2}\right)^{1 / 2}} \frac{(n-2) \lambda_{1}^{\frac{n+2}{2}}}{\left(1+\lambda_{1}^{2}|x|^{2}\right)^{n / 2}}\left(2 f_{1} x_{1}-\frac{n \lambda_{1}^{2} x_{1}}{1+\lambda_{1}^{2}|x|^{2}} \sum_{i=1}^{n-1} f_{i} x_{i}^{2}+0\left(\left|x^{\prime}\right|^{2}\right)\right)
$$

Whence, since on $\partial \Omega \cap \bar{W}$, because of (C.13-14), $|x|^{2}=\left|x^{\prime}\right|^{2}+0\left(\left|x^{\prime}\right|^{4}\right)$

$$
\frac{\partial^{2} U_{1}}{\partial \nu \partial y_{1}^{1}}=\frac{1}{\left(1+|\nabla f|^{2}\right)^{1 / 2}} \frac{(n-2) \lambda_{1}^{\frac{n+2}{2}}}{\left(1+\lambda_{1}^{2}\left|x^{\prime}\right|^{2}\right)^{n / 2}}\left(2 f_{1} x_{1}-\frac{n \lambda_{1}^{2} x_{1}}{1+\lambda_{1}^{2}\left|x^{\prime}\right|^{2}} \sum_{i=1}^{n-1} f_{i} x_{i}^{2}+0\left(\left|x^{\prime}\right|^{2}\right)\right) \text {. }
$$

Then, in view of (C.20-21), we compute

$$
\begin{aligned}
\int_{\partial \Omega} \frac{\partial^{2} U_{1}}{\partial \nu \partial y_{1}^{1}} v_{3} & =0\left[\left(\int_{\substack{x \in \partial \Omega \\
|x| \geq \frac{1}{4 T}}}\left|\frac{\partial^{2} U}{\partial \nu \partial y_{1}^{1}}\right|^{\frac{2(n-1)}{n}}\right)^{\frac{n}{2(n-1)}}\|\bar{v}\|\right] \\
& =0\left(\frac{T^{\frac{n}{2}}}{\lambda_{1}^{\frac{n}{2}}}\|\bar{v}\|\right) \quad \text { using (D.57). } \\
\int_{\partial \Omega} \frac{\partial^{2} U_{1}}{\partial \nu \partial y_{1}^{1}} v_{2}=0 & \left(\int_{\substack{x^{\prime} \in \mathbf{R}^{n-1} \\
\left|x^{\prime}\right| \leq \frac{2}{T}}} \frac{\lambda_{1}^{\frac{n+2}{2}}\left|x^{\prime}\right|}{\left(1+\lambda_{1}^{2}\left|x^{\prime}\right|^{2}\right)^{\frac{n}{2}}} v_{2}\left(x^{\prime}, f\left(x^{\prime}\right)\right) d x^{\prime}\right) \\
=0 & {\left[\lambda_{1}^{\frac{n+2}{2}}\left(\int_{\mathbf{R}^{n-1}} \frac{\left|x^{\prime}\right|^{\frac{2(n-1)}{n}} d x^{\prime}}{\left(1+\lambda_{1}^{2}\left|x^{\prime}\right|^{2}\right)^{n-1}}\right)^{\frac{n}{2(n-1)}}\left\|v_{2}\right\|\right] } \\
= & 0\left(\left\|v_{2}\right\|\right) \quad \text { using }(\mathrm{D} .63) .
\end{aligned}
$$


Lastly, (C.14,21-22,25,73) lead to

$$
\begin{aligned}
& \int_{\partial \Omega} \frac{\partial^{2} U_{1}}{\partial \nu \partial y_{1}^{1}} v_{1} \\
& =\int_{z^{\prime} \in \mathbf{R}^{n-1}} \frac{(n-2) \lambda_{1}^{\frac{n+2}{2}}}{\left(1+\lambda_{1}^{2}\left|z^{\prime}\right|^{2}\right)^{\frac{n}{2}}}\left(2 f_{1} z_{1}-\frac{n \lambda_{1}^{2} z_{1}}{1+\lambda_{1}^{2}|z|^{2}} \sum_{i=1}^{n-1} f_{i} z_{1}^{2}+0\left(\left|z^{\prime}\right|^{2}\right)\right) \cdot \tilde{\zeta}_{T}(z)\left(\widetilde{\widetilde{v}}\left(z^{\prime}, 0\right) d z\right. \\
& =0\left[\int_{\left|z^{\prime}\right| \leq \frac{1}{T}} \frac{\lambda_{1}^{\frac{n+2}{2}}\left|z^{\prime}\right|^{2}}{\left(1+\lambda_{1}^{2}|z|^{\prime 2}\right)^{\frac{n}{2}}}\left|\widetilde{\tilde{v}}\left(z^{\prime}, 0\right)\right| d z^{\prime}\right] \text { using oddness properties with respect to } z_{1} \\
& =0\left[\lambda_{1}^{\frac{n+2}{2}}\left(\int_{\mathbf{R}^{n-1}} \frac{|z|^{\frac{4(n-1)}{n}} d z^{\prime}}{\left(1+\lambda_{1}^{2}\left|z^{\prime}\right|^{2}\right)^{n-1}}\right)^{\frac{n}{2(n-1)}}\|\bar{v}\|\right] \\
& =0\left(\frac{\|\bar{v}\|}{\lambda_{1}}\right)
\end{aligned}
$$

From (C.20, 70), (3.24), $T=\lambda_{1}^{1 / 6}$, and the previous estimates we conclude

$$
\int_{\Omega} U_{1}^{p-1} \frac{\partial U_{1}}{\partial y_{1}^{1}} \bar{v}=0\left(\frac{(\log \lambda)^{2 / 3}}{\lambda^{7 / 6}} \text { if } n=6 ; \frac{1}{\lambda^{\inf \left(\frac{7}{6}, \frac{n}{n-2}\right)}} \text { if } n \geq 7\right)
$$

In the same way we compute, for $1 \leq i \leq 3$

$$
\begin{array}{rlrl}
\int_{\Omega} U_{1}^{p-2} \frac{\partial U_{1}}{\partial y_{1}^{1}} v_{i} v_{3} & =0\left[\left(\int_{|x| \geq \frac{1}{4 T}}\left|U_{1}^{p-2} \frac{\partial U_{1}}{\partial y_{1}^{1}}\right|^{\frac{p+1}{p-1}}\right)^{\frac{p-1}{p+1}}\left\|v_{i}\right\|\left\|v_{3}\right\|\right] \\
& =0\left(\frac{T^{3}}{\lambda^{2}}\|\bar{v}\|^{2}\right) & \operatorname{using}(\mathrm{D} .39)
\end{array}
$$

and, for $1 \leq i \leq 2$

$$
\begin{aligned}
\int_{\Omega} U_{1}^{p-2} \frac{\partial U_{1}}{\partial y_{1}^{1}} v_{i} v_{2} & =0\left(\lambda_{1} \int_{\Omega} U_{1}^{p-1}\left|v_{i}\right|\left|v_{2}\right|\right) \\
& =0\left(\lambda_{1}\left\|v_{i}\right\|\left\|v_{2}\right\|\right) \\
& =0\left(\lambda_{1}\|\bar{v}\|\left\|v_{2}\right\|\right)
\end{aligned}
$$


Lastly, using (C.21-22,25), we find

$$
\begin{aligned}
& \int_{\Omega} U_{1}^{p-2} \frac{\partial U_{1}}{\partial y_{1}^{1}} v_{1}^{2} \\
& =\int_{\Omega} \frac{(n-2) \lambda_{1}^{4} x_{1}}{\left(1+\lambda_{1}^{2}|x|^{2}\right)^{3}} v_{1}^{2} d x \\
& =(n-2) \lambda_{1}^{4} \int_{B^{+}} \frac{z_{1}}{\left(1+\lambda_{1}^{2}|z|^{2}\right)^{3}}\left(1-\frac{2 \lambda_{1}^{2}\left(\sum_{i=1}^{n-1} f_{i} z_{i}^{2}\right) z_{n}}{3\left(1+\lambda_{1}^{2}|z|^{2}\right)}+0\left(\frac{\lambda_{1}^{2}|z|^{4}}{1+\lambda_{1}^{2}|z|^{2}}\right)\right) \\
& =0\left(\lambda_{1}^{6} \int_{B^{+}} \frac{|z|^{5}}{\left(1+\lambda_{1}^{2}|z|^{2}\right)^{4}} \widetilde{\bar{v}}^{2}(z) d z\right) \text { because of oddness properties with respect to } z_{1} \\
& =0\left(\lambda_{1}^{6}\left(\int_{\mathbf{R}^{n}} \frac{|z|^{\frac{5 n}{2}} d z}{\left(1+\lambda_{1}^{2}|z|^{2}\right)^{2 n}}\right)^{\frac{2}{n}}\|\bar{v}\|^{z}\right) \\
& =0\left(\frac{\|\bar{v}\|^{2}}{\lambda_{1}}\right) \text { using }(\mathrm{D} \cdot 62) .
\end{aligned}
$$

Therefore, taking account of (3.24) and (C.70), we obtain

$$
\int_{\Omega} U_{1}^{p-2} \frac{\partial U_{1}}{\partial y_{1}^{\prime}} \bar{v}^{2}=0\left(\frac{(\log \lambda)^{4 / 3}}{\lambda^{7 / 6}} \text { if } n=6 ; \frac{1}{\lambda^{\inf \left(\frac{7}{6}, \frac{n}{n-2}\right)}} \text { if } n \geq 7\right) .
$$

Finally, (C.71, 74-75) show that (C.7) is satisfied.

\section{ESTIMATES}

In this last appendix, we collect the estimates of the different integral quantities which occur in the paper. In order to be as clear, short, but complete as possible, we detail the computations each time that they are necessary to convince the reader of the result, and omit them each time that they are similar to previous ones or may be easily performed.

We recall that, for $y \in \partial \Omega, \lambda>0, x \in \mathbf{R}^{n}$

$$
\left\{\begin{array}{l}
U_{\lambda, y}(x)=\frac{\lambda^{\frac{n-2}{2}}}{\left(1+\lambda^{2}|x-y|^{2}\right)^{\frac{n-2}{2}}} \\
\frac{\partial U_{\lambda, y}}{\partial \lambda}(x)=\frac{n-2}{2} \lambda^{\frac{n-4}{2}} \frac{1-\lambda^{2}|x-y|^{2}}{\left(1+\lambda^{2}|x-y|^{2}\right)^{\frac{n}{2}}} \\
\frac{\partial U_{\lambda, y}}{\partial y_{j}}(x)=-\frac{\partial U_{\lambda, y}}{\partial x_{j}}(x)=(n-2) \lambda^{\frac{n+2}{2}} \frac{(x-y)_{j}}{\left(1+\lambda^{2}|x-y|^{2}\right)^{\frac{n}{2}}}
\end{array}\right.
$$


We notice that

$$
\frac{\partial U_{\lambda, y}}{\partial \lambda}=0\left(\frac{U_{\lambda, y}}{\lambda}\right) \quad \frac{\partial U_{\lambda, y}}{\partial y_{j}}=0\left(\lambda U_{\lambda, y}\right)
$$

From now on, we assume that $n \geq 5$.

For $y \in \partial \Omega, H(y)$ denotes the mean curvature of the boundary at $y$. If we assume that, up to a translation and a rotation, $y=0$ and that, for $R>0$ small enough,

$$
\Omega \cap B(0, R)=\left\{x=\left(x^{\prime}, x_{n}\right) \in \mathbf{R}^{n-1} \times \mathbf{R} / x \in B(0, R), x_{n}>f\left(x^{\prime}\right)\right\}
$$

with

$$
f\left(x^{\prime}\right)=\sum_{i=1}^{n-1} f_{i} x_{i}^{2}+\sum_{1 \leq i \leq j \leq \ell \leq n-1} g_{i j \ell} x_{i} x_{j} x_{\ell}+0\left(\left|x^{\prime}\right|^{4}\right)
$$

we have

(D.4) $\quad H(0)=\frac{2}{n-1} \sum_{i=1}^{n-1} f_{i} ; H^{\prime}(0)=\frac{2}{n-1}\left(\sum_{j=1}^{i-1} g_{j j i}+\sum_{j=i+1}^{n-1} g_{i j j}+3 g_{i i i}\right)$.

We set

$$
\sigma_{n-1}=\operatorname{mes}\left(S^{n-1}\right)=\frac{2 \pi^{n / 2}}{\Gamma\left(\frac{n}{2}\right)}
$$

where $S^{n-1}$ is the unit sphere of $\mathbf{R}^{n}$.

We begin with the three crucial estimates

$$
\int_{\Omega}\left|\nabla U_{\lambda, y}\right|^{2}=\frac{S^{n / 2}}{2(n(n-2))^{\frac{n-2}{2}}}-C_{1} \frac{H(y)}{\lambda}+0\left(\frac{1}{\lambda^{2}}\right)
$$

with

$$
\begin{gathered}
C_{1}=\frac{(n-2)^{2} \sigma_{n-2}}{4} \frac{\Gamma\left(\frac{n+3}{2}\right) \Gamma\left(\frac{n-3}{2}\right)}{\Gamma(n)} \\
\int_{\Omega} \nabla U_{\lambda, y} \cdot \nabla \frac{\partial U_{\lambda, y}}{\partial \lambda}=\frac{C_{1}}{2} \frac{H(y)}{\lambda^{2}}+0\left(\frac{1}{\lambda^{3}}\right)
\end{gathered}
$$

$$
\int_{\Omega} \nabla U_{\lambda, y} \cdot \nabla \frac{\partial U_{\lambda, y}}{\partial \tau_{i}}=-\frac{C_{1}}{2 \lambda} \frac{\partial H}{\partial \tau_{i}}(y)+0\left(\frac{\log \lambda}{\lambda^{3}} \text { if } n=5 ; \frac{1}{\lambda^{3}} \text { if } n \geq 6\right)
$$




\section{Proof.}

(D.6) is established in [1], and the computations to show (D.7) are quite similar. We do not reproduce them, since they can be easily deduced from the proof of (D.8), which is the most delicate integral to estimate. We note that, according to (D.1)

$$
\frac{\partial^{2} U_{\lambda, y}}{\partial y_{i} \partial x_{j}}=(n-2) \lambda^{\frac{n+2}{2}}\left(\frac{\delta_{i j}}{\left(1+\lambda^{2}|x-y|^{2}\right)^{\frac{n}{2}}}-\frac{n \lambda^{2}(x-y)_{i}(x-y)_{j}}{\left(1+\lambda^{2}|x-y|^{2}\right)^{\frac{n+2}{2}}}\right)
$$

In particular

$$
\frac{\partial^{2} U_{\lambda, y}}{\partial y_{i} \partial x_{j}}=0\left(\frac{\lambda^{\frac{n+2}{2}}}{\left(1+\lambda^{2}|x-y|^{2}\right)^{\frac{n}{2}}}\right) .
$$

Without loss of generality, we may assume that $y=0, \tau_{i}=y_{i}$ for any $i, 1 \leq i \leq n-1$, and (D.2-3) hold. In the following computations, $U$ denotes the function $U_{\lambda, y}$. (D.1,9) yield

$$
\int_{\Omega \backslash B_{R}} \nabla U \cdot \nabla \frac{\partial U}{\partial y_{i}}=0\left(\lambda^{n+2} \int_{\mathbf{R}^{n} \backslash B_{R}} \frac{|x| d x}{\left(+\lambda^{2}|x|^{2}\right)^{n}}\right)=0\left(\lambda \int_{\lambda R}^{+\infty} \frac{r^{n} d r}{\left(1+r^{2}\right)^{n}}\right)
$$

using the spherical coordinates and a change of scale. Therefore

$$
\int_{\Omega \backslash B_{R}} \nabla U \cdot \nabla \frac{\partial U}{\partial y_{i}}=0\left(\frac{1}{\lambda^{n-2}}\right) \text {. }
$$

On the other hand, setting

$$
B_{R}^{+}=\left\{x=\left(x^{\prime}, x_{n}\right) \in \mathbf{R}^{n-1} \times \mathbf{R} / x \in B(0, R), x_{n}>0\right\}
$$

it follows from oddness properties that

$$
\int_{B_{R}^{+}} \nabla U \cdot \nabla \frac{\partial U}{\partial y_{i}}=0
$$

As a consequence

$$
\int_{\Omega} \nabla U \cdot \nabla \frac{\partial U}{\partial y_{i}}=-\int_{\omega^{\prime}} \nabla U \cdot \nabla \frac{\partial U}{\partial y_{i}}+\int_{\omega^{\prime \prime}} \nabla U \cdot \nabla \frac{\partial U}{\partial y_{i}}+0\left(\frac{1}{\lambda^{n-2}}\right)
$$

with

$$
\left\{\begin{array}{l}
\omega^{\prime}=\left\{x=\left(x^{\prime}, x_{n}\right) \in \mathbf{R}^{n-1} \times \mathbf{R} / x \in B(0, R), f\left(x^{\prime}\right)>0 \text { and } 0<x_{n}<f\left(x^{\prime}\right)\right\} \\
\omega^{\prime \prime}=\left\{x \in\left(x^{\prime}, x_{n}\right) \in \mathbf{R}^{n-1} \times \mathbf{R} / x \in B(0, R), f\left(x^{\prime}\right)<0 \text { and } f\left(x^{\prime}\right)<x_{n}<0\right\} .
\end{array}\right.
$$

Let us estimate the integral on $\omega^{\prime}$. For $a>0$, we define

$$
L_{a}=\left\{x \in \mathbf{R}^{n} /\left|x_{i}\right|<a, 1 \leq i \leq n\right\} .
$$


We choose $a$ small enough, so that $L_{a} \subset B_{R}$. Proceeding as previously, it is clear that

$$
\int_{\omega^{\prime} \cap L_{a}^{c}} \nabla U \cdot \nabla \frac{\partial U}{\partial y_{i}}=0\left(\frac{1}{\lambda^{n-2}}\right) .
$$

Therefore, from (D.1,9) we deduce

$$
\int_{\omega^{\prime}} \nabla U \cdot \nabla \frac{\partial U}{\partial y_{i}}=\int_{\omega^{\prime} \cap L_{a}} \nabla U \cdot \nabla \frac{\partial U}{\partial y_{i}}+0\left(\frac{1}{\lambda^{n-2}}\right)
$$

hence

$$
\begin{aligned}
\int_{\omega^{\prime}} \nabla U . \nabla \frac{\partial U}{\partial y_{i}} & =-(n-2)^{2} \lambda^{n+2} \int_{\omega^{\prime} \cap L a} \frac{x_{i} d x}{\left(1+\lambda^{2}|x|^{2}\right)^{n}} \\
& +n(n-2)^{2} \lambda^{n+4} \int_{\omega^{\prime} \cap L a} \frac{|x|^{2} x_{i} d x}{\left(1+\lambda^{2}|x|^{2}\right)^{n+1}}+0\left(\frac{1}{\lambda^{n-2}}\right)
\end{aligned}
$$

or

$$
\begin{aligned}
\int_{\omega^{\prime}} \nabla U \cdot \nabla \frac{\partial U}{\partial y_{i}} & =-(n-1)(n-2)^{2} \lambda^{n+2} \int_{\omega^{\prime} \cap L a} \frac{x_{i} d x}{\left(1+\lambda^{2}|x|^{2}\right)^{n}} \\
& -n(n-2)^{2} \lambda^{n+2} \int_{\omega^{\prime} \cap L a} \frac{x_{i} d x}{\left(1+\lambda^{2}|x|^{2}\right)^{n+1}}+0\left(\frac{1}{\lambda^{n-2}}\right) .
\end{aligned}
$$

The same expansion holds with $\omega^{\prime \prime}$ instead of $\omega^{\prime}$. Setting

$$
\Delta_{a}=\left\{x^{\prime}=\left(x_{1}, \cdots, x_{n-1}\right) \in \mathbf{R}^{n-1} /\left|x_{i}\right|<a, 1 \leq i \leq n-1\right\}
$$

we have

$$
\begin{aligned}
\int_{\omega^{\prime} \cap L_{a}} \frac{x_{i} d x}{\left(1+\lambda^{2}|x|^{2}\right)^{n}} & =\int_{f\left(x^{\prime}\right)>0}\left(\int_{0}^{f\left(x^{\prime}\right)} \frac{d x_{n}}{\left(1+\lambda^{2}\left(\left|x^{\prime}\right|^{2}+x_{n}^{2}\right)\right)^{n}}\right) d x^{\prime} \\
& =\frac{1}{\lambda} \int_{\substack{\left.\Delta_{a} \\
x^{\prime}\right)>0}} \frac{x_{i}}{\left(1+\lambda^{2}\left|x^{\prime}\right|^{2}\right)^{n-\frac{1}{2}}}\left(\int_{0}^{\frac{\lambda f\left(x^{\prime}\right)}{\left(1+\lambda^{2}\left|x^{\prime}\right|^{2}\right)^{1 / 2}}} \frac{d y}{\left(1+y^{2}\right)^{n}}\right) d x^{\prime}
\end{aligned}
$$

with $y=\frac{\lambda x_{n}}{\left(1+\lambda^{2}\left|x^{\prime}\right|^{2}\right)^{1 / 2}}$. We compute

$$
\begin{aligned}
\int_{0}^{\frac{\lambda f\left(x^{\prime}\right)}{\left(1+\lambda^{2}\left|x^{\prime}\right|^{2}\right)^{1 / 2}}} \frac{d y}{\left(1+y^{2}\right)^{n}} & =\frac{\lambda f\left(x^{\prime}\right)}{\left(1+\lambda^{2}\left|x^{\prime}\right|^{2}\right)^{1 / 2}}+0\left[\left(\frac{\lambda\left|f\left(x^{\prime}\right)\right|}{\left(1+\lambda^{2}\left|x^{\prime}\right|^{2}\right)^{1 / 2}}\right)^{3}\right] \\
& =\frac{\lambda}{\left(1+\lambda^{2}\left|x^{\prime}\right|^{2}\right)^{1 / 2}}\left(\sum_{j=1}^{n-1} f_{j} x_{j}^{2}+\sum_{1 \leq j \leq \ell \leq m \leq n-1} g_{j \ell m} x_{j} x_{\ell} x_{m}\right) \\
& +0\left(\frac{\lambda\left|x^{\prime}\right|^{4}}{\left(1+\lambda^{2}\left|x^{\prime}\right|^{2}\right)^{1 / 2}}\right) .
\end{aligned}
$$


It follows that

$$
\begin{aligned}
& \int_{\omega^{\prime} \cap L_{a}} \frac{x_{i} d x}{\left(1+\lambda^{2}|x|^{2}\right)^{n}} \\
& =\sum_{j=1}^{n-1} f_{j} \int_{\substack{\Delta a \\
f\left(x^{\prime}\right)>0}} \frac{x_{j}^{2} x_{i} d x^{\prime}}{\left(1+\lambda^{2}\left|x^{\prime}\right|^{2}\right)^{n}}+\sum_{1 \leq j \leq \ell \leq m \leq n-1} g_{j \ell m} \int_{\underset{f\left(x^{\prime}\right)>0}{\Delta a}} \frac{x_{i} x_{j} x_{\ell} x_{m} d x^{\prime}}{\left(1+\lambda^{2}\left|x^{\prime}\right|^{2}\right)^{n}} \\
& +0\left(\int_{f\left(x^{\prime}\right)>0} \frac{\left|x^{\prime}\right|^{5} d x^{\prime}}{\left(1+\lambda^{2}\left|x^{\prime}\right|^{2}\right)^{n}}\right) \text {. }
\end{aligned}
$$

We note that the same expression still holds for $\omega^{\prime \prime}$ intead of $\omega^{\prime}$, with opposite sign and integrals taken on $\left\{x^{\prime} \in \Delta_{a} / f\left(x^{\prime}\right)<0\right\}$ intead of $\left\{x^{\prime} \in \Delta_{a} / f\left(x^{\prime}\right)>0\right\}$. Therefore

$$
\begin{aligned}
& \int_{\omega^{\prime} \cap L_{a}} \frac{x_{i} d x}{\left(1+\lambda^{2}|x|^{2}\right)^{n}}-\int_{\omega^{\prime \prime} \cap L_{a}} \frac{x_{i} d x}{\left(1+\lambda^{2}|x|^{2}\right)^{n}} \\
& =\sum_{j=1}^{n-1} f_{j} \int_{\Delta_{a}} \frac{x_{j}^{2} x_{i} d x^{\prime}}{\left(1+\lambda^{2}\left|x^{\prime}\right|^{2}\right)^{n}}+\sum_{1 \leq j \leq \ell \leq m \leq n-1} g_{j \ell m} \int_{\Delta_{a}} \frac{x_{i} x_{j} x_{\ell} x_{m} d x^{\prime}}{\left(1+\lambda^{2}\left|x^{\prime}\right|^{2}\right)^{n}} \\
& +0\left(\int_{\Delta_{a}} \frac{\left|x^{\prime}\right|^{5} d x^{\prime}}{\left(1+\lambda^{2}\left|x^{\prime}\right|^{2}\right)^{n}}\right) .
\end{aligned}
$$

It follows from (D.15), oddness properties and

$$
\int_{\Delta_{a}} \frac{\left|x^{\prime}\right|^{5} d x^{\prime}}{\left(1+\lambda^{2}|x|^{2}\right)^{n}}=0\left(\frac{1}{\lambda^{n+4}} \int_{0}^{\lambda \sqrt{n-1} a} \frac{r^{n+3} d r}{\left(1+r^{2}\right)^{n}}\right)=0\left(\frac{1}{\lambda^{n+4}}\right)
$$

that

$$
\begin{aligned}
& \int_{\omega^{\prime} \cap L_{a}} \frac{x_{i} d x}{\left(1+\lambda^{2}|x|^{2}\right)^{n}}-\int_{\omega^{\prime \prime} \cap L_{a}} \frac{x_{i} d x}{\left(1+\lambda^{2}|x|^{2}\right)^{n}} \\
& =\left(\sum_{j=1}^{i-1} g_{j j i}+\sum_{j=i+1}^{n-1} g_{i j j}\right) \int_{\Delta_{a}} \frac{x_{1}^{2} x_{2}^{2} d x^{\prime}}{\left(1+\lambda^{2}\left|x^{\prime}\right|^{2}\right)^{n}}+g_{i i i} \int_{\Delta_{a}} \frac{x_{1}^{4} d x^{\prime}}{\left(1+\lambda^{2}\left|x^{\prime}\right|^{2}\right)^{n}}+0\left(\frac{1}{\lambda^{n+4}}\right) .
\end{aligned}
$$

In the same way, we get

$$
\begin{aligned}
& \int_{\omega^{\prime} \cap L_{a}} \frac{x_{i} d x}{\left(1+\lambda^{2}|x|^{2}\right)^{n+1}}-\int_{\omega^{\prime \prime} \cap L_{a}} \frac{x_{i} d x}{\left(1+\lambda^{2}|x|^{2}\right)^{n+1}} \\
& =\left(\sum_{j=1}^{i-1} g_{j j i}+\sum_{j=i+1}^{n-1} g_{i j j}\right) \int_{\Delta_{a}} \frac{x_{1}^{2} x_{2}^{2} d x^{\prime}}{\left(1+\lambda^{2}\left|x^{\prime}\right|^{2}\right)^{n+1}}+g_{i i i} \int_{\Delta_{a}} \frac{x_{1}^{4} d x^{\prime}}{\left(1+\lambda^{2}\left|x^{\prime}\right|^{2}\right)^{n+1}}+0\left(\frac{1}{\lambda^{n+4}}\right)
\end{aligned}
$$


so that, in view of (D.14), with a rescaling in the integrals

$(D .16)$

$$
\begin{aligned}
& -\int_{\omega^{\prime}} \nabla U \cdot \nabla \frac{\partial U}{\partial y_{i}}+\int_{\omega^{\prime \prime}} \nabla U \cdot \nabla \frac{\partial U}{\partial y_{i}} \\
& =-\frac{1}{\lambda}\left[( \sum _ { j = 1 } ^ { i - 1 } g _ { j j i } + \sum _ { j = i + 1 } ^ { n - 1 } g _ { i j j } ) \left((n-1)(n-2)^{2} \int_{\lambda_{\Delta_{a}}} \frac{x_{1}^{2} x_{2}^{2} d x^{\prime}}{\left(1+\left|x^{\prime}\right|^{2}\right)^{n}}\right.\right. \\
& \left.-n(n-2)^{2} \int_{\lambda \Delta_{a}} \frac{x_{1}^{2} x_{2}^{2} d x^{\prime}}{\left(1+\left|x^{\prime}\right|^{2}\right)^{n+1}}\right)+g_{i i i}\left((n-1)(n-2)^{2} \int_{\lambda \Delta_{a}} \frac{x_{1}^{4} d x^{\prime}}{\left(1+\left|x^{\prime}\right|^{2}\right)^{n}}\right. \\
& \left.\left.-n(n-2)^{2} \int_{\lambda \Delta_{a}} \frac{x_{1}^{4} d x^{\prime}}{\left(1+\left|x^{\prime}\right|^{2}\right)^{n+1}}\right)\right]+0\left(\frac{1}{\lambda^{2}}\right) .
\end{aligned}
$$

Integrating by parts, we have, for $\sigma=n$ or $\sigma=n+1$,

$$
\begin{aligned}
\int_{\lambda_{a}} \frac{x_{1}^{2} x_{2}^{2} d x^{\prime}}{\left(1+\left|x^{\prime}\right|^{2}\right)^{\sigma}} & =\frac{1}{2(\sigma-1)} \int_{\lambda \Delta_{a}} \frac{x_{1}^{2} d x^{\prime}}{\left(1+\left|x^{\prime}\right|^{2}\right)^{\sigma-1}} \\
& =\frac{1}{2(n-1)(\sigma-1)} \int_{\lambda_{\Delta_{a}}} \frac{\left|x^{\prime}\right|^{2} d x^{\prime}}{\left(1+\left|x^{\prime}\right|^{2}\right)^{\sigma-1}} \\
& =\frac{\sigma_{n-2}}{2(n-1)(\sigma-1)} \int_{0}^{+\infty} \frac{r^{n} d r}{\left(1+r^{2}\right)^{\sigma-1}}+0\left(\frac{1}{\lambda^{2 \sigma-n-3}}\right)
\end{aligned}
$$

and

$$
\int_{\lambda \Delta_{a}} \frac{x_{1}^{4} d x^{\prime}}{\left(1+\left|x^{\prime}\right|^{2}\right)^{\sigma}}=\frac{3}{2(\sigma-1)} \int_{\lambda \Delta_{a}} \frac{x_{1}^{2} d x^{\prime}}{\left(1+\left|x^{\prime}\right|^{2}\right)^{\sigma-1}} .
$$

Then, (D.11, 16) yield

$$
\begin{aligned}
& \int_{\Omega} \nabla U \cdot \nabla \frac{\partial U}{\partial y_{i}} \\
& =-\frac{(n-2)^{2} \sigma_{n-2}}{2(n-1) \lambda}\left(\sum_{j=1}^{i=1} g_{j j i}+\sum_{j=i+1}^{n-1} g_{i j j}+3 g_{i i i}\right)\left(\int_{0}^{+\infty} \frac{r^{n} d r}{\left(1+r^{2}\right)^{n-1}}-\int_{0}^{+\infty} \frac{r^{n} d r}{\left(1+r^{2}\right)^{n}}\right) \\
& +0\left(\frac{1}{\lambda^{2}}\right) .
\end{aligned}
$$

Therefore, using (D.4)

$$
\int_{\Omega} \nabla U . \nabla \frac{\partial U}{\partial y_{i}}=-\frac{(n-2)^{2} \sigma_{n-2}}{4 \lambda} \frac{\partial H}{\partial y_{i}}(0) \int_{0}^{+\infty} \frac{r^{n+2} d r}{\left(1+r^{2}\right)^{n}}+0\left(\frac{1}{\lambda^{2}}\right) .
$$

Since, as standard computations show

$$
\int_{0}^{+\infty} \frac{r^{n+2} d r}{\left(1+r^{2}\right)^{n}}=\frac{\Gamma\left(\frac{n+3}{2}\right) \Gamma\left(\frac{n-3}{2}\right)}{2 \Gamma(n)}
$$


(D.8) is proved.

Similarly to (D.6-8), we have

$$
\int_{\Omega} U_{\lambda, y}^{p+1}=\frac{S^{n / 2}}{2(n(n-2))^{n / 2}}-C_{4} \frac{H(y)}{\lambda}+0\left(\frac{1}{\lambda^{2}}\right)
$$

with

$$
\begin{gathered}
\int_{\Omega} U_{\lambda, y}^{p} \frac{\partial U_{\lambda, y}}{\partial \lambda}=\frac{n-2}{2 n} C_{4} \frac{H(y)}{\lambda^{2}}+0\left(\frac{1}{\lambda^{3}}\right) \\
\int_{\Omega} U_{\lambda, y}^{p} \frac{\partial U_{\lambda, y}}{\partial \tau_{i}}=-\frac{n-2}{2 n} \frac{C_{4}}{\lambda} \frac{\partial H}{\partial \tau_{i}}(y)+0\left(\frac{1}{\lambda^{2}}\right) .
\end{gathered}
$$

(D.17-19) are obtained in the same way as (D.6-8), and we omit the computations. In addition to these estimates, we remark that

$$
\int_{\substack{\Omega \\|x-y| \geq \rho}} U_{\lambda, y}^{p+1}=0\left(\int_{\lambda \rho}^{+\infty} \frac{r^{n-1} d r}{\left(1+r^{2}\right)^{n}}\right)
$$

so that, for $\frac{\lambda}{T}$ going to infinity

$$
\int_{|x-y| \geq \frac{1}{4 T}}^{\Omega} U_{\lambda, y}^{p+1}=0\left(\frac{T^{n}}{\lambda^{n}}\right) .
$$

Concerning quadratic integral estimates of $U_{\lambda, y}$, we have

$$
\int_{\Omega} U_{\lambda, y}^{2}=\frac{C_{5}}{\lambda^{2}}+0\left(\frac{\log \lambda}{\lambda^{3}} \text { if } n=5 ; \frac{1}{\lambda^{3}} \text { if } n \geq 6\right)
$$

with

$$
\begin{gathered}
C_{5}=\frac{\sigma_{n-1}}{4} \frac{\Gamma\left(\frac{n}{2}\right) \Gamma\left(\frac{n-4}{2}\right)}{\Gamma(n-2)} \\
\int_{\Omega} U_{\lambda, y} \frac{\partial U_{\lambda, y}}{\partial \lambda}=-\frac{C_{5}}{2 \lambda^{3}}+0\left(\frac{\log \lambda}{\lambda^{4}} \text { if } n=5 ; \frac{1}{\lambda^{4}} \text { if } n \geq 6\right)
\end{gathered}
$$




$$
\int_{\Omega} U_{\lambda, y} \frac{\partial U_{\lambda, y}}{\partial \tau_{i}}=0\left(\frac{\log \lambda}{\lambda^{3}} \text { if } n=5 ; \frac{1}{\lambda^{3}} \text { if } n \geq 6\right) .
$$

Proof. With the previous notations, we write

$$
\int_{\Omega} U^{2}=\int_{\Omega \backslash B_{R}} U^{2}+\int_{B_{R}^{+}} U^{2}-\int_{\omega^{\prime}} U^{2}+\int_{\omega^{\prime \prime}} U^{2} .
$$

We compute

$$
\begin{gathered}
\int_{\Omega \backslash B_{R}} U^{2}=0\left(\int_{|x| \geq R} \frac{\lambda^{n-2} d x}{\left(1+\lambda^{2}|x|^{2}\right)^{n-2}}\right)=0\left(\frac{1}{\lambda^{2}} \int_{\lambda R}^{+\infty} \frac{r^{n-1} d r}{\left(1+r^{2}\right)^{n-2}}\right)=0\left(\frac{1}{\lambda^{n-2}}\right) \\
\int_{B_{R}^{+}} U^{2}=\frac{1}{2} \int_{B_{R}} U^{2}=\frac{\sigma_{n-1}}{2} \int_{0}^{\lambda R} \frac{r^{n-1} d r}{\left(1+r^{2}\right)^{n-2}}=\frac{\sigma_{n-1}}{4} \frac{\Gamma\left(\frac{n}{2}\right) \Gamma\left(\frac{n-4}{2}\right)}{\Gamma(n-2)}+0\left(\frac{1}{\lambda^{n-2}}\right)
\end{gathered}
$$

since

$$
\int_{0}^{+\infty} \frac{r^{n-1} d r}{\left(1+r^{2}\right)^{n-2}}=\frac{\Gamma\left(\frac{n}{2}\right) \Gamma\left(\frac{n-4}{2}\right)}{2 \Gamma(n-2)} .
$$

Lastly

$$
\int_{\omega^{\prime}} U^{2}=\int_{\omega^{\prime} \cap L_{a}} U^{2}+\int_{\omega^{\prime} \cap L_{a}^{c}} U^{2}=\int_{\omega^{\prime} \cap L_{a}} U^{2}+0\left(\frac{1}{\lambda^{n-2}}\right)
$$

and

$$
\begin{aligned}
\int_{\omega^{\prime} \cap L_{a}} U^{2} & =\lambda^{n-2} \int_{\underset{f\left(x^{\prime}\right)>0}{\Delta a}}\left(\int_{0}^{f\left(x^{\prime}\right)} \frac{d x_{n}}{\left(1+\lambda^{2}\left|x^{\prime}\right|^{2}+\lambda^{2} x_{n}^{2}\right)^{n-2}}\right) d x^{\prime} \\
& =0\left(\lambda^{n-2} \int_{f\left(x^{\prime}\right)>0} \frac{\left|x^{\prime}\right|^{2} d x^{\prime}}{\left(1+\lambda^{2}\left|x^{\prime}\right|^{2}\right)^{n-2}}\right) \quad \text { using (D.3). }
\end{aligned}
$$

Proceeding in the same way to integrate $U^{2}$ on $\omega^{\prime \prime}$, we obtain

$$
\begin{aligned}
-\int_{\omega^{\prime}} U^{2}+\int_{\omega^{\prime \prime}} U^{2} & =0\left(\lambda^{n-2} \int_{\Delta_{a}} \frac{\left|x^{\prime}\right|^{2} d x^{\prime}}{\left(1+\lambda^{2}\left|x^{\prime}\right|^{2}\right)^{n-2}}\right) \\
& =0\left(\frac{1}{\lambda^{3}} \int_{0}^{\lambda a} \frac{r^{n} d r}{\left(1+r^{2}\right)^{n-2}}\right) \\
& =0\left(\frac{\log \lambda}{\lambda^{3}} \text { if } n=5 ; \frac{1}{\lambda^{3}} \text { if } n \geq 6\right)
\end{aligned}
$$

whence (D.21). The computations which lead to (D.22) are quite similar. Concerning (D.23), assuming that $\tau_{i}=y_{i}, 1 \leq i \leq n-1$, we write again

$$
\int_{\Omega} U \frac{\partial U}{\partial y_{i}}=\int_{\Omega \backslash B_{R}} U \frac{\partial U}{\partial y_{i}}+\int_{B_{R}^{+}} U \frac{\partial U}{\partial y_{i}}-\int_{\omega^{\prime}} U \frac{\partial U}{\partial y_{i}}+\int_{\omega^{\prime \prime}} U \frac{\partial U}{\partial y_{i}}
$$


and we compute, using (D.1)

$$
\begin{aligned}
& \int_{\Omega \backslash B_{R}} U \frac{\partial U}{\partial y_{i}}=0\left(\int_{|x| \geq R} \frac{\lambda^{n}|x| d x}{\left(1+\lambda^{2}|x|^{2}\right)^{n-1}}\right)=0\left(\frac{1}{\lambda} \int_{\lambda R}^{+\infty} \frac{r^{n} d r}{\left(1+r^{2}\right)^{n-1}}\right)=0\left(\frac{1}{\lambda^{n-2}}\right) \\
& \int_{B_{R}^{+}} U \frac{\partial U}{\partial y_{i}}=0 \text { because of oddness with respect of } y_{i} \\
& \int_{\omega^{\prime}} U \frac{\partial U}{\partial y_{i}}=\int_{\omega^{\prime} \cap L_{a}} U \frac{\partial U}{\partial y_{i}}+0\left(\frac{1}{\lambda^{n-2}}\right) \\
& \quad=\lambda^{n} \int_{f\left(x^{\prime}\right)>0}^{\Delta_{a}}\left(\int_{0}^{f\left(x^{\prime}\right)} \frac{d x_{n}}{\left(1+\lambda^{2}\left|x^{\prime}\right|^{2}+\lambda^{2} x_{n}^{2}\right)^{n-1}}\right) x_{i} d x^{\prime} \\
& =\lambda^{n-1} \int_{f\left(x^{\prime}\right)>0}^{\Delta_{a}}\left(\int_{0}^{\frac{\lambda f\left(x^{\prime}\right)}{\left(1+\lambda^{2}\left|x^{\prime}\right|^{2}\right)^{1 / 2}}} \frac{d y}{\left(1+y^{2}\right)^{n-1}}\right) \frac{x_{i} d x^{\prime}}{\left(1+\lambda^{2}\left|x^{\prime}\right|^{2}\right)^{n-\frac{3}{2}}} .
\end{aligned}
$$

(D.3) yields

$$
\begin{aligned}
\int_{0}^{\frac{\lambda f\left(x^{\prime}\right)}{\left(1+\lambda^{2}\left|x^{\prime}\right|^{2}\right)^{1 / 2}}} \frac{d y}{\left(1+y^{2}\right)^{n-1}} & =\frac{\lambda f\left(x^{\prime}\right)}{\left(1+\lambda^{2}\left|x^{\prime}\right|^{2}\right)^{1 / 2}}+0\left(\frac{\lambda^{3}\left|f\left(x^{\prime}\right)\right|^{3}}{\left(1+\lambda^{2}\left|x^{\prime}\right|^{2}\right)^{3 / 2}}\right) \\
& =\frac{\lambda}{\left(1+\lambda^{2}\left|x^{\prime}\right|^{2}\right)^{1 / 2}} \sum_{j=1}^{n-1} f_{j} x_{j}^{2}+0\left(\frac{\lambda\left|x^{\prime}\right|^{3}}{\left(1+\lambda^{2}\left|x^{\prime}\right|^{2}\right)^{1 / 2}}\right) .
\end{aligned}
$$

Therefore

$$
\int_{\omega^{\prime}} U \frac{\partial U}{\partial y_{i}}=\lambda^{n} \sum_{j=1}^{n-1} f_{j} \int_{\underset{f\left(x^{\prime}\right)>0}{\Delta}} \frac{x_{j}^{2} x_{i} d x^{\prime}}{\left(1+\lambda^{2}\left|x^{\prime}\right|^{2}\right)^{n-1}}+0\left(\lambda^{n} \int_{\underset{f\left(x^{\prime}\right)>0}{\Delta a}} \frac{\left|x^{\prime}\right|^{4} d x^{\prime}}{\left(1+\lambda^{2}\left|x^{\prime}\right|^{2}\right)^{n-1}}\right) .
$$

Treating in the same way the integral on $\omega^{\prime \prime}$, we obtain

$$
\begin{aligned}
-\int_{\omega^{\prime}} U \frac{\partial U}{\partial y_{i}}+\int_{\omega^{\prime \prime}} U \frac{\partial U}{\partial y_{i}} & =-\lambda^{n} \sum_{j=1}^{n-1} f_{j} \int_{\Delta a} \frac{x_{j}^{2} x_{i} d x^{\prime}}{\left(1+\lambda^{2}\left|x^{\prime}\right|^{2}\right)^{n-1}} \\
& +0\left(\lambda^{n} \int_{\Delta_{a}} \frac{\left|x^{\prime}\right|^{4} d x^{\prime}}{\left(1+\lambda^{2}\left|x^{\prime}\right|^{2}\right)^{n-1}}\right)
\end{aligned}
$$

The first integral vanishes, by oddness of the integrated function, and

$$
\begin{aligned}
\lambda^{n} \int_{\Delta_{a}} \frac{\left|x^{\prime}\right|^{4} d x^{\prime}}{\left(1+\lambda^{2}|x|^{2}\right)^{n-1}} & =0\left(\frac{1}{\lambda^{3}} \int_{0}^{\lambda \sqrt{n-1} a} \frac{r^{n+2} d r}{\left(1+r^{2}\right)^{n-1}}\right) \\
& =0\left(\frac{\log \lambda}{\lambda^{3}} \text { if } n=5 ; \frac{1}{\lambda^{3}} \text { if } n \geq 6\right) .
\end{aligned}
$$


(D.23) follows.

\section{Remark.}

We have

$$
\int_{\Omega \backslash B\left(y, \frac{1}{\lambda^{1 / 2}}\right)} U_{\lambda, y}^{2}=0\left(\frac{1}{\lambda^{5 / 2}}\right) \quad \text { if } n=5 .
$$

Indeed, we write

$$
\int_{\Omega \backslash B\left(y, \frac{1}{\lambda^{1 / 2}}\right)} U_{\lambda, y}^{2}=0\left(\lambda^{n-2} \int_{B(0,2 S) \backslash B\left(0, \frac{1}{\lambda^{1 / 2}}\right)} \frac{d x}{\left(1+\lambda^{2}|x|^{2}\right)^{n-2}}\right)
$$

hence, for $n=5$

$$
\int_{\Omega \backslash B\left(y, \frac{1}{\lambda^{1 / 2}}\right)} U_{\lambda, y}^{2}=0\left(\frac{1}{\lambda^{2}} \int_{\lambda^{1 / 2}}^{2 \lambda S} \frac{r^{4} d r}{\left(1+r^{2}\right)^{3}}\right)
$$

and the announced result. In addition, we remark that

$$
\int_{\substack{\Omega \\|x-y| \geq \rho}} U_{\lambda, y}^{2}=0\left(\frac{1}{\lambda^{2}} \int_{\lambda \rho}^{+\infty} \frac{r^{n-1} d r}{\left(1+r^{2}\right)^{n-2}}\right)
$$

so that, for $\frac{\lambda}{T}$ going to infinity, we have

$$
\int_{|x-y| \geq \frac{1}{4 T}}^{\Omega} U_{\lambda, y}^{2}=0\left(\frac{T^{n-4}}{\lambda^{n-2}}\right)
$$

We write now the following table, which is often used throughout the paper : 
(D.26)

$$
\left\{\begin{array}{l}
\int_{\Omega}\left|\nabla U_{i}\right|^{2}=\frac{S^{n / 2}}{2(n(n-2))^{\frac{n-2}{2}}}+0\left(\frac{1}{\lambda}\right) \\
\int_{\Omega}\left|\nabla \frac{\partial U_{i}}{\partial \lambda_{i}}\right|^{2}=\frac{C_{2}}{\lambda_{i}^{2}}+0\left(\frac{1}{\lambda^{3}}\right) \quad C_{2}=C_{2}(n)>0 \\
\int_{\Omega}\left|\nabla \frac{\partial U_{i}}{\partial y_{j}^{i}}\right|^{2}=C_{3} \lambda_{i}^{2}+0\left(\lambda_{i}\right) \quad C_{3}=C_{3}(n)>0 \\
\int_{\Omega} \nabla U_{i} \cdot \nabla \frac{\partial U_{i}}{\partial \lambda_{i}}=0\left(\frac{1}{\lambda^{2}}\right) ; \int_{\Omega} \nabla U_{i} \cdot \nabla \frac{\partial U_{i}}{\partial y_{j}^{i}}=0\left(\frac{1}{\lambda}\right) \\
\int_{\Omega} \nabla \frac{\partial U_{i}}{\partial \lambda_{i}} \cdot \nabla \frac{\partial U_{i}}{\partial y_{j}^{i}}=0\left(\frac{1}{\lambda^{2}}\right) ; \int_{\Omega} \nabla \frac{\partial U_{i}}{\partial y_{j}^{i}} \cdot \nabla \frac{\partial U_{i}}{\partial y_{\ell}^{i}}=0\left(\frac{1}{\lambda}\right) \\
\text { and, for } i \neq j \\
\int_{\Omega} \nabla U_{i} \cdot \nabla U_{j}=0\left(\frac{1}{\lambda^{n-2}}\right) ; \int_{\Omega} \nabla U_{i} \cdot \nabla \frac{\partial U_{j}}{\partial \lambda_{j}}=0\left(\frac{1}{\lambda^{n-1}}\right) ; \int_{\Omega} \nabla U_{i} \cdot \nabla \frac{\partial U_{j}}{\partial y_{i}^{j}}=0\left(\frac{1}{\lambda^{n-2}}\right) \\
\int_{\Omega} \nabla \frac{\partial U_{i}}{\partial \lambda_{i}} \cdot \nabla \frac{\partial U_{j}}{\partial \lambda_{j}}=0\left(\frac{1}{\lambda^{n}}\right) ; \int_{\Omega} \nabla \frac{\partial U_{i}}{\partial \lambda_{i}} \cdot \nabla \frac{\partial U_{j}}{\partial y_{\ell}^{j}}=0\left(\frac{1}{\lambda^{n-1}}\right) ; \int_{\Omega} \nabla \frac{\partial U_{i}}{\partial y_{\ell}^{i}} \cdot \nabla \frac{\partial U_{j}}{\partial y_{m}^{j}}=0\left(\frac{1}{\lambda^{n-2}}\right)
\end{array}\right.
$$

with, as previously, $U_{i}=U_{\lambda_{i}, y^{i}}$ and $0(f(\lambda))$ denotes a quantity dominated by $\sum_{i=1}^{k} f\left(\lambda_{i}\right)$. Setting, for $1 \leq i \leq k, 3 \leq \ell \leq n+1$

$$
\varphi_{(n+1)(i-1)+1}=U_{i} \quad \varphi_{(n+1)(i-1)+2}=\frac{\partial U_{i}}{\partial \lambda_{i}} \quad \varphi_{(n+1)(i-1)+\ell}=\frac{\partial U_{i}}{\partial \tau_{\ell}^{i}}
$$

and, for $1 \leq a, b \leq(n+1) k$

$$
m_{a, b}=\int_{\Omega} \nabla \varphi_{a} \cdot \nabla \varphi_{b}
$$

it follows from (D.26) that the matrix $M=\left(m_{a, b}\right)$ is invertible with

$$
\operatorname{det} M=C \prod_{i=1}^{k} \lambda_{i}^{2(n-2)}+0\left(\lambda^{2 k(n-2)-2}\right) \quad C=\left(\frac{S^{n / 2} C_{2} C_{3}^{n-1}}{2(n(n-2))^{\frac{n-2}{2}}}\right)^{k}
$$

The coefficients of $M^{-1}=\left(m_{a, b}^{\prime}\right)$ may be easily estimated through (D.26). Writing

$$
M^{-1}=\left(A_{i j}\right)_{1 \leq i, j \leq k}
$$


where the $A_{i j}$ 's are $(n+1) \times(n+1)$ matrices, we have

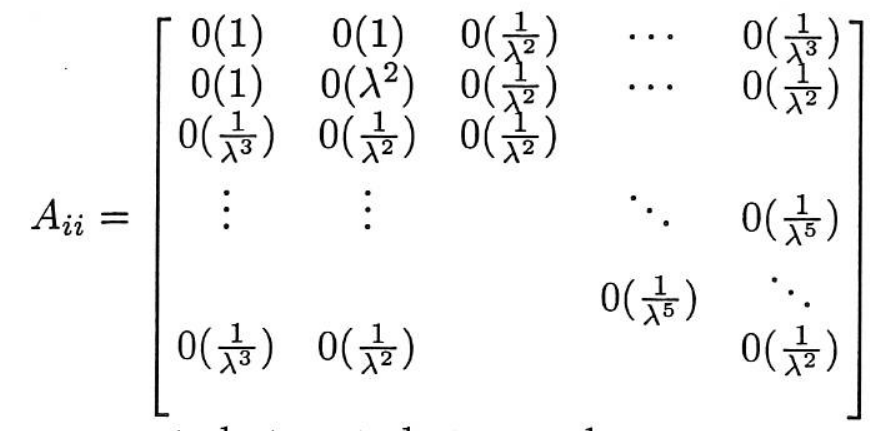

$$
\begin{aligned}
& A_{i j}=\left[\begin{array}{ccccc}
0\left(\frac{1}{\lambda^{n-2}}\right) & 0\left(\frac{1}{\lambda^{n-3}}\right) & 0\left(\frac{1}{\lambda^{n}}\right) & \cdots & 0\left(\frac{1}{\lambda^{n}}\right) \\
0\left(\frac{1}{\lambda^{n-3}}\right) & 0\left(\frac{1}{\lambda^{n-4}}\right) & 0\left(\frac{1}{\lambda^{n-1}}\right) & \cdots & \left.\frac{0(1}{\lambda^{n-1}}\right) \\
0\left(\frac{1}{\lambda^{n}}\right) & 0\left(\frac{1}{\lambda^{n-1}}\right) & & & \\
\vdots & \vdots & & 0\left(\frac{1}{\lambda^{n+2}}\right) . & \\
0\left(\frac{1}{\lambda^{n}}\right) & 0\left(\frac{1}{\lambda^{n-1}}\right) & &
\end{array}\right] i \neq j
\end{aligned}
$$

\section{Proof of (D.26)}

The estimates of the integrals over $\Omega$ of $\left|\nabla U_{i}\right|^{2}, \nabla U_{i} . \nabla \frac{\partial U_{i}}{\partial \lambda_{i}}, \nabla U_{i} . \nabla \frac{\partial U_{i}}{\partial y_{j}^{2}}$ follow from (D.6-8). The integrals of $\left|\nabla \frac{\partial U_{i}}{\partial \lambda_{i}}\right|^{2},\left|\nabla \frac{\partial U_{i}}{\partial y_{j}^{i}}\right|^{2}, \nabla \frac{\partial U_{i}}{\partial \lambda_{i}} \cdot \nabla \frac{\partial U_{i}}{\partial y_{j}^{i}}$ and $\nabla \frac{\partial U_{i}}{\partial y_{j}^{i}} \cdot \nabla \frac{\partial U_{i}}{\partial y_{\ell}^{i}}$ are computed in the same way, very easily since, in contrast with (D.6-8), we are only interested here in rough estimates.

Concerning integrals where both $U_{i}$ and $U_{j}$ occur, $i \neq j$, let us estimate the first one. We recall that $y^{i}$ and $y^{j}$ are assumed to remain far from each other, i.e. $\left|y^{i}-y^{i}\right|>d_{0}$. We write

$$
\int_{\Omega} \nabla U_{i} \cdot \nabla U_{j}=\int_{B_{i}} \nabla U_{i} \cdot \nabla U_{j}+\int_{B_{j}} \nabla U_{i} \cdot \nabla U_{j}+\int_{\Omega \backslash B_{i} \cup B_{j}} \nabla U_{i} \cdot \nabla U_{j}
$$

where $B_{i}$ and $B_{j}$ are balls centered respectively at $y^{i}$ and $y^{j}$, with radius $\frac{d_{0}}{2}$. It follows from (D.1) that

$$
\left|\nabla U_{i}\right|=0\left(\frac{1}{\lambda_{i}^{\frac{n-2}{2}}}\right) \text { on } \Omega \backslash B_{i} ;\left|\nabla U_{j}\right|=0\left(\frac{1}{\lambda_{j}^{\frac{n-2}{2}}}\right) \text { on } \Omega \backslash B_{j} .
$$

Moreover, using again (D.1), we have

$$
\int_{B_{i}}\left|\nabla U_{i}\right|=0\left(\lambda_{i}^{\frac{n+2}{2}} \int_{\mathbf{R}^{n}} \frac{|x| d x}{\left(1+\lambda_{i}^{2}|x|^{2}\right)^{\frac{n}{2}}}\right)=0\left(\frac{1}{\lambda_{i}^{\frac{n}{2}}} \int_{0}^{+\infty} \frac{r^{n} d r}{\left(1+r^{2}\right)^{\frac{n}{2}}}\right)=0\left(\frac{1}{\lambda_{i}^{\frac{n}{2}}}\right) .
$$

Therefore

$$
\int_{\Omega} \nabla U_{i} \cdot \nabla U_{j}=0\left(\frac{1}{\lambda^{n-2}}\right)
$$


The other integrals may be estimated in the same way.

\section{Remarks.}

When $y^{i}$ and $y^{j}$ are not assumed to be far from each other, and $\lambda_{i}, \lambda_{j}$ are not necessarily of the same order, it follows from computations in [6, Part 1.1], that

$$
\int_{\Omega} \nabla U_{i} \cdot \nabla U_{j}=0\left[\left(\frac{\lambda_{i}}{\lambda_{j}}+\frac{\lambda_{j}}{\lambda_{i}}+\lambda_{i} \lambda_{j}\left|y^{i}-y^{j}\right|^{2}\right)^{-\frac{n-2}{2}}\right] .
$$

In other respects, assuming that $T$ and $\frac{\lambda}{T}$ go to infinity, we have

$$
\left\{\begin{array}{l}
\int_{|x-y| \geq \frac{1}{4 T}}^{\Omega}\left|\nabla U_{\lambda, y}\right|^{2}=0\left(\left(\frac{T}{\lambda}\right)^{n-2}\right) \\
\int_{|x-y| \geq \frac{1}{4 T}}^{\Omega}\left|\nabla \frac{\partial U_{\lambda, y}}{\partial \lambda}\right|^{2}=0\left(\frac{T^{n-2}}{\lambda^{n}}\right) \\
\int_{\substack{\Omega-y \mid \geq \frac{1}{4 T} \\
\Omega}}\left|\nabla \frac{\partial U}{\partial y_{j}}\right|^{2}=0\left(\frac{T^{n}}{\lambda^{n-2}}\right)
\end{array}\right.
$$

Indeed, according to (D.1)

$$
\begin{aligned}
\int_{\substack{|x-y| \geq \frac{1}{4 T} \\
\mid}}\left|\nabla U_{\lambda, y}\right|^{2} & =0\left(\lambda^{n+2} \int_{|x| \geq \frac{1}{4 T}} \frac{|x|^{2} d x}{\left(1+\lambda^{2}|x|^{2}\right)^{n}}\right) \\
& =0\left(\int_{\frac{\lambda}{4 T}}^{+\infty} \frac{r^{n+1} d r}{\left(1+r^{2}\right)^{n}}\right) \\
& =0\left(\frac{T^{n-2}}{\lambda^{n-2}}\right)
\end{aligned}
$$

and comparable formula replacing $U_{\lambda, y}$ by $\frac{\partial U_{\lambda, y}}{\partial \lambda}$ and $\frac{\partial U_{\lambda, y}}{\partial y_{j}}$.

Finally, concerning the second derivatives of $U_{\lambda, y}$, we state

$$
\int_{\Omega}\left|\nabla \frac{\partial^{2} U_{\lambda, y}}{\partial \lambda^{2}}\right|^{2}=0\left(\frac{1}{\lambda^{4}}\right) ; \int_{\Omega}\left|\nabla \frac{\partial^{2} U_{\lambda, y}}{\partial \lambda \partial y_{j}}\right|^{2}=0(1) ; \int_{\Omega}\left|\nabla \frac{\partial^{2} U_{\lambda, y}}{\partial y_{j} \partial y_{\ell}}\right|^{2}=0\left(\lambda^{4}\right)
$$

Indeed, it follows from (D.1) that

$$
\left|\nabla \frac{\partial^{2} U_{\lambda, y}}{\partial \lambda^{2}}\right|=0\left(\frac{\lambda^{\frac{n-4}{2}}}{\left(1+\lambda^{2}|x-y|^{2}\right)^{\frac{n-1}{2}}}\right) ;\left|\nabla \frac{\partial^{2} U_{\lambda, y}}{\partial \lambda \partial y_{j}}\right|=0\left(\frac{\lambda^{\frac{n}{2}}}{\left(1+\lambda^{2}|x-y|^{2}\right)^{\frac{n}{2}}}\right)
$$

hence the expected result. 
Our last quadratic estimates are

$$
\left\{\begin{array}{l}
\int_{\Omega} U_{i}^{2}=0\left(\frac{1}{\lambda^{2}}\right) \quad \int_{\Omega}\left(\frac{\partial U_{i}}{\partial \lambda_{i}}\right)^{2}=0\left(\frac{1}{\lambda^{4}}\right) \quad \int_{\Omega}\left(\frac{\partial U_{i}}{\partial y_{j}^{i}}\right)^{2}=0(1) \\
\text { and, for } i \neq j \\
\int_{\Omega} U_{i} U_{j}=0\left(\frac{1}{\lambda^{n-2}}\right) \quad \int_{\Omega} U_{i} \frac{\partial U_{j}}{\partial \lambda_{j}}=0\left(\frac{1}{\lambda^{n-1}}\right) \quad \int_{\Omega} U_{i} \frac{\partial U_{j}}{\partial y_{\ell}^{j}}=0\left(\frac{1}{\lambda^{n-2}}\right) .
\end{array}\right.
$$

\section{Proof.}

The first estimates are a consequence of (D.21), noticing that $\frac{\partial U_{i}}{\partial \lambda_{i}}=0\left(\frac{U_{i}}{\lambda_{i}}\right)$ and $\frac{\partial U_{i}}{\partial y_{j}^{i}}=0\left(\lambda_{i} U_{i}\right)$. With the same assumptions and notations as before, we deduce from (D.1) that

$$
U_{i}=0\left(\frac{1}{\lambda_{i}^{\frac{n-2}{2}}}\right) \text { on } \Omega \backslash B_{i} \quad U_{j}=0\left(\frac{1}{\lambda_{j}^{\frac{n-2}{2}}}\right) \text { on } \Omega \backslash B_{j} \text {. }
$$

On the other hand

(D.33)

$$
\int_{B_{i}} U_{i}=0\left(\lambda_{i}^{\frac{n-2}{2}} \int_{|x| \leq \frac{d_{0}}{2}} \frac{d x}{\left(1+\lambda^{2}|x|^{2}\right)^{\frac{n-2}{2}}}\right)=0\left(\frac{1}{\lambda_{i}^{\frac{n+2}{2}}} \int_{0}^{\frac{\lambda d_{0}}{2}} \frac{r^{n-1} d r}{\left(1+r^{2}\right) \frac{n-2}{2}}\right)=0\left(\frac{1}{\lambda_{i}^{\frac{n-2}{2}}}\right)
$$

Therefore

$$
\int_{\Omega} U_{i} U_{j}=0\left(\frac{1}{\lambda^{n-2}}\right)
$$

The estimate of $\int_{\Omega} U_{i} \frac{\partial U_{j}}{\partial \lambda_{j}}$ follows from the fact that $\frac{\partial U_{j}}{\partial \lambda_{j}}=0\left(\frac{U_{j}}{\lambda_{j}}\right)$. Concerning the last integral, we remark that, according to (D.1)

$$
\left|\frac{\partial U_{j}}{\partial y_{\ell}^{j}}\right|=0\left(\frac{1}{\lambda_{j}^{\frac{n-2}{2}}}\right) \text { on } \Omega \backslash B_{j}
$$

and

(D.35)

$$
\int_{B_{j}}\left|\frac{\partial U_{j}}{\partial y_{\ell}^{j}}\right|=0\left(\lambda_{j}^{\frac{n+2}{2}} \int_{|x| \leq \frac{d_{0}}{2}} \frac{|x| d x}{\left(1+\lambda_{j}^{2}|x|^{2}\right)^{\frac{n}{2}}}\right)=0\left(\frac{1}{\lambda_{j}^{\frac{n}{2}}} \int_{0}^{\frac{\lambda_{j} d_{0}}{2}} \frac{r^{n} d r}{\left(1+r^{2}\right)^{\frac{n}{2}}}\right)=0\left(\frac{1}{\lambda_{j}^{\frac{n-2}{2}}}\right)
$$

hence the announced result.

We collect now some estimates which turn out to be very useful in the arguments of the paper. 
$(D .36) \quad\left(\int_{\Omega} U_{\lambda, y}^{\frac{p+1}{p}}\right)^{\frac{p}{p+1}}=0\left(\frac{1}{\lambda^{3 / 2}}\right.$ if $n=5 ; \frac{(\log \lambda)^{\frac{2}{3}}}{\lambda^{2}}$ if $n=6 ; \frac{1}{\lambda^{2}}$ if $\left.n \geq 7\right)$

$(D .37) \quad\left(\int_{\Omega \cap B\left(y, \frac{1}{\lambda^{1 / 2}}\right)} U_{\lambda, y}^{\frac{p+1}{p}}\right)^{\frac{p}{p+1}}=0\left(\frac{1}{\lambda^{7 / 4}}\right)$ if $n=5\left(p=\frac{7}{3}\right)$

$(D .38) \quad\left(\int_{\Omega} U_{\lambda, y}^{\frac{(p-1)(p+1)}{p}}\right)^{\frac{p}{p+1}}=\left(\frac{1}{\lambda^{3 / 2}}\right.$ if $n=5 ; \frac{(\log \lambda)^{\frac{2}{3}}}{\lambda^{2}}$ if $n=6 ; \frac{1}{\lambda^{2}}$ if $\left.n \geq 7\right)$

(D.39) $\quad\left(\int_{|x-y| \geq \frac{1}{4 T}}^{\Omega} U_{\lambda, y}^{\frac{(p-2)(p+1)}{p-1}}\left|\frac{\partial U_{\lambda, y}}{\partial y_{j}}\right|^{\frac{p-1}{p+1}}\right)^{\frac{p+1}{p-1}}=0\left(\frac{T^{3}}{\lambda^{2}}\right)$ as $T, \frac{\lambda}{T}$ go to infinity

$(D .40) \quad \int_{\Omega}\left|\frac{\partial U_{\lambda, y}}{\partial \lambda}\right|^{p+1}=0\left(\frac{1}{\lambda^{p+1}}\right) \quad \int_{\Omega}\left|\frac{\partial U_{\lambda, y}}{\partial y_{j}}\right|^{p+1}=0\left(\lambda^{p+1}\right)$

(D.41) $\quad\left(\int_{\Omega}\left|\frac{\partial U_{\lambda, y}}{\partial y_{j}}\right|^{\frac{p+1}{p}}\right)^{\frac{p}{p+1}}=0\left(\frac{1}{\lambda}\right)$.

\section{Proof.}

According to (D.1), we have

$$
\int_{\Omega} U_{\lambda, y}^{\frac{p+1}{p}}=0\left(\lambda^{\frac{n(n-2)}{n+2}} \int_{|x| \leq 2 S} \frac{d x}{\left(1+\lambda^{2}|x|^{2}\right)^{\frac{n(n-2)}{n+2}}}\right)
$$

where $S$ is a fixed number such that $\Omega \subset B(0, S)$. Thus

$$
\int_{\Omega} U_{\lambda, y}^{\frac{p+1}{p}}=0\left(\lambda^{\frac{n(n-2)}{n+2}-n} \int_{0}^{2 \lambda S} \frac{r^{n-1} d r}{\left(1+r^{2}\right)^{\frac{n(n-2)}{n+2}}}\right) .
$$

$\frac{r^{n-1}}{\left(1+r^{2}\right)^{\frac{n(n-2)}{n+2}}}$ is equivalent to $\frac{1}{r^{\frac{2 n(n-2)}{n+2}-n+1}}$ as $r$ goes to infinity, and $\frac{2 n(n-2)}{n+2}-n+1$ is larger than 1 for $n>6$, is equal to 1 for $n=6$, and is smaller than 1 for $n<6$. Therefore

$$
\int_{0}^{2 \lambda S} \frac{r^{n-1} d r}{\left(1+r^{2}\right)^{\frac{n(n-2)}{n+2}}}=0\left(\lambda^{n-\frac{2 n(n-2)}{n+2}} \text { for } n<6 ; \log \lambda \text { for } n=6 ; 1 \text { for } n>6\right)
$$

and (D.36) follows. Concerning (D.37), we have to change in the previous computation $2 \lambda S$ by $\lambda^{1 / 2}$, and we obtain

$$
\int_{0}^{\lambda^{1 / 2}} \frac{r^{n-1} d r}{\left(1+r^{2}\right)^{\frac{n(n-2)}{n+2}}}=0\left(\lambda^{\frac{1}{2}\left(n-\frac{2 n(n-2)}{n+2}\right)}\right) \text { for } n<6 .
$$


Taking $n=5$, we get the result. Next, we write $\int_{\Omega} U_{\lambda, y}^{\frac{(p-1)(p+1)}{p}}=0\left(\lambda^{\frac{4 n}{n+2}} \int_{|x| \leq 2 S} \frac{d x}{\left(1+\lambda^{2}|x|^{2}\right)^{\frac{4 n}{n+2}}}\right)=0\left(\lambda^{\frac{4 n}{n+2}-n} \int_{0}^{2 \lambda S} \frac{r^{n-1} d r}{\left(1+r^{2}\right)^{\frac{4 n}{n+2}}}\right)$. $\frac{r^{n-1}}{\left(1+r^{2}\right)^{\frac{4 n}{n+2}}}$ is equivalent to $\frac{1}{r^{\frac{8 n}{n+2}-n+1}}$ as $r$ goes to infinity, and $\frac{8 n}{n+2}-n+1$ is larger than 1 for $n<6$, is equal to 1 for $n=6$, and is smaller than 1 for $n>6$. Therefore

$$
\int_{0}^{2 \lambda S} \frac{r^{n-1} d r}{\left(1+r^{2}\right)^{\frac{4 n}{n+2}}}=0\left(1 \text { if } n=5 ; \log \lambda \text { if } n=6 ; \lambda^{n-\frac{8 n}{n+2}} \text { if } n>6\right)
$$

and (D.38) follows. We have also

$$
\begin{aligned}
\int_{\substack{\Omega \\
|x-y| \geq \frac{1}{4 T}}} U_{\lambda, y}^{\frac{(p-2)(p+1)}{p-1}}\left|\frac{\partial U_{\lambda, y}}{\partial y_{j}}\right|^{\frac{p+1}{p-1}} & =0\left(\lambda^{\frac{n(6-n)}{4}+\frac{n(n+2)}{4}} \int_{|x| \geq \frac{1}{4 T}} \frac{\cdot \cdot|x|^{\frac{n}{2}} d x}{\left(1+\lambda^{2}|x|^{2}\right)^{\frac{n(6-n)}{4}+\frac{n^{2}}{4}}}\right) \\
& =0\left(\lambda^{\frac{n}{2}} \int_{\frac{\lambda}{4 T}}^{+\infty} \frac{r^{\frac{3 n}{2}-1} d r}{\left(1+r^{2}\right)^{\frac{3 n}{2}}}\right) \\
& =0\left(\lambda^{\frac{n}{2}}\left(\frac{T}{\lambda}\right)^{\frac{3 n}{2}}\right)
\end{aligned}
$$

whence (D.39). (D.40) is a straightforward consequence of (D.17) and the fact that $\frac{\partial U_{\lambda, y}}{\partial \lambda}=0\left(\frac{U_{\lambda, y}}{\lambda}\right), \frac{\partial U_{\lambda, y}}{\partial y_{j}}=0\left(\lambda U_{x, y}\right)$. Lastly, we have

$$
\int_{\Omega}\left|\frac{\partial U_{\lambda, y}}{\partial y_{j}}\right|^{\frac{p+1}{p}}=0\left(\lambda^{n} \int_{\mathbf{R}^{n}} \frac{|x|^{\frac{2 n}{n+2}} d x}{\left(1+|x|^{2}\right)^{\frac{n^{2}}{n+2}}}\right)=0\left(\frac{1}{\lambda^{\frac{2 n}{n+2}}} \int_{0}^{+\infty} \frac{r^{\frac{2 n}{n+2}+n-1} d r}{\left(1+r^{2}\right)^{\frac{n^{2}}{n+2}}}\right)=0\left(\frac{1}{\lambda^{\frac{2 n}{n+2}}}\right)
$$

since, for every $n$, the integrals are convergent. Then, (D.41) holds.

We state some additional integral estimates of the same type, where both $U_{i}$ and $U_{j}, i \neq j$, occur. Namely

$(D .42) \quad \int_{\Omega} U_{i}^{p} U_{j}=0\left(\frac{1}{\lambda^{n-2}}\right)$

$(D .43) \quad \int_{\Omega} U_{i}^{p-1}\left|\frac{\partial U_{i}}{\partial y_{\ell}^{i}}\right| U_{j}=0\left(\frac{1}{\lambda^{n-3}}\right)$

$(D .44) \quad \int_{\Omega} U_{i}^{p-2}\left|\frac{\partial U_{i}}{\partial y_{\ell}^{2}}\right| U_{j}^{2}=0\left(\frac{1}{\lambda^{4}}\right)$

$(D .45) \quad \int_{\Omega}\left|\frac{\partial U_{i}}{\partial y_{\ell}^{2}}\right| U_{j}^{p}=0\left(\frac{1}{\lambda^{n-2}}\right)$ 
(D.46) $\quad \int_{\Omega} U_{i}^{-\sigma}\left|\frac{\partial U_{i}}{\partial y_{\ell}^{2}}\right| U_{j}^{p+\sigma}=0\left(\frac{1}{\lambda^{(n-2)(1-\sigma)}}\right) \quad$ for $n \geq 6,2-p<\sigma<1$

$(D .47) \quad\left(\int_{\Omega} \inf \left(U_{i}^{p+1}, U_{i}^{\frac{(p-2)(p+1)}{p-1}} U_{j}^{p-1}\right)\right)^{\frac{p-1}{p+1}}=0\left(\frac{1}{\lambda^{2}}\right)$.

\section{Proof.}

(D.32) yields

$$
\int_{\Omega} U_{i}^{p} U_{j}=0\left(\frac{1}{\lambda_{j}^{\frac{n-2}{2}}} \int_{B_{i}} U_{i}^{p}+\frac{1}{\lambda_{i}^{\frac{n+2}{2}}} \int_{B_{j}} U_{j}+\frac{1}{\lambda_{i}^{\frac{n+2}{2}} \lambda_{j}^{\frac{n-2}{2}}}\right)
$$

From (D.33), we know that

$$
\int_{B_{j}} U_{j}=0\left(\frac{1}{\lambda_{j}^{\frac{n-2}{2}}}\right)
$$

and

$$
\begin{aligned}
\int_{B_{i}} U_{i}^{p} & =0\left(\lambda_{i}^{\frac{n+2}{2}} \int_{|x| \leq \frac{d_{0}}{2}} \frac{d x}{\left(1+\lambda_{i}^{2}|x|^{2}\right)^{\frac{n+2}{2}}}\right) \\
& =0\left(\frac{1}{\lambda_{i}^{\frac{n-2}{2}}} \int_{0}^{\lambda_{i} \frac{d_{0}}{2}} \frac{r^{n-1} d r}{\left(1+r^{2}\right)^{\frac{n+2}{2}}}\right)
\end{aligned}
$$

so that

$(D .48)$

$$
\int_{B_{i}} U_{i}^{p}=0\left(\frac{1}{\lambda_{i}^{\frac{n-2}{2}}}\right) .
$$

This proves (D.42). (D.43) follows from (D.42) and the fact that $\left|\frac{\partial U_{i}}{\partial y_{\ell}^{2}}\right|=0\left(\lambda_{i} U_{i}\right)$. Concerning (D.44), we write

$$
\int_{\Omega} U_{i}^{p-2}\left|\frac{\partial U_{i}}{\partial y_{\ell}^{i}}\right| U_{j}^{2}=0\left(\frac{1}{\lambda_{j}^{n-2}} \int_{B_{i}} U_{i}^{p-2}\left|\frac{\partial U_{i}}{\partial y_{\ell}^{i}}\right|+\frac{1}{\lambda_{i}^{2}} \int_{B_{j}} U_{j}^{2}+\frac{1}{\lambda_{i}^{2} \lambda_{j}^{n-2}}\right)
$$

because of (D.32,34). Moreover, (D.21) ensures that

$$
\int_{B_{j}} U_{j}^{2}=0\left(\frac{1}{\lambda_{j}^{2}}\right) \text {. }
$$

Lastly

$$
\begin{aligned}
\int_{B_{i}} U_{i}^{p-2}\left|\frac{\partial U_{i}}{\partial y_{\ell}^{i}}\right| & =0\left(\lambda_{i}^{\frac{6-n}{2}+\frac{n+2}{2}} \int_{|x| \leq \frac{d_{0}}{2}} \frac{|x| d x}{\left(1+\lambda_{i}^{2}|x|^{2}\right)^{\frac{6-n}{2}+\frac{n}{2}}}\right) \\
& =0\left(\frac{1}{\lambda_{i}^{n-3}} \int_{0}^{\frac{\lambda_{i} d_{0}}{2}} \frac{r^{n} d r}{\left(1+r^{2}\right)^{3}}\right) \\
& =0\left(\frac{\log \lambda_{i}}{\lambda_{i}^{2}} \text { if } n=5 ; \frac{1}{\lambda_{i}^{2}} \text { if } n \geq 6\right)
\end{aligned}
$$


since $\frac{r^{n}}{\left(1+r^{2}\right)^{3}} \sim r^{n-6}$ as $r$ goes to infinity, and (D.44) is proved. In the same way, we write

$$
\int_{\Omega}\left|\frac{\partial U_{i}}{\partial y_{\ell}^{i}}\right| U_{j}^{p}=0\left(\frac{1}{\lambda_{j}^{\frac{n+2}{2}}} \int_{B_{i}}\left|\frac{\partial U_{i}}{\partial y_{\ell}^{i}}\right|+\frac{1}{\lambda_{i}^{\frac{n-2}{2}}} \int_{B_{j}} U_{j}^{p}+\frac{1}{\lambda_{i}^{\frac{n-2}{2}} \lambda_{j}^{\frac{n+2}{2}}}\right)=0\left(\frac{1}{\lambda^{n-2}}\right)
$$

because of (D.35, 48). We assume now that $n \geq 6$, and $2-p=\frac{n-6}{n-2}<\sigma<1$. From (D.32-34) we deduce that

$$
\begin{aligned}
\int_{\Omega} U_{i}^{-\sigma}\left|\frac{\partial U_{i}}{\partial y_{\ell}^{i}}\right| U_{j}^{p+\sigma} & =0\left(\frac{1}{\lambda_{j}^{\frac{n-2}{2}(p+\sigma)}} \int_{B_{i}} U_{i}^{-\sigma}\left|\frac{\partial U_{i}}{\partial y_{\ell}^{i}}\right|+\frac{1}{\lambda_{i}^{\frac{n-2}{2}(1-\sigma)}} \int_{B_{j}} U_{j}^{p+\sigma}\right. \\
& \left.+\frac{1}{\lambda_{i}^{\frac{n-2}{2}(1-\sigma)} \lambda_{j}^{\frac{n-2}{2}(p+\sigma)}}\right)
\end{aligned}
$$

On one hand

$$
\begin{aligned}
\int_{B_{j}} U_{j}^{p+\sigma} & =0\left(\lambda_{j}^{\frac{n-2}{2}(p+\sigma)} \int_{|x| \leq \frac{d_{0}}{2}} \frac{d x}{\left(1+\lambda_{j}^{2}|x|^{2}\right)^{\frac{n-2}{2}(p+\sigma)}}\right) \\
& =0\left(\frac{1}{\lambda_{j}^{n-\frac{n-2}{2}(p+\sigma)}} \int_{0}^{\frac{\lambda_{j} d_{0}}{2}} \frac{r^{n-1} d r}{\left(1+r^{2}\right)^{\frac{n-2}{2}(p+\sigma)}}\right)
\end{aligned}
$$

whence

$$
\int_{B_{j}} U_{j}^{p+\sigma}=0\left(\frac{1}{\lambda_{j}^{n-\frac{n-2}{2}(p+\sigma)}}\right)
$$

since the integral of $\frac{r^{n-1}}{\left(1+r^{2}\right)^{\frac{n-2}{2}(p+\sigma)}}$ is in any cases convergent. On the other hand

$$
\begin{aligned}
\int_{B_{i}} U_{i}^{-\sigma}\left|\frac{\partial U_{i}}{\partial y_{\ell}^{i}}\right| & =0\left(\lambda_{i}^{-\frac{n-2}{2} \sigma+\frac{n+2}{2}} \int_{|x| \leq \frac{d_{0}}{2}} \frac{|x| d x}{\left(1+\lambda_{i}^{2}|x|^{2}\right)^{-\frac{n-2}{2} \sigma+\frac{n}{2}}}\right) \\
& =0\left(\frac{1}{\lambda_{i}^{\frac{n}{2}+\frac{n-2}{2} \sigma}} \int_{0}^{\frac{\lambda_{i} d_{0}}{2}} \frac{r^{n} d r}{\left(1+r^{2}\right)^{\frac{n}{2}-\frac{n-2}{2} \sigma}}\right) \\
& =0\left(\frac{1}{\lambda_{i}^{\frac{n-2}{2}(1-\sigma)}}\right)
\end{aligned}
$$

since

$$
\frac{r^{n}}{\left(1+r^{2}\right)^{\frac{n}{2}-\frac{n-2}{2} \sigma}} \sim r^{(n-2) \sigma} \quad \text { as } \quad r \quad \text { goes to infinity. }
$$


Combining these results, we find (D.46). It only remains to show (D.47). We have, using (D.32)

$$
\int_{\Omega} \inf \left(U_{i}^{p+1}, U_{i}^{\frac{(p-2)(p-1)}{p-1}} U_{j}^{\frac{p+1}{p-1}}\right)=0\left(\frac{1}{\lambda_{j}^{\frac{n(n-2)}{4}}} \int_{B_{i}} U_{i}^{\frac{n(6-n)}{2(n-2)}}+\frac{1}{\lambda_{i}^{n}}\right)
$$

and

$$
\begin{aligned}
\int_{B_{i}} U_{i}^{\frac{n(6-n)}{2(n-2)}} & =\lambda_{i}^{\frac{n(6-n)}{4}} \int_{|x| \leq \frac{d_{0}}{2}} \frac{d x}{\left(1+\lambda_{i}|x|^{2}\right)^{\frac{n(6-n)}{4}}} \\
& =\frac{\sigma_{n-1}}{\lambda_{i}^{\frac{n(n-2)}{4}}} \int_{0}^{\frac{\lambda_{i} d_{0}}{4}} \frac{r^{n-1} d r}{\left(1+r^{2}\right)^{\frac{n(6-n)}{4}}} \\
& =0\left(\frac{1}{\lambda_{i}^{\frac{n(6-n)}{4}}}\right)
\end{aligned}
$$

since

$$
\frac{r^{n-1}}{\left(1+r^{2}\right)^{\frac{n(6-n)}{4}}} \sim r^{\frac{n(n-4)}{2}-1} \text { as } \quad r \text { goes to infinity. }
$$

Then

$$
\int_{\Omega} \inf \left(U_{i}^{p+1}, U_{i}^{\frac{(p-2)(p+1)}{p-1}} U_{j}^{\frac{p+1}{p-1}}\right)=0\left(\frac{1}{\lambda^{2}}\right)
$$

hence (D.47).

It only remains to estimate the boundary integrals. We have

$$
\left(\int_{\partial \Omega}\left|\frac{\partial U_{\lambda, y}}{\partial \nu}\right|^{\frac{2(n-1)}{n}}\right)^{\frac{n}{2(n-1)}}=0\left(\frac{1}{\lambda}\right)
$$

$(D .50)$

$$
\left(\int_{\partial \Omega}\left|\frac{\partial^{2} U_{\lambda, y}}{\partial \nu \partial \tau_{i}}\right|^{\frac{2(n-1)}{n}}\right)^{\frac{n}{2(n-1)}}=0(1)
$$

$(D .51)$

$$
\int_{\partial \Omega}\left|\frac{\partial U_{\lambda, y}}{\partial \nu}\right| U_{\lambda, y}=0\left(\frac{1}{\lambda}\right) ; \int_{\partial \Omega}\left|\frac{\partial U_{\lambda, y}}{\partial \nu}\right| \frac{\partial U_{\lambda, y}}{\partial \lambda}=0\left(\frac{1}{\lambda^{2}}\right) ; \int_{\partial \Omega}\left|\frac{\partial U_{\lambda, y}}{\partial \nu}\right| \frac{\partial U_{\lambda, y}}{\partial y_{i}}=0(1) .
$$




\section{Proof.}

With the nonrestrictive assumptions that $y=0$ and that $\Omega$ is defined by (D.2) in a neighbourhood of 0 , we write, for $\sigma=\left(x^{\prime}, f\left(x^{\prime}\right)\right) \in \partial \Omega$

$$
\frac{\partial U_{\lambda, y}}{\partial \nu}(\sigma)=\frac{1}{\left(1+f^{2}\left(x^{\prime}\right)\right)^{1 / 2}}\left(\sum_{i=1}^{n-1} \frac{\partial f}{\partial x_{i}} \frac{\partial U_{\lambda, y}}{\partial x_{i}}-\frac{\partial U_{\lambda, y}}{\partial x_{n}}\right)
$$

Therefore, (D.1,3) yield

$$
\frac{\partial U_{\lambda, y}}{\partial \nu}(\sigma)=-\frac{n-2}{\left(1+f^{\prime 2}\left(x^{\prime}\right)\right)^{1 / 2}} \lambda^{\frac{n+2}{2}} \frac{\sum_{i=1}^{n-1} \frac{\partial f}{\partial x_{i}} x_{i}-x_{n}}{\left(1+\lambda^{2}|x|^{2}\right)^{\frac{n}{2}}}
$$

or

$$
\frac{\partial U_{\lambda, y}}{\partial \nu}(\sigma)=-\frac{n-2}{\left(1+f^{\prime 2}\left(x^{\prime}\right)\right)^{1 / 2}} \lambda^{\frac{n+2}{2}} \frac{\sum_{i=1}^{n-1} f_{i} x_{i}^{2}+0\left(\left|x^{\prime}\right|^{3}\right)}{\left(1+\lambda^{2}|x|^{2}\right)^{\frac{n}{2}}}
$$

Since

$$
\left|\frac{d \sigma}{d x^{\prime}}\right|=\left(1+f^{\prime 2}\left(x^{\prime}\right)\right)^{1 / 2}
$$

we obtain

$$
\int_{\partial \Omega \cap B(0, R)}\left|\frac{\partial U_{\lambda, y}}{\partial \nu}\right|^{\frac{2(n-1)}{n}} d \sigma=0\left(\lambda^{\frac{(n+2)(n-1)}{n}} \int_{\left|x^{\prime}\right| \leq R} \frac{\left|x^{\prime}\right|^{\frac{4(n-1)}{n}} d x^{\prime}}{\left(1+\lambda^{2}\left|x^{\prime}\right|^{2}\right)^{n-1}}\right)
$$

using the facts that on $\partial \Omega,|x|^{2}=\left|x^{\prime}\right|^{2}+0\left(\left|x^{\prime}\right|^{4}\right)$ and $f^{\prime 2}\left(x^{\prime}\right)=0\left(\left|x^{\prime}\right|^{2}\right)$. With rescaled spherical coordinates, this yields

$$
\int_{\partial \Omega \cap B(0, R)}\left|\frac{\partial U_{\lambda, y}}{\partial \nu}\right|^{\frac{2(n-1)}{n}} d \sigma=0\left(\frac{1}{\lambda^{\frac{2(n-1)}{n}}} \int_{0}^{\lambda R^{\prime}} \frac{r^{n-2+\frac{4(n-1)}{n} d r}}{\left(1+r^{2}\right)^{n-1}}\right) .
$$

$\frac{r^{n-2+\frac{4(n-1)}{n}}}{\left(1+r^{2}\right)^{n-1}} \sim \frac{1}{r \frac{(n-2)^{2}}{n}}$ as $r$ goes to infinity, and $\frac{(n-2)^{2}}{n}>1$ for $n \geq 5$. The last integral is convergent, and

$$
\int_{\partial \Omega \cap B(0, R)}\left|\frac{\partial U_{\lambda, y}}{\partial \nu}\right|^{\frac{2(n-1)}{n}} d \sigma=0\left(\frac{1}{\lambda^{\frac{2(n-1)}{n}}}\right) .
$$

On the other hand, we deduce from (D.1) that

$$
\frac{\partial U_{\lambda, y}}{\partial \nu}=0\left(\sum_{i=1}^{n}\left|\frac{\partial U_{\lambda, y}}{\partial x_{i}}\right|\right)=0\left(\frac{\lambda^{\frac{n+2}{2}}|x|}{\left(1+\lambda^{2}|x|^{2}\right)^{n / 2}}\right)=0\left(\frac{1}{\lambda^{\frac{n-2}{2}}}\right)
$$


on $\partial \Omega \backslash B(0, R)$. Consequently

$$
\int_{\partial \Omega \backslash B(0, R)}\left|\frac{\partial U_{\lambda, y}}{\partial \nu}\right|^{\frac{2(n-1)}{n}} d \sigma=0\left(\frac{1}{\lambda^{\frac{(n-2)(n-1)}{n}}}\right)=o\left(\frac{1}{\lambda^{\frac{2(n-1)}{n}}}\right) \text { for } n \geq 5
$$

whence (D.49).

\section{Remark.}

From the previous computations - see (D.54) -, we deduce that

$$
\int_{\substack{\partial \Omega \cap B(0, R) \\|x-y| \geq \frac{1}{4 T}}}\left|\frac{\partial U_{\lambda, y}}{\partial \nu}\right|^{\frac{2(n-1)}{n}} d \sigma=0\left(\frac{1}{\lambda^{\frac{2(n-1)}{n}}} \int_{\frac{\lambda}{4 T}}^{\lambda R} \frac{r^{n-2+\frac{4(n-1)}{n}}}{\left(1+r^{2}\right)^{n-1}} d r\right)
$$

and

$$
\int_{\frac{\lambda}{4 T}}^{\lambda R} \frac{r^{n-2+\frac{4(n-1)}{n}}}{\left(1+r^{2}\right)^{n-1}} d r=0\left(\left(\frac{T}{\lambda}\right)^{\frac{(n-1)(n-4)}{n}}\right) \text { as } \quad T, \frac{\lambda}{T} \quad \text { go to infinity. }
$$

Then, we have

$$
\left(\int_{\substack{\partial \Omega \\|x-y| \geq \frac{1}{4 T}}}\left|\frac{\partial U_{\lambda, y}}{\partial \nu}\right|^{\frac{2(n-1)}{n}}\right)^{\frac{n}{2(n-1)}}=0\left(\frac{1}{\lambda}\left(\frac{T}{\lambda}\right)^{\frac{n-4}{2}}\right) .
$$

The proofs of (D.50-51) are quite similar to that of (D.49), and we omit them. We notice that, like (D.56), we have

$$
\left(\int_{\substack{\partial \Omega \\|x-y| \geq \frac{1}{4 T}}}\left|\frac{\partial^{2} U_{\lambda, y}}{\partial \nu \partial y_{i}}\right|^{\frac{2(n-1)}{n}}\right)^{\frac{n}{2(n-1)}}=0\left(\left(\frac{T}{\lambda}\right)^{\frac{n-2}{2}}\right)
$$

We end this section with the following technical estimates

$$
\begin{aligned}
& (D .58) \quad\left(\int_{|z| \leq R} \mathbf{R}^{n} \frac{|z|^{6}}{\left(1+\lambda^{2}|z|^{2}\right)^{n}} d z\right)^{1 / 2}=0\left(\frac{(\log \lambda)^{1 / 2}}{\lambda^{6}} \text { if } n=6 ; \frac{1}{\lambda^{\frac{n+6}{2}}} \text { if } n>6\right) \\
& (D .59) \quad\left(\int_{\mathbf{R}^{n}} \frac{|z|^{4}}{\left(1+\lambda^{2}|z|^{2}\right)^{n}} d z\right)^{1 / 2}=0\left(\frac{1}{\lambda^{\frac{n+4}{2}}}\right)
\end{aligned}
$$


$(D .60) \quad\left(\int_{|z| \leq \frac{1}{T}} \frac{|z|^{8}}{\left(1+\lambda^{2}|z|^{2}\right)^{n}} d z\right)^{1 / 2}=0\left(\frac{T^{\frac{n-8}{2}}}{\lambda^{n}}\right.$ if $n<8 ; \frac{\left(\log \frac{\lambda}{T}\right)}{\lambda^{8}}$ if $n=8 ; \frac{1}{\lambda^{\frac{n+8}{2}}}$ if $\left.n>8\right)$

(D.61) $\quad\left(\int_{\mathbf{R}^{n}} \frac{|z|^{8}}{\left(1+\lambda^{2}|z|^{2}\right)^{n+4}} d z\right)^{1 / 2}=0\left(\frac{1}{\lambda^{\frac{n+8}{2}}}\right)$

$(D .62) \quad\left(\int_{\mathbf{R}^{n}} \frac{|z|^{\frac{5 n}{2}}}{\left(1+\lambda^{2}|z|^{2}\right)^{2 n}} d z\right)^{\frac{2}{n}}=0\left(\frac{1}{\lambda^{7}}\right)$

$(D .63) \quad\left(\int_{\mathbf{R}^{n-1}} \frac{\left|x^{\prime}\right|^{\frac{2(n-1)}{n}}}{\left(1+\lambda^{2}\left|x^{\prime}\right|^{2}\right)^{n-1}} d x^{\prime}\right)^{\frac{n}{2(n-1)}}=0\left(\frac{1}{\lambda^{\frac{n+2}{2}}}\right)$

$(D .64) \quad\left(\int_{\mathbf{R}^{n-1}} \frac{\mid z^{\prime} \frac{4(n-1)}{n}}{\left(1+\lambda^{2}\left|z^{\prime}\right|^{2}\right)^{n-1}} d z^{\prime}\right)^{\frac{n}{2(n-1)}}=0\left(\frac{1}{\lambda^{\frac{n+4}{2}}}\right)$

$(D .65) \quad\left(\int_{|z| \leq \frac{1}{T}} \frac{|z|^{8}}{\left(1+\lambda^{2}|z|^{2}\right)^{6}} d z\right)^{\frac{1}{2}}=0\left(\frac{1}{\lambda^{6} T^{\frac{n-4}{2}}}\right)$.

All these estimates follow from straighforward computations, using spherical coordinates and a rescaling $z \mapsto \frac{z}{\lambda}, x^{\prime} \mapsto \frac{x^{\prime}}{\lambda}, z^{\prime} \mapsto \frac{z^{\prime}}{\lambda}$. 


\section{REFERENCES}

[1] Adimurthi, G. Mancini - The Neumann problem for elliptic equations with critical nonlinearity, "A tribute in honour of G. Prodi", Scuola Norm. Sup. Pisa (1991), 9-25.

[2] Adimurthi, S.L. Yadava - Existence and nonxistence of positive radial solutions of Neumann problems with critical Sobolev exponents, Arch. Rat. Mech. Anal. 115 (1991), 275-296.

[3] Adimurthi, G. Mancini, S.L. Yadava - The role of the mean curvature in semilinear Neumann problem involving critical exponent, to appear.

[4] Adimurthi, F. Pacella, S.L. Yadava - Interaction between the geometry of the boundary and positive solutions of a semilinear Neumann problem with critical nonlinearity, J. Funct. Anal. 113 (1993), 318-350.

[5] Adimurthi, F. Pacella, S.L. Yadava - Characterization of concentration points and $L^{\infty}$-estimates for solutions of semilinear Neumann problem involving the critical Sobolev exponent, to appear.

[6] A. Bahri - Critical points at infinity in some variational problems, Pitman Research Notes in Math. Series 182, Longman (1989).

[7] A. Bahri, J.M. Coron - On a nonlinear elliptic equation involving the critical Sobolev exponent : the effet of the topology of the domain, Comm. Pure Appl. Math. 41 (1988), 255-294.

[8] A. Bahri, Y. Li, O. Rey - On a variational problem with lack of compactness : the topological effet of the critical points at infinity, Calculus of Variations and Partial Diff. Equ. 3 (1995), 67-94.

[9] H. Brezis, L. Nirenberg - Positive solutions of nonlinear elliptic equations involving critical Sobolev exponents. Comm. Pure Appl. Math. XXXVI (1993), $437-477$.

[10] C. Budd, M.C. Knaap, L.A. Peletier - Asymptotic behaviour of solutions of elliptic equations with critical exponent and Neumann boundary conditions, Proc. Roy. Soc. Edinburgh 117 A (1991), 225-250.

[11] B. Gidas, J. Spruck - A priori bounds for positive solutions of nonlinear elliptic equations, Comm. Partial Diff. Equ. 6 (1981), 883-901. 
[12] E.F. Keller L.A. Segel - Initiation of slime mold aggregation viewed as an instability, J. Theoret. Biol. 26 (1970), 399-415.

[13] C.S. Lin, W.M. Ni - On the Diffusion Coefficient of a Semilinear Neumann Problem, Springer Lecture Notes 1340, Springer, New-York, Berlin, (1986).

[14] C.S. Lin, W.M. Ni, I. Takagi - Large amplitude stationary solutions to a chemotaxis system, J. Diff. Equ. 72 (1988), 1-27.

[15] P.L. Lions - The concentration compactness principle in the calculus of variations, the limit case, Rev. Mat. Iberoamericana 1.1 (1985), 145-201 and 1.2 (1985), 45-121.

[16] H. Meinhardt - Models of Biological Pattern Formation, Academic Press, London, New-York (1982).

[17] O. Rey - The role of the Green's function in a nonlinear elliptic equation involving the critical Sobolev exponent, J. Funct. Anal. 89 (1990), 1-52.

[18] O. Rey - A multiplicity result for a variational problem with lack of compactness, J. Nonlinear Anal. TMA 13 (1989), 1241-1249.

[19] O. Rey - Bifurcation from infinity in a nonlinear elliptic equation involving the limiting Sobolev exponent, Duke Math. J. 60 (1990), 815-861.

[20] X.J. Wang - Neumann problem of semilinear elliptic equations involving critical Sobolev exponents, J. Diff. Equ. 93 (1991), 283-310.

Olivier REY

URA 169 du CNRS

Centre de Mathématiques

Ecole Polytechnique

91128 - Palaiseau cedex France 